University of Tennessee Health Science Center

UTHSC Digital Commons

\title{
Genetic Variation and Sex Mediate Differential Responses to $\Delta$-9-tetrahydrocannabinol among Inbred Mice
}

Cory Parks

University of Tennessee Health Science Center

Follow this and additional works at: https://dc.uthsc.edu/dissertations

Part of the Medical Cell Biology Commons, Medical Genetics Commons, Other Chemicals and Drugs Commons, and the Other Psychiatry and Psychology Commons

\section{Recommended Citation}

Parks, Cory (https://orcid.org/0000-0003-4614-2672), "Genetic Variation and Sex Mediate Differential Responses to $\Delta$-9-tetrahydrocannabinol among Inbred Mice" (2020). Theses and Dissertations (ETD). Paper 542. http://dx.doi.org/10.21007/etd.cghs.2020.0516.

This Dissertation is brought to you for free and open access by the College of Graduate Health Sciences at UTHSC Digital Commons. It has been accepted for inclusion in Theses and Dissertations (ETD) by an authorized administrator of UTHSC Digital Commons. For more information, please contact jwelch30@uthsc.edu. 


\title{
Genetic Variation and Sex Mediate Differential Responses to $\Delta$-9-tetrahydrocannabinol among Inbred Mice
}

\begin{abstract}
The plant Cannabis sativa has been used by people for both recreational and medicinal use for thousands of years, but scientific investigation of the plant and its components didn't begin until the early nineteen hundreds when Cannabis components known as phytocannabinoids were characterized and later isolated. In the 1970's, $\Delta$-tetrahydrocannabinol (THC) was isolated and recognized as the major constituent responsible for the psychoactive and intoxicating effects associated with consumption of cannabis. This opened the door for intensive research in the field that lead to the discovery of the endogenous cannabinoid system and its associated receptors, effectors of signaling, and biosynthetic enzymes.
\end{abstract}

The primary cannabinoid receptor, cannabinoid receptor 1, is a G-protein coupled receptor (GPCR) that primarily associates with $\mathrm{Gi} / o$ proteins, giving it the properties of having mainly inhibitory actions by decreasing release of neurotransmitters and hormones. Upon receptor activation, the $\mathrm{Gi} /$ o protein disassociates with and inhibits adenylyl cyclase, decreasing cAMP production, a major second messenger of the cell. After stimulation by cannabinoids, cannabinoid receptors undergo a desensitization process where they are internalized by $\beta$-arrestins. This internalization subjects the receptors to intracellular trafficking during which the majority are degraded. This causes a decrease in surface levels of cannabinoid receptors and makes the cells less sensitive to agonists.

Cannabis is among the most widely used psychoactive drugs in the world. In the United States, use and legalization of cannabis continues to grow. The spreading use and legalization of cannabis has the social consequence of a diminished sense of risk to the individual. This can be harmful in and of itself, but cannabis THC concentrations in the U.S. have tripled over the last 20 years, giving rise to a more potent drug, potentially increasing risk of adverse effects associated with use. Effects of acute, short term use include faulty judgment and perception, memory impairment, motor skill dysfunction, alteration of mood, and low levels of attention and alertness. Effects associated with more chronic, long term use include risk of dependence, an increased risk of developing psychotic disorders such as schizophrenia, and long-term cognitive impairment.

For cannabis, and other drugs of abuse, initial response and/or tolerance to drug effects can predict later dependence and problematic use. In the work presented here, we identify sex and genetic (strain) differences in initial response and rapid tolerance to THC, the main psychoactive ingredient in cannabis, between highly genetically divergent inbred mouse strains-C57BL/6J (B6) and DBA/2J (D2). To identify variation in THC response we use the cannabinoid-induced tetrad test which quantifies the strength of agonist mediated cannabinoid receptor signaling by measuring the level of motor activity, nociception, and hypothermia elicited by receptor activation. We then extend our study of THC response variation to the BXD genetic reference population derived from B6 and D2 strains. Increasing the number of strains tested by tenfold $(\mathrm{N}=20)$ we detect significant strain and sex variation in THC response and use online tools to perform QTL mapping and correlation searches to begin to uncover potential genetic drivers of variation in response to $\mathrm{THC}$.

Document Type

Dissertation

Degree Name

Doctor of Philosophy (PhD) 


\section{Program}

Biomedical Sciences

Research Advisor

Megan K. Mulligan, PhD

\section{Keywords}

Cannabinoids, Cannabis, CB1R, QTL Mapping, THC

\section{Subject Categories}

Chemicals and Drugs | Medical Cell Biology | Medical Genetics | Medical Sciences | Medicine and Health Sciences | Other Chemicals and Drugs | Other Psychiatry and Psychology | Psychiatry and Psychology 


\section{Genetic Variation and Sex Mediate Differential Responses to $\Delta$-9-tetrahydrocannabinol among Inbred Mice}

Author:

Cory Parks
Advisor:

Megan K. Mulligan, PhD

A Dissertation Presented for The Graduate Studies Council of The University of Tennessee Health Science Center in Partial Fulfillment of the Requirements for the Doctor of Philosophy degree from

The University of Tennessee

in

Biomedical Sciences: Molecular and Translational Physiology College of Graduate Health Sciences 
Chapter 2 (C) 2020 by Mary Ann Liebert, Inc. All other material (C) 2020 by Cory Parks. All rights reserved. 


\section{DEDICATION}

I dedicate this body of work to my family: my wife, Shelby Paige Parks, who has been the best partner and mother giving me both drive and aid while progressing as a graduate student; my children, Carter Thomas Parks and Claire Olivia Brook Parks, who have given completion of this work and my degree a much deeper meaning; my parents, Alva Ray Parks and Teresa Louise Parks, for their lifelong love and encouragement that shaped me into the person I am today. 


\section{ACKNOWLEDGEMENTS}

I would like to extend my gratitude to my mentor, Dr. Megan Mulligan, for her support, trust, and guidance. The years under Dr. Mulligan provided me an exciting, dynamic, and opportunistic environment for me to develop as a scientist and accomplish the work described herein. I learned much under her wing, both in and outside of science. Thank you for being an amazing mentor and leader.

I want to acknowledge my faculty committee: Dr. Rob Williams, Dr. Bob Moore, Dr. Byron Jones, and Dr. Hao Chen. Their perspectives, critiques, and suggestions on my projects, writing, data analysis, and presentation skills has been invaluable and highly appreciated. I was lucky to have such a knowledge group at my disposal, to both help and challenge me.

I would like to thank all former and present lab members of the Mulligan group, for without the help of others this work cannot be accomplished.

I also want to acknowledge my previous undergraduate mentors, specifically Dr. Michael Taylor, who always challenged me intellectually and played a huge role in instilling my love for science, and Dr. Rebeccah Kurzhals, who through advanced lab courses and undergraduate research provided my first research and lab intensive experiences that proved crucial in preparation for grad school. 


\begin{abstract}
The plant Cannabis sativa has been used by people for both recreational and medicinal use for thousands of years, but scientific investigation of the plant and its components didn't begin until the early nineteen hundreds when Cannabis components known as phytocannabinoids were characterized and later isolated. In the 1970's, $\Delta 9$ tetrahydrocannabinol (THC) was isolated and recognized as the major constituent responsible for the psychoactive and intoxicating effects associated with consumption of cannabis. This opened the door for intensive research in the field that lead to the discovery of the endogenous cannabinoid system and its associated receptors, effectors of signaling, and biosynthetic enzymes.

The primary cannabinoid receptor, cannabinoid receptor 1, is a G-protein coupled receptor $(\mathrm{GPCR})$ that primarily associates with $\mathrm{G}_{\mathrm{i} / \mathrm{o}}$ proteins, giving it the properties of having mainly inhibitory actions by decreasing release of neurotransmitters and hormones. Upon receptor activation, the $\mathrm{G}_{\mathrm{i} / \mathrm{o}}$ protein disassociates with and inhibits adenylyl cyclase, decreasing cAMP production, a major second messenger of the cell. After stimulation by cannabinoids, cannabinoid receptors undergo a desensitization process where they are internalized by $\beta$-arrestins. This internalization subjects the receptors to intracellular trafficking during which the majority are degraded. This causes a decrease in surface levels of cannabinoid receptors and makes the cells less sensitive to agonists.
\end{abstract}

Cannabis is among the most widely used psychoactive drugs in the world. In the United States, use and legalization of cannabis continues to grow. The spreading use and legalization of cannabis has the social consequence of a diminished sense of risk to the individual. This can be harmful in and of itself, but cannabis THC concentrations in the U.S. have tripled over the last 20 years, giving rise to a more potent drug, potentially increasing risk of adverse effects associated with use. Effects of acute, short term use include faulty judgment and perception, memory impairment, motor skill dysfunction, alteration of mood, and low levels of attention and alertness. Effects associated with more chronic, long term use include risk of dependence, an increased risk of developing psychotic disorders such as schizophrenia, and long-term cognitive impairment.

For cannabis, and other drugs of abuse, initial response and/or tolerance to drug effects can predict later dependence and problematic use. In the work presented here, we identify sex and genetic (strain) differences in initial response and rapid tolerance to THC, the main psychoactive ingredient in cannabis, between highly genetically divergent inbred mouse strains - C57BL/6J (B6) and DBA/2J (D2). To identify variation in THC response we use the cannabinoid-induced tetrad test which quantifies the strength of agonist mediated cannabinoid receptor signaling by measuring the level of motor activity, nociception, and hypothermia elicited by receptor activation. We then extend our study of THC response variation to the BXD genetic reference population derived from B6 and D2 strains. Increasing the number of strains tested by tenfold $(\mathrm{N}=20)$ we detect significant strain and sex variation in THC response and use online tools to perform QTL 
mapping and correlation searches to begin to uncover potential genetic drivers of variation in response to THC. 


\section{TABLE OF CONTENTS}

CHAPTER 1. INTRODUCTION AND BACKGROUND ..................................................1

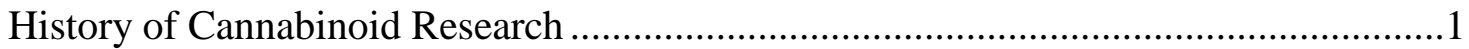

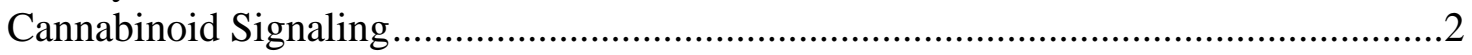

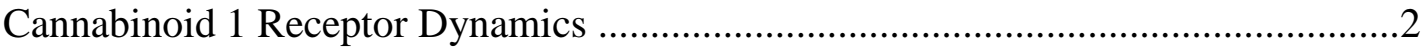

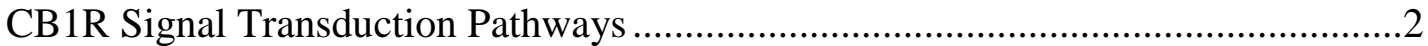

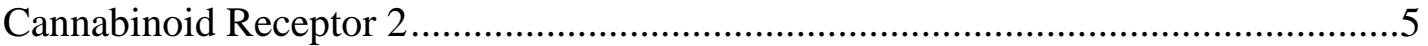

Non-Cannabinoid Receptor Activity .................................................................6

Homo- and Heterodimerization of CB1R ........................................................... 7

Distribution of Cannabinoid Receptors ................................................................ 7

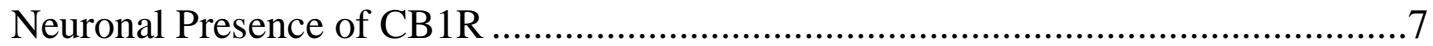

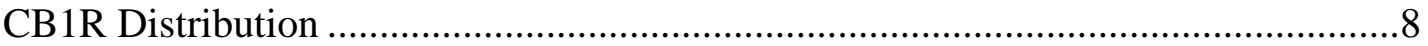

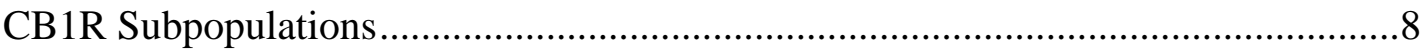

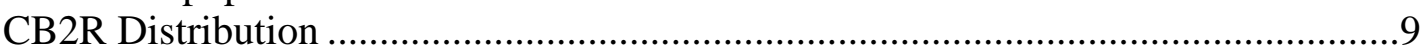

Prevalence, Use, and Effects of Cannabis ....................................................... 9

Use, Legalization, and Social Outlook...............................................................9

Physiological Effects and Pathological Roles of Cannabis and Its Receptors...........10

Behavioral Effects and Psychology of Cannabis Use .............................................. 11

Abuse and Dependence .............................................................................. 11

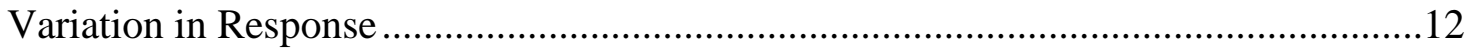

\section{CHAPTER 2. SEX AND STRAIN VARIATION IN INITIAL SENSITIVITY} AND RAPID TOLERANCE TO $\triangle 9$-TETRAHYDROCANNABINOL ....................13

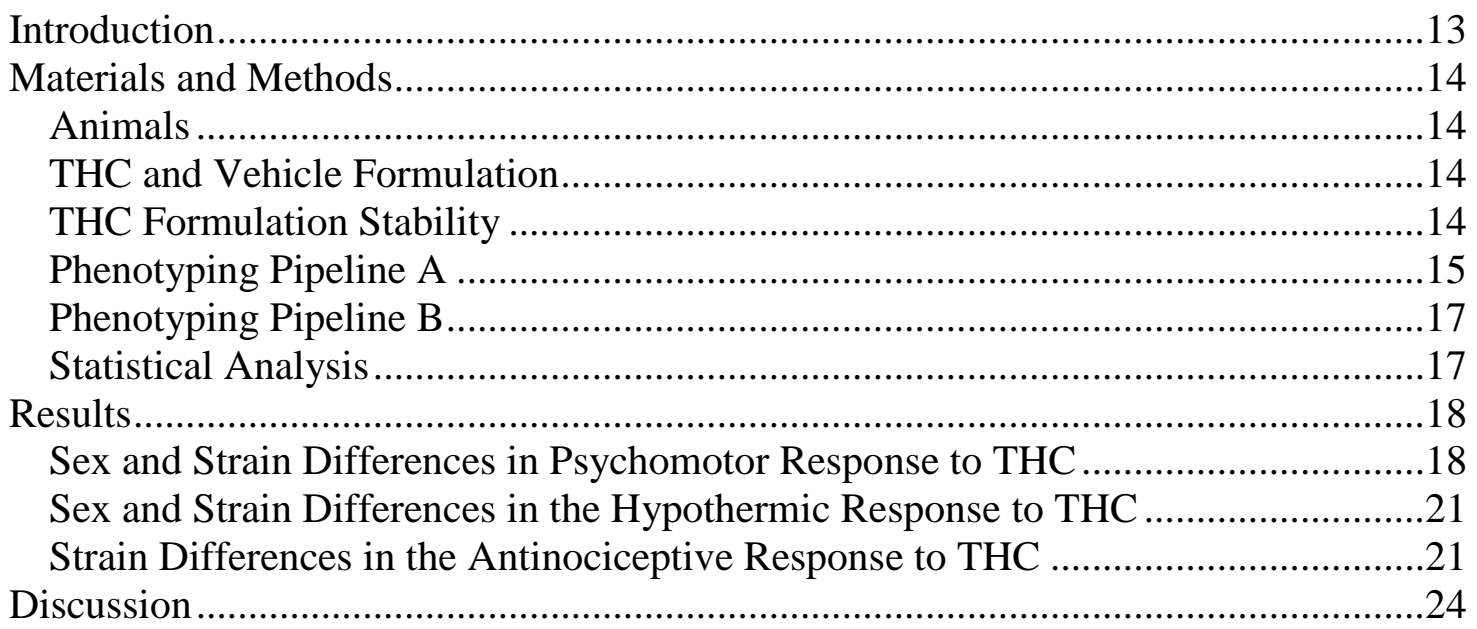

CHAPTER 3. GENETIC VARIATION IN INITIAL SENSITIVITY TO THC AMONG BXD RECOMBINANT INBRED STRAINS .................................................28

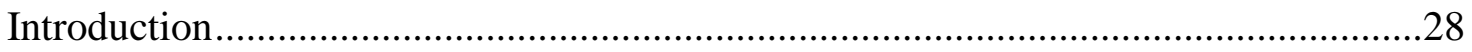

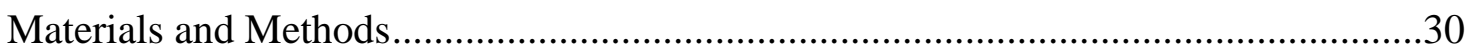

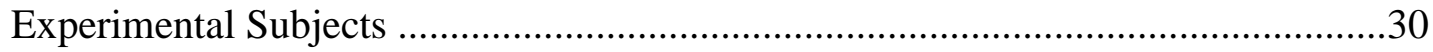

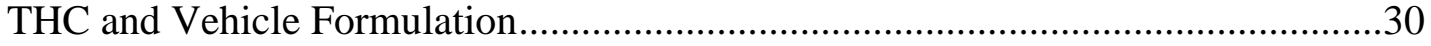

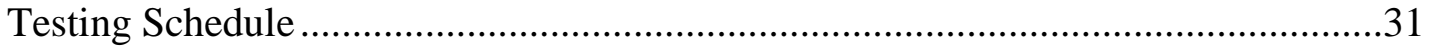


Initial Response to THC

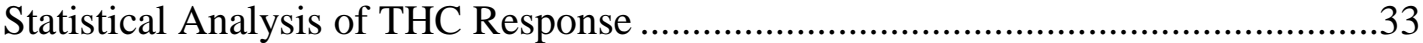

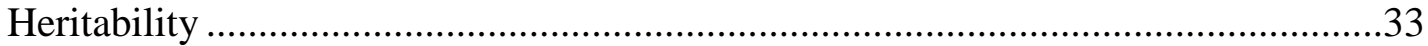

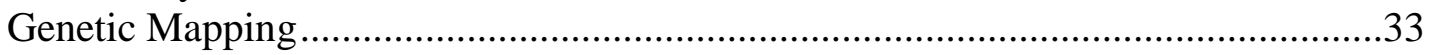

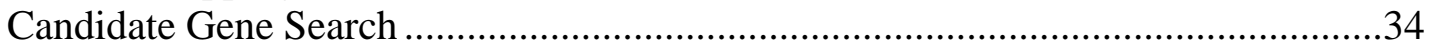

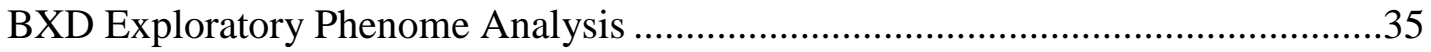

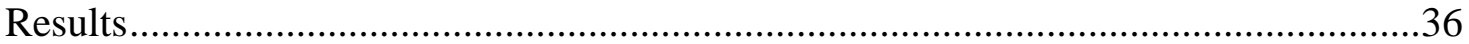

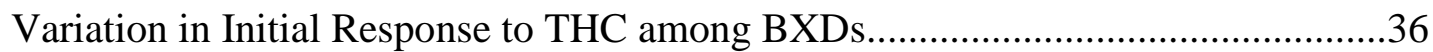

Initial Response to Mobility and Hypothermia Influenced by Chr 11 .......................39

Effectors of Endocannabinoid Signaling Identified as Positional Candidates for

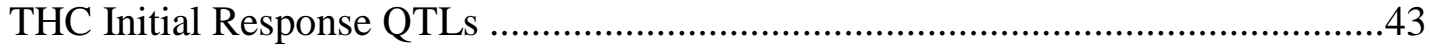

Positional Candidates Associated with Other Drugs of Abuse ...............................46

Initial Response of THC and Other Drugs Correlate .........................................47

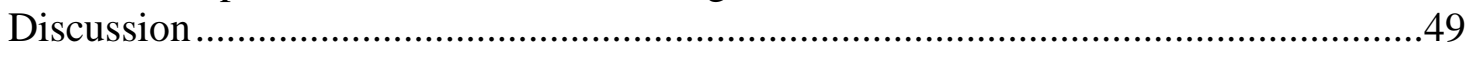

CHAPTER 4. CONCLUSIONS AND FUTURE DIRECTIONS ............................55

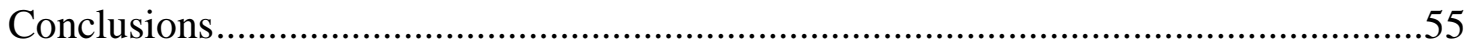

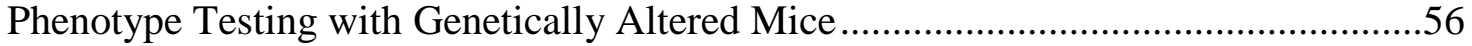

Investigation into the Pharmacogenetics of THC Response .......................................57

Distribution and Metabolism of THC ...................................................................57

CB1R Receptor Dynamics and Relevant Signaling Pathways ...............................58

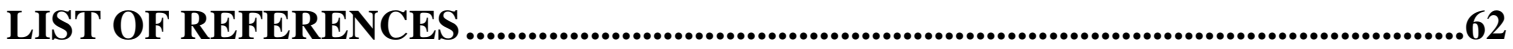

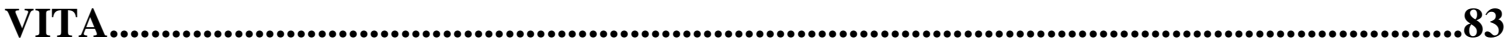




\section{LIST OF TABLES}

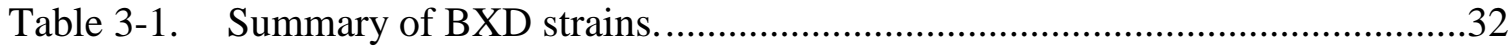

Table 3-2. Estimates of heritability among BXD and parental strains........................40

Table 3-3. Summary of suggestive QTLs for THC initial response traits. ..................42

Table 3-4. Summary of genes with high impact variants within QTL CIs. ..................44 


\section{LIST OF FIGURES}

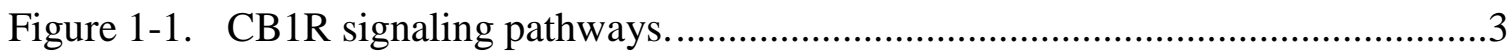

Figure 2-1. Overview of phenotype testing pipelines..................................................16

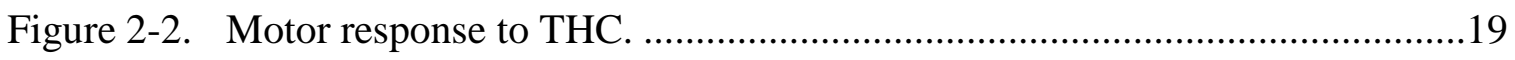

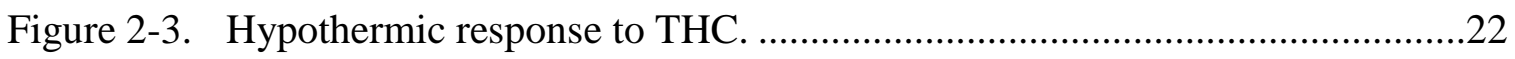

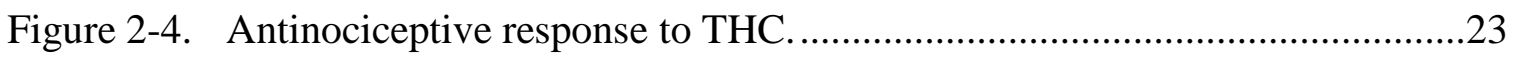

Figure 3-1. Production of recombinant inbred BXD lines. ……………………….......29

Figure 3-2. Initial response to THC in the recombinant inbred BXD strains...................37

Figure 3-3. Distribution of initial response traits...........................................................40

Figure 3-4. THC initial response trait correlations and genetic co-regulation. ................41

Figure 3-5. Summary of significant correlations between initial response to THC and BXD legacy drug response traits..........................................................48

Figure 3-6. Exercise in mapping power with number of BXD strains............................52

Figure 3-7. Power calculation of BXD family .........................................................53

Figure 4-1. Baseline CB1 receptor differences between B6 and D2..............................59

Figure 4-2. Gene expression differences in cannabinoid signaling genes across the BXD population. 


\section{LIST OF ABBREVIATIONS}

$\mathrm{AC}$

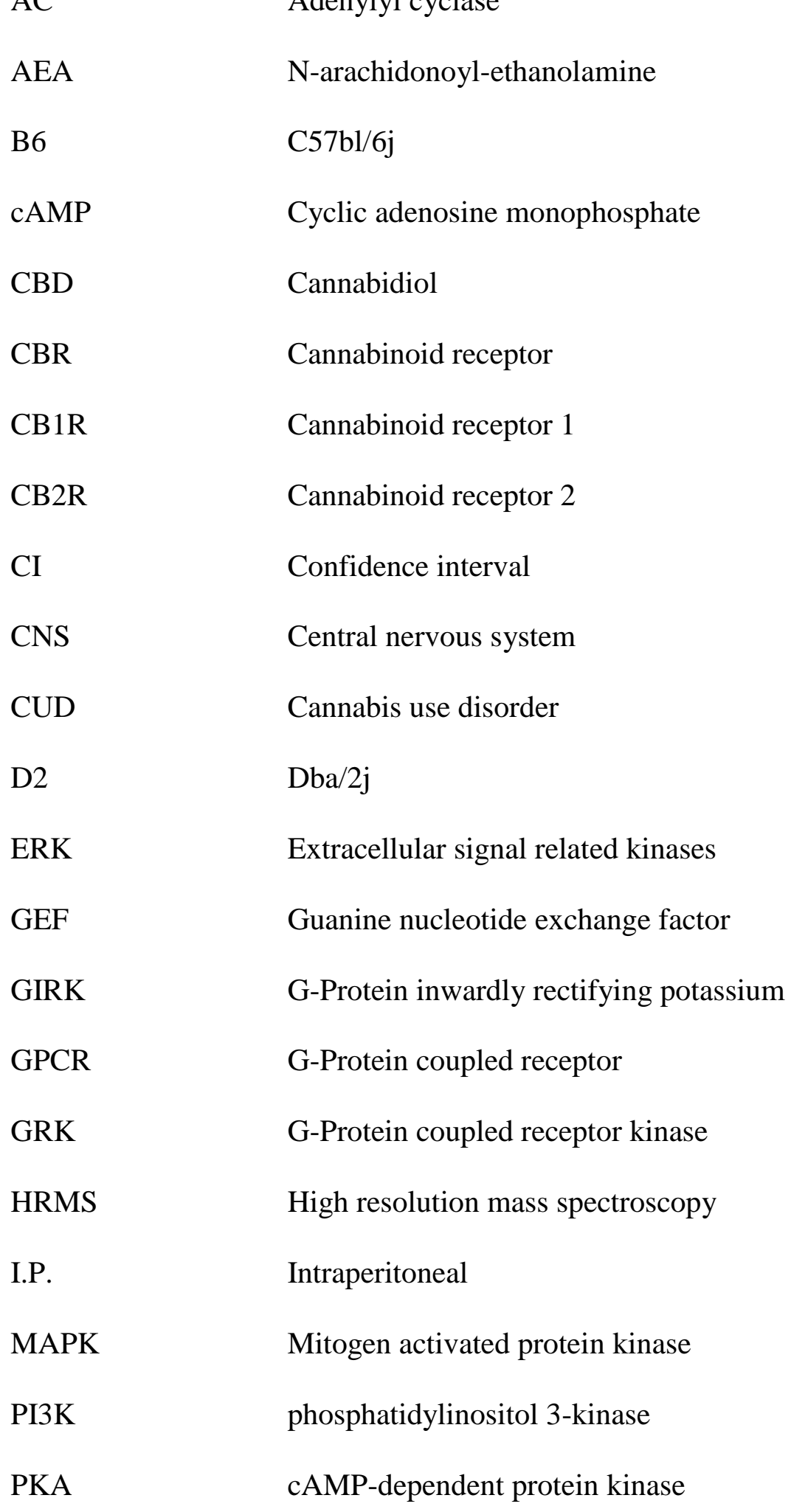

Adenylyl cyclase

$\mathrm{N}$-arachidonoyl-ethanolamine

$\mathrm{C} 57 \mathrm{bl} / 6 \mathrm{j}$

Cyclic adenosine monophosphate

Cannabidiol

Cannabinoid receptor

Cannabinoid receptor 1

Cannabinoid receptor 2

Confidence interval

Central nervous system

Cannabis use disorder 


$\begin{array}{ll}\text { PNS } & \text { Peripheral nervous system } \\ \text { PPAR } & \text { Peroxisome proliferator activated receptors } \\ \text { QTL } & \text { Quantitative trait locus } \\ \text { THC } & \Delta-9 \text {-tetrahydrocannibnol } \\ \text { TRP } & \text { Transient receptor potential } \\ \text { VEGF } & \text { vascular endothelial growth factor }\end{array}$




\section{CHAPTER 1. INTRODUCTION AND BACKGROUND}

\section{History of Cannabinoid Research}

The flowering plant Cannabis sativa, known most commonly as marijuana, is one of the first plants to be used for therapeutic and recreational use and even the occasional religious ceremony. Records of medicinal use date back as far as 5,000 years in China with therapeutic uses such as pain relief, mood stabilization, and reduced nausea documented during this time [1]. However, scientific research didn't begin until the early $20^{\text {th }}$ century when the major constituents of cannabis, phytocannabinoids, were first characterized. The first of these plant cannabinoids, Cannabinol (CBN) and Cannabidiol (CBD), were isolated approximately 80 years ago [2]. The versatility of cannabis's effects didn't begin to be unveiled until the isolation and partial synthesis of the phytocannabinoid $\Delta 9$-tetrahydrocannabinol (THC) about 30 years later [3]. From the approximate 550 total compounds currently identified in cannabis, 144 are phytocannabinoids, and THC is considered the primary psychoactive component of the plant [4].

With further identification and understanding of phytocannabinoids, the idea of the presence or absence of cannabinoid receptors (CBRs) became a debated topic in the field [2]. The discovery and synthesis of THC paved the way for the construction of high potency synthetic cannabinoids with similar structures to natural phytocannabinoids. The availability and use of these synthetic cannabinoids directed research to answering the question of the existence of endogenous CBRs. Evidence of their presence was first demonstrated by the ability of cannabinoids to inhibit cAMP formation, a Gi/Go protein pathway that is turned on through GPCR surface proteins [5]. This was followed by the successful cloning of the first CBR, cannabinoid receptor 1 (CB1R), from mammalian brain $[6,7]$, followed closely by the identification of a second CBR, eventually named cannabinoid receptor 2 (CB2R), which was cloned from immune tissue [8].

With these newly discovered CBRs, ligands that are naturally expressed in the body, $\mathrm{N}$-arachidonoyl-ethanolamine (AEA; anandamide) and 2-arachidonoylglycerol (2AG), that bind these surface proteins were isolated and named endogenous cannabinoids or endocannabinoids $[9,10]$. These two originally identified molecules remain the best studied endocannabinoids in the field. They are endogenous lipids whose precursors are present in the cell membrane and when needed can be released from the lipid bilayer, converted into endocannabinoids through enzyme reactions, and released into the extracellular space as neuromodulators [11]. The efficacy of each endogenous CBR agonist varies. 2-AG has high efficacy for both $\mathrm{CB} 1 \mathrm{R}$ and $\mathrm{CB} 2 \mathrm{R}$ while anandamide has low efficacy for CB1R and extremely low efficacy for CB2R [12, 13]. These endocannabinoids, their corresponding CBRs, and relevant enzymes comprise the endocannabinoid system. This system remains a research field of high interest in terms of further exploration of canonical, noncanonical, and adjacent signaling pathways and the potential development of these pathways for therapeutic and medical use. 


\section{Cannabinoid Signaling}

\section{Cannabinoid 1 Receptor Dynamics}

Cannabinoids have a remarkable degree of chemical diversity and consist of three classes: endocannabinoids (ex: 2-AG), phytocannabinoids (ex: THC), and synthetic cannabinoids (ex: WIN55,212-2). In the central nervous system (CNS), the primary target of each class of molecules is CB1R. Cannabinoid receptor signaling has a divergent network of intracellular pathways, (Figure 1-1) [14], having predominantly inhibitory actions by decreasing release of neurotransmitters and hormones [15-17]. CB1 receptors accomplish this through their association with Gi/o proteins to inhibit adenylyl cyclase (AC), regulate certain potassium and calcium ion channels, and activate mitogen activated protein kinase (MAPK) $[18,19]$. As with other GPCRs, activation of CBRs initiates desensitization, a process where receptor levels diminish on the cell surface due to the presence of agonist. The first step is the phosphorylation of ligand-bound receptors by a $G$ protein coupled receptor kinase (GRK). Phosphorylated receptors are then detected by arrestin proteins, specifically $\beta$-arrestin 2 for CB1R, which bind to the receptor and initiate internalization [20,21]. Once internalized, CB1R is subject to intracellular trafficking where it can either be recycled to the surface or degraded by lysosomes. Evidence exists that degradation is the primary fate for internalized CB1 receptors [22, 23].

$\mathrm{CB} 1 \mathrm{R}$ is also able to activate other $\mathrm{G}$ proteins given specific cell types and ligands present [24]. In cells with specific AC isoforms CB1R is capable of pairing with and activating G $\beta \gamma$ subunits [25]. Through various cell culture models CB1R, both transfected and endogenously expressed, can increase intracellular calcium levels through Gq/11 subunits [26]. CB1Rs ability to increase intracellular calcium concentration through $\mathrm{Gq} / 11$ has also been noted in astrocytes, causing a release of glutamate and influencing synaptic transmission of pyramidal neurons [27]. Finally, it has also been proposed that under certain conditions, such as in cultured striatal neurons with coactivation of dopamine receptor 2 and high concentrations of certain agonist, CB1R might be capable of stimulating cAMP production through association of the Gs protein [28-30]. This activity remains unclear due to the inability of researchers to isolate CB1R bound $\mathrm{G}$ proteins other than Gi/o [31] and recent evidence suggests that CB1 receptormediated increases in cAMP are due to reduced Gi/o function rather than Gs activation [32].

\section{CB1R Signal Transduction Pathways}

Arguably the most meaningful signaling effect of CB1R induction is the inhibition of AC, an enzyme responsible for the production of intracellular cyclic adenosine 3,5-monophoshate (cAMP). The first second messenger to be discovered [33] and the first signal transduction pathway characterized through cannabinoid agonist activity [34], cAMP plays an essential role in the cellular response to numerous 


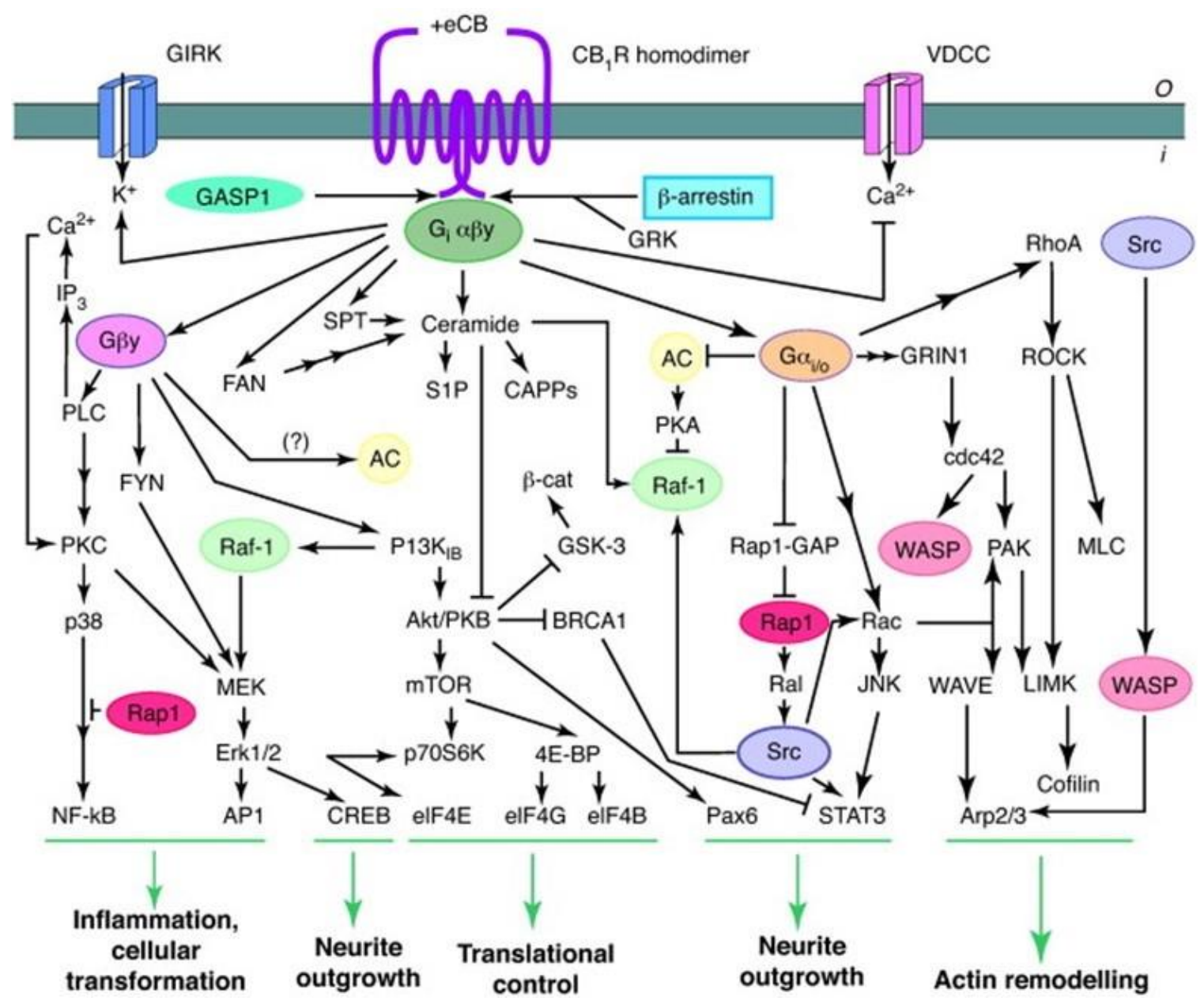

Figure 1-1. CB1R signaling pathways.

Agonist-induced signaling at the $\mathrm{CB}_{1} \mathrm{R}$. Reprinted from Trends in Pharmacological Sciences, 32(9), Keimpema, E., K. Mackie, and T. Harkany, Molecular model of cannabis sensitivity in developing neuronal circuits, 551-561, Copyright (2011), with permission from Elsevier. 
neurotransmitters and hormones. Cyclic AMP has three main downstream effectors: cAMP-dependent protein kinase (PKA), a guanine nucleotide exchange factor (GEF) known as EPAC, and various cyclic nucleotide gated ion channels [35]. PKA is the prominent and best studied effector with involvement in regulating metabolic enzymes and transcription factors, regulating other pathways such as the PIP2 pathway through control of phospholipase $\mathrm{C}$, and contributing to ion channel permeability [35]. This allows PKA to influence widespread cellular and physiological functions, all of which are subject to control of CB1R by agonistic activation through cannabinoids reducing cAMP levels.

Ion channel regulation is one consequence of $\mathrm{CB} 1 \mathrm{R}$ activation. One ion known to be regulated by cannabinoid signaling is potassium, which is manipulated through the activation of G-protein coupled inwardly-rectifying potassium channels (GIRKs) within this pathway and induction can occur through activation by multiple CB1R agonists [36, 37]. Specifically, GIRK1 and GIRK4 channels have been reported to have active responses in the presence of CB1R signaling in amphibian oocytes [38] as well as neuronal cells of murine models $[39,40]$. When these channels open, potassium is removed from the cell, decreasing its intracellular concentration [41]. The second ion known to have downstream effects due to CB1R signaling is calcium. While some calcium regulation is cell type specific with unknown mechanisms of response [42], several CB1R regulatory mechanisms for calcium have been defined. Insulinoma cells can regulate calcium in a cannabinoid-dependent manner through the involvement of $\mathrm{Gq}$ proteins and phospholipase $\mathrm{C}$ activity induced by cannabinoid agonist arachidonoylchloro-ethanolamide, JWH133, and anandamide [43]. Voltage-gated ion channels can be influenced by CB1R stimulation. L-type calcium channels in cerebral vessels [44], retinal bipolar cells [45], and neonatal rat solitary tract cells [46] were inhibited in the presence of cannabinoids. In contrast, CB1R induced activation of L-type calcium channels has been demonstrated in neuroblastoma cells [47]. Numerous studies provide evidence of CB1R's ability to inhibit P/Q-type calcium channels [37, 48-51] as well as N-type calcium channels $[39,50,52-56]$ due to CB1R signaling, which could be important in cannabinoid presynaptic inhibition and retrograde signaling $[57,58]$.

The MAPK pathway, an important signaling cascade involved in many cellular functions, can be activated through agonistic activity of GPCRs. These pathways have a long signaling ladder of kinase activity which lead to activation of many pathways. One of these pathways leads to the activation of extracellular signal-related kinases 1 and 2 (ERK1/2). ERK1/2 participates in the Ras-Ref-MEK-ERK signaling pathway which involves a large number of physiological processes in the cell including cell movement, metabolism, cell cycle, cell survival, cell differentiation, cell proliferation, and transcription [59]. CB1R stimulation can activate ERK1/2 in multiple cell types and could be accomplished through a variety of mechanisms including activation of $\mathrm{Gi} / \mathrm{o}$ proteins [58], inhibition of PKA through AC [60], phosphatidylinositol 3-kinase (PI3K) through the PIP2 pathway [61], transactivation of other receptors such as vascular endothelial growth factor (VEGF) or tyrosine kinase FYN $[62,63]$. Other MAPK pathways linked to CB1R stimulation are the JKN and p38 MAPK pathways [64, 65]. Both pathways are involved in cell apoptosis and inflammation, with JNK having 
addition roles in metabolism and influence on mitochondrial proteins and p38 MAPK being involved in the cell cycle and cell differentiation [66-70]. JKN and p38 MAPK pathways both primarily respond to stressful or harmful phenomenon such as cytokines, ultraviolet light, heat shock, osmotic shock, radiation, senescence, and tumorigenesis [68, 71]. Activating MAPK pathways can be dependent on cell or tissue type. CB1R activity can invoke ERK1/2, p38 MAPK, and JNK pathway response in endothelial cells [64], where cannabinoids were only able to activate the p38 MAPK pathway in hippocampal slices [63] and JNK in cultured cortical neuron cells [72].

With GPCR induction, there are also unique signaling transduction events tied to the proteins responsible for their internalization/desensitization, $\beta$-arrestins. In addition to this important function, $\beta$-arrestins have other roles in signaling and possess their own signaling pathways. One prominent signaling role is their function as scaffolding proteins $[73,74]$. When acting as a scaffold, $\beta$-arrestin places specific proteins in close proximity and occasionally in certain locations within the cell. This increases efficiency of pathway transduction, decreases unwanted, alternate pathway reactions, and brings effector proteins to needed areas of a cell. Working as a scaffold also allows for influence on basal activity levels, and evidence exists that arrestin scaffolds are key regulators of cell growth, migration, and survival pathway [75]. $\beta$-arrestin also can bind to transcription factors such as NFKB and p53 [76, 77] and cofactors such as p300 [78]. While bound to $\beta$-arrestin, CB1R can activate alternative signaling pathways in a G-protein independent manner through $\beta$-arrestin $[79,80]$. This has been supported with evidence that increased interaction of $\beta$-arrestin to CB1R surface proteins results in an increase in $\beta$-arrestin mediated signaling [81]. While bound to receptors, it is proposed that selective signaling could be achieved with downstream $\beta$-arrestin pathways by isolating G-protein bound signaling cascades over $\beta$-arrestin [82]. This is evident with the $\mu$-opioid receptor, a GPCR that binds to Gi/o proteins and recruits arrestins, much like CBRs. These results point to strong therapeutic potential having fewer adverse side effects when biased towards G-protein coupled signaling [83-85]. It is possible that CBRs could hold this same potential with drug response while producing less negative effects. Agonist designed to be biased towards G-protein bound and away from $\beta$-arrestin bound signaling have begun clinical trials $[86,87]$.

\section{Cannabinoid Receptor 2}

The majority of cannabinoid ligands that induce activity with CB1R also stimulate CB2R, albeit with different efficacy with most ligands [11]. The signal transduction pathways of CB2R are not characterized as well as CB1R. However, it is known to share some of the same signaling mechanisms such as coupling to the $\mathrm{G}_{\mathrm{i} / \mathrm{o}}$ subunit to inhibit AC, MAPK activation, and intracellular calcium regulation through phospholipase C activity [88-90]. CB2R does not seem capable of regulating potassium through GIRK channels or calcium through voltage gated mechanisms like CB1R [91]. As with the $\mathrm{CB} 1$ receptor, $\mathrm{CB} 2 \mathrm{R}$ also undergoes agonist-mediated phosphorylation, followed by a decrease in signaling and surface receptor levels $[92,93]$. $\beta$-arrestin- 2 , the same arrestin that interacts with $\mathrm{CB} 1 \mathrm{R}$, is also believed to be the cause of the 
internalization and desensitization of this receptor. A fascinating characteristic of CB2R is its high inducibility, with expression able to increase up to 100 fold in the presence of inflammation or tissue injury [94].

\section{Non-Cannabinoid Receptor Activity}

Several GPCRs other than CB1R and CB2R are capable of cannabinoid ligation and have been suggested to be cannabinoid receptors themselves. To date, only two have been verified to have cannabinoid activity: GPR18 and GPR55 [95, 96]. GPR55, which is stimulated by cannabinoids such as THC, anandamide, 2-AG, JWH015, and CBD in HEK293 cells [97], is expressed throughout the body and co-localizes with CBRs in various areas $[98,99]$. It's G-protein association is dynamic, coupling to $\mathrm{G}_{13}, \mathrm{G}_{\mathrm{q} / 11}, \mathrm{G}_{12}$, or $\mathrm{G}_{12 / 13}$ depending on cell or tissue type [97, 100-102]. When coupled to $\mathrm{G}_{\mathrm{q} / 11}$ subunits, GPR55 provokes calcium signal generation, increasing intracellular calcium levels [97]. Activation of GPR55 is linked to calcium signaling in endothelial cells, but this activity was dependent on the stimulation of CB1R [103]. With GPR55 and CB1R having overlapping ligand specificity it is possible that some calcium signals created by cannabinoid activity could be mediated by GPR55. GPR18 responds to THC, abn-CBD and the anandamide metabolite NAGly $[95,104]$ and associates with $\mathrm{G}_{\mathrm{i} / \mathrm{o}}$ and $\mathrm{G}_{\mathrm{q} / 11}$ subunits $[105,106]$. Expression of GPR18 is the highest in spleen and bone marrow with lower expression in lungs, brain, testis, and ovary [105, 107]. GPR18 inhibits cAMP production due to its coupling to $\mathrm{G}_{\mathrm{i} / \mathrm{o}}$ [105], and has been implicated in cell migration [104] and apoptosis [108]. The lack of strong, selective agonist for these receptors hampers a deeper understanding of these receptors and development of biased agonist may be needed for further investigations.

Cannabinoids also have targets other than GPCR receptors. They bind and activate transient receptor potential (TRP) channels, which are cation channels typically activated by heat or capsaicin and cold or methanol depending on the TRP channel [109, 110]. Ligand-induced activation of these channels leads to feelings of temperature sensation followed by a period of increased antinociception. Endocannabinoids anandamide and 2-AG are capable of activating TRPV1 channels while THC is capable of activating TRPV2, a heat activated channel, and TRPA1, a cold activated channel $[109,111,112]$. These TRP channels co-express with CB1R in multiple areas of the CNS, making it plausible that endocannabinoids might be naturally producing physiological effects through these channels $[111,113,114]$. A second non-cannabinoid receptor type activated by cannabinoids are nuclear receptors known as PPARs (peroxisome proliferator activated receptors). THC has affinity for PPAR $\gamma$, which has insulin-like effects on metabolism, but also has evidence of affecting expression of the CB1 receptor, potentially playing a role in the endocannabinoid system [115]. PPAR $\alpha$, inducible by anandamide and 2-AG, reduces appetite and brings forth analgesic and neuroprotective responses [116]. Further investigation is warranted to understand if these non-cannabinoid receptors play a more extensive role in the endocannabinoid system. 


\section{Homo- and Heterodimerization of CB1R}

Physical interaction and cooperative function between surface proteins was speculated back in the 1970s [117] and since then an abundant amount of data has been acquired demonstrating GPCRs functioning as both homo and heterodimers [118-122]. When forming dimers, there are often changes in receptor function leading to alterative signaling pathways [122]. CB1R is capable of homodimerization [123] as well as heterodimerization with D2 dopamine receptors [124], A2A adenosine receptor [125], $\beta 2$ adrenergic receptor [123], and opiate receptors [126]. Heterodimerization with D2 dopamine receptors provides evidence pointing towards the function of the heterodimer relying on CB1R stimulation [124]. When bound to D2 receptors, CB1R leads to an increase in intracellular CAMP concentration rather than a decrease [28]. This is believed to be caused by a change in CB1R's G protein association, from its normal Gi/o subunit to Gs [127]. This gives rise to the possibility of having stable, active CB1R surface proteins coupled to Gs. When forming a dimer with A2A adenosine receptor CB1R cannot activate is Gi/o protein independently, but needs activation of A2A to perform its function [128]. The $\mu$-opioid/CB1R heterodimer is capable of effective Gi/o signaling when either receptor is stimulated alone, but Gi/o signaling is inhibited when they are coactivated [126]. Dimerization of CB1R and other GPCRs may be a useful model to explain the altered functional properties and signal transductions that these receptors are capable of performing under varying circumstances, such as in differing tissues or under pathological conditions.

\section{Distribution of Cannabinoid Receptors}

\section{Neuronal Presence of CB1R}

In the CNS, CB1R is located mainly at presynaptic sites and along axons, allowing them to control neurotransmitter releases at synapses and influence neuronal function [129-131]. However, there is evidence with self-inhibition studies using endocannabinoids on neocortical neurons that CB1R could be present at postsynaptic sites $[132,133]$. Using molecular techniques and conditional knockout mice, studies demonstrate that cannabinoid receptors have both excitatory and inhibitory neurotransmission effects due to their location on both GABAergic and glutamatergic neurons $[134,135]$. However, alterations in mental state and certain physiological responses is a result of agonistic cannabinoid binding to CB1R surface proteins of glutamatergic principal neurons in the CNS [134]. CB1 receptors have been reported to have varying concentration levels depending on cell type. Some of the highest levels of CB1R were detected in the cortex and hippocampus, particularly on cholecystokinin interneurons [136-138], but they are widely distributed at lower levels in glutamatergic cells [135]. Within the striatum, an abundance of the CB1 receptor can be found in medium spiny neurons [139-141]. While its presence in neurons is of critical importance to $\mathrm{CB} 1 \mathrm{R}$ function, it is also present and has functional relevance in glial cells $[142,143]$, 
which is not all that surprising with the discovery of microglial cells and astrocytes being capable of producing their own endocannabinoids, specially 2-AG and anandamide [144].

\section{CB1R Distribution}

The $\mathrm{CB} 1$ receptor was first discovered in the brain and was then categorized as the most abundant GPCR there with the highest expression levels detected in the cortex, striatum, hippocampus, and cerebellum and lower levels seen in the thalamus, hypothalamus, brainstem, and spinal cord [145]. This distribution of CB1R in the CNS corresponds with some of the behavioral effects caused by receptor activation. Its influence on motor function and coordination are likely mediated by receptor activity in the cortex, striatum, and cerebellum [146, 147]. The striatum and areas of the cortex also play a role in the cataleptic traits of cannabinoid activity [134, 148]. Hypothermic response has been linked to hypothalamic mediation based on injections of cannabinoids directly into the tissue $[149,150]$, while cannabinoid antinociception response may depend primarily on spinal mechanisms [151]. Reviewing the effects of cannabis use and CB1 receptor levels together reveals some discrepancies. Cannabinoid activity effects an individual's homeostatic state [146], including temperature regulation, but CB1 receptor levels are low in the hypothalamus, a region of the brain believed to modulate many of these processes [152]. The low level of receptors is believed to be compensated by a higher efficacy of CB1 in the hypothalamus. This was concluded by comparing CB1R activity to relative levels of the receptor present [153]. The efficiency of the CB1 receptor varies in other regions of the brain, even within different areas of the cortex with similar expression levels [154]. When comparing different areas of the brain, the hypothalamus has some of the highest CB1R efficiency, which would allow fewer receptors to achieve behavioral or physiological effects [155].

CB1R is also present in the peripheral nervous system (PNS) [145]. Within the PNS CB1R has been located in sympathetic neurons, trigeminal ganglion, dorsal root ganglion, and primary sensory cells and has the ability to regulate pain [156-159]. One peripheral region $\mathrm{CB} 1 \mathrm{R}$ is present in is the enteric nervous system and intestine inner lining of the GI tract where it contributes to control of secretion of acids and neurotransmitters, tract mobility, and epithelium permeability [160]. The receptors are also expressed in the liver, but at very low levels under normal conditions [161] with the ability to increase expression under pathological conditions and are involved in insulin resistance, fibrosis, and lipogenesis [162]. Lower levels of CB1R expression are found in the cardiovascular system as well where they help modulate heart rate and blood pressure [163]. Other tissues and areas expressing detectable levels of CB1R are adipose, bone, muscle, skin, eye, and the reproductive system [162].

\section{CB1R Subpopulations}

While most CB1R is primarily localized to the cell surface and participates in $\mathrm{G}$ protein-dependent signaling, intracellular localization of $\mathrm{CB} 1 \mathrm{R}$ has also been reported 
with characteristics of functional subpopulations [23]. One subpopulation is located in various endosomes and lysosomes and believed to be independent from normal receptor activity, meaning separate from the population of constitutive and agonist bound internalized membrane receptor population $[22,164]$. This distinct endo/lysosome CB1R group, upon intracellular agonist activation, increases the release of calcium from both the receptor's vesicle and the endoplasmic reticulum [165]. A second group of CB1 receptors independent from plasma membrane receptor activity are located in the mitochondria. THC has been reported to have effects on mitochondria associated enzymatic activity through the presence of mitochondrial CB1R and its involvement in cellular respiration $[166,167]$. Many other roles of mitochondrial CB1 have been investigated including cannabinoid-induced hunger, memory impairment, and cerebral ischemia/reperfusion injury, suggesting a larger role in its contribution to mitochondrial activity [168-170]. The presence of mitochondrial CB1R is believed to be important for proper function and overall health of mitochondria, potentially effecting mitochondrial related pathological conditions [171-173].

\section{CB2R Distribution}

CB2R was first discovered in immune tissue and later studies confirmed that the predominant location of CB2R is in immune cells $[8,174]$. Here CB2R activation effects adaptive immune response by negatively regulating cell migration and producing inflammatory factors such as cytokines and chemokines [175, 176]. The CB2 receptor is present at a much lower level in the CNS and PNS [177] and is primarily located in microglia and vascular elements of the nervous system rather than neurons $[178,179]$. A stronger presence of CB2R in neurons under pathological conditions have been noted $[180,181]$. Even with its low expression within the nervous system, CB2R contributes to neurological function, such as nociception, addiction, and neuroinflammation [182, 183]. Lines of evidence exist that $\mathrm{CB} 2 \mathrm{R}$ can regulate neuron excitability through of control of specific chloride channels in certain brain tissue [184]. It also has moderate expression in some peripheral tissues, many overlapping with CB1R expression - cardiovascular, gastrointestinal, liver, adipose, bone, and reproductive [174].

\section{Prevalence, Use, and Effects of Cannabis}

\section{Use, Legalization, and Social Outlook}

Cannabis is one of the most widely used drugs in the United States and its use continues to increase, which is especially true in states where recreational use of cannabis has been legalized [185, 186]. Currently (as of July, 2020) 34 States have legalized cannabis for medical use and 11 of those States have also legalized recreational use. With increasing support from the general public and legal precedent being set by individual states throughout the U.S., it can be assumed that the legalization of cannabis will continue to spread throughout the country, further increasing the number of users and use 
frequency. New legislation isn't the only change in cannabis in recent years. The concentration of THC in marijuana has tripled in the U.S. over the last two decades from $4 \%$ to $12 \%$, potentially intensifying adverse effects associated with use [187]. The general perception of cannabis use, both medical and recreational, is becoming more acceptable in the U.S. $[188,189]$ and there is even a decrease in belief that cannabis use puts the individual at risk of harm $[190,191]$.

\section{Physiological Effects and Pathological Roles of Cannabis and Its Receptors}

Physiological effects of using cannabis are seen throughout different systems of the body. The effects on the CNS include changes in analgesia, neuronal excitability, and neuroprotection [192-194]. CB1Rs modulate neurotransmission by inhibiting the neurotransmitters GABA and glutamate in their respective neurons. Alleviating pain is one of the earliest therapeutic uses of cannabis and many studies focus on its effectiveness with different types of pain $[195,196]$. While CB1R plays the most prominent role in regulating nociception, $\mathrm{CB} 2 \mathrm{R}$ may also play a role in cannabinoid regulation of pain [197]. The neuroprotective effect of CB1R stimulation, which allows it to potentially play a role in neurological disorders such as epilepsy and excitotoxicity, has been linked to glutamatergic neurons $[198,199]$. Other than its main function of inhibiting neurotransmission, there is evidence that CB1Rs neuroprotective ability extends to other biological functions such as inhibiting nitric oxide production and increasing expression of the neuronal survival and growth protein BDNF [200, 201]. Alterations in the endocannabinoid system, including changes in CB1R expression, are present in neurodegenerative diseases, such as Huntington's, Parkinson's, and Alzheimer's [193]. Animal models of Alzheimer's study how induction of CB1R can potentially benefit memory deficits and other cognitive dysfunctions that come with the disease [202, 203]; with the underlying cause likely due to CB1R preventing amyloid $\beta$ neurotoxicity [204-207]. Loss of CB1R expression can be an early sign of Huntington's [208] and mouse models suggest therapeutic potential with administration of THC partially alleviating some of the disease's symptoms [209].

Physiological effects in peripheral regions include changes in heart rate, blood pressure, and appetite along with decreases in nausea, intraocular pressure, body temperature, and inflammation [162]. With their presence in the cardiovascular system both CBRs possess the ability to modulate basic cardiac function such as heart rate and blood pressure [163]. Pathological upregulation of CB1R occurs in the cardiovascular system, but with harmful effects. Following CB1R upregulation, oxidative stress, inflammation, and fibrosis have been observed throughout various cell types of the cardiovascular system which can further progress cardiac disease, dysfunction, and damage [163]. On the other hand, CB2R seems to have protective attributes with cardiovascular disease such as inhibiting fibrotic proliferation, decreasing inflammation, and exerting positive effects in cardiomyocytes [210]. When using cannabis, a commonly known side effect is an increase in appetite commonly referred to as the "munchies". This is believed to be driven by CB1R activity in the hypothalamus with endocannabinoid 
levels increasing during fasting and returning to normal after eating and appetite levels can be diminished by CB1R antagonists [211, 212].

The broad spectrum of effects associated with cannabis use is not surprising when considering the widespread distribution of its receptors. Cannabis's endogenous receptor CB1R has differing roles across many pathological conditions with both positive and negative consequences related to CB1R expression and function [161]. Pharmacologically activating the receptor with agonists can even have phasic effects along with unwanted side effects due to CB1Rs widespread distribution, illustrating the need for better understanding of ECS signaling and regulation [213].

\section{Behavioral Effects and Psychology of Cannabis Use}

As mentioned earlier, THC is believed to be the psychoactive component primarily responsible for many of the effects seen with cannabis use, including the negative behavioral and cognitive consequences. Behavioral effects seen with people under the influence are alteration of mood, an increase in sedation, low levels of attention and alertness, and increases in anxiety while cognitive include faulty judgment and perception, memory impairment, difficulty learning, and motor skill dysfunction [214].

Chronic use of cannabis can negatively affect brain development, decrease academic performance and future achievements, and puts the user at high risk of developing mental illness [194, 215, 216]. Accumulating genetic evidence suggests a link between polymorphisms of cannabinoid receptors and other ECS genes and an increased risk of developing schizophrenia [217-220].

\section{Abuse and Dependence}

As with other drugs of abuse, cannabis users are at risk of developing dependency to the drug, known as cannabis use disorder (CUD) [221]. Drug abuse is a major health, economic, and social concern that effects the entire population. According to the most recent US survey of National Drug Use and Health there were 3.2 million new users of illicit drugs over the age of 12 in 2017 compared to 2016; of which the overwhelming majority were new cannabis users [185]. Currently, approximately 1 in 10 users of cannabis become addicted [222] and that rate nearly doubles for those who start using as adolescents [223]. As the number of users, frequency, and drug potency increase, a potential consequence could be an increase in both the number and severity of adverse health effects seen among cannabis users, including a higher prevalence of CUD [224].

Numerous factors contribute to drug use and the development of dependence. Non-drug related factors are social and environmental such as stress, depression, impulsivity, and peer pressure [225, 226]. Drug related factors are sensitivity and tolerance [227]. One aspect of drug use that has been linked to addiction is the balance of positive and negative effects that a new user undergoes during their first exposure. 
Individuals who experience a high degree of positive effects, such as the feeling of euphoria or relaxation, are more likely to develop addiction than those that experience limited positive effects or negative effects. Studies reporting subjective responses from users have been done with a variety of drugs, including opioids [228], caffeine [229], cocaine [230], and cannabis [231, 232]. Respondents from these studies who report feeling the strongest positive effects have a shorter period before second exposure as well as higher lifetime use, with a risk of progression to drug dependence. For cannabis, users with the greatest positive responses were 28 times more likely to develop dependence as those who did not experience positive effects during their first exposure [232]. These studies provide evidence that a positive subjective experience with initial use of a drug could be predictive of future use and addiction.

There is also evidence suggesting that the level of sensitivity an individual has to a drug during their first exposure can affect subsequent use. Those who are naturally insensitive have a heightened risk for abusing the drug later in life. Rodent models have demonstrated this with morphine [233], alcohol [234, 235], and nitrous oxide [236], and retrospective human studies corroborate this by establishing parallels between initial insensitivity upon first exposure and increased use and dependence for opioids [228], alcohol [237, 238], and cocaine [230]. Genetic factors are believed to be an important determinant in observed differences in drug response and abuse [239-241]. CUD is believed, in part, to be heritable and influenced by genetics [242, 243]. Genes that have been linked to cannabis use and CUD are limited [244-247], leaving undiscovered what genetic mechanisms and physiological processes are contributing to initial sensitivity and development of abuse.

While attempts are being made to better understand the biochemistry of the nervous system in response to cannabis use and develop targeted pharmacotherapies to treat dependence, no clearly effective treatments have been established [248]. This is in part due to a lack of a deeper understanding of the biochemical response of cannabis use, particularly at the level of the individual. Developing preventative strategies for drug addiction could go a long way in helping vulnerable, high-risk individuals.

\section{Variation in Response}

The more we learn about drug use, the less doubt there is that variation is seen amongst individuals in their responses to drugs of abuse, including cannabis [231, 249]. Human studies using intravenous THC showed dramatic differences among individuals to the primary psychoactive component of the drug [250, 251]. These differences illustrate that underlying genetic mechanisms could be driving this fluctuating response.

Administration of cannabis, THC, and other cannabinoids in murine studies have also been quantified among genetically distinct inbred strains of mice, including ddY, ICR, Swiss Webster, C57BL/6J (B6), DBA/2J (D2), and C57BL/6JArc strains [252-255]. These animal model studies further support the evidence seen in humans that response to cannabis and/or its components can vary and genetic differences among individuals are, in part, governing the response. 


\section{CHAPTER 2. SEX AND STRAIN VARIATION IN INITIAL SENSITIVITY AND RAPID TOLERANCE TO $\triangle$ 9-TETRAHYDROCANNABINOL ${ }^{1}$}

\section{Introduction}

Initial response to cannabis, THC, and other CB1R agonists has been measured across a range of doses among several inbred and outbred mouse strains [252-261]. However, few of these studies have directly compared strain differences in response and most do not include females, thus preventing analysis of sex differences. Despite marked variation among individuals in the acute response to cannabis in humans [249], little is known about the genetic factors mediating differential response or how initial sensitivity and tolerance may contribute to risk of CUD. Few studies systematically address possible genetic or sex differences in both initial response and tolerance to cannabinoids in human or animal models.

In this study we begin to address these gaps in knowledge by profiling the response to the main psychoactive substance in cannabis, THC, in males and females from highly genetically divergent C57BL/6J (B6) and DBA/2J (D2) inbred strains of mice. These strains differ at millions of loci [262] and are the progenitor strains for a large and well characterized panel of recombinant inbred strains, the BXD population [263]. Many phenotypes exhibit heritable (controlled by genetic factors) differences among the B6 and D2 parental strains and their recombinant inbred BXD progeny, including response to drugs of abuse - ethanol, nicotine, methamphetamine, cocaine, and opioids [264-268]. The gene variants underlying these and other heritable differences between B6 and D2 can be identified using forward genetic mapping approaches in the BXD panel [269].

To begin to address potential genetic variation in specific molecular signaling pathways that mediate response to THC, we measured behavioral and physiological responses directly related to THC activation of CB1R in male and female B6 and D2 mice. These responses include diminished locomotor activity, hypothermia, and longer latencies to respond to a thermal stimulus [270-274]. Specifically, we quantified initial sensitivity as the acute response upon first exposure to THC and rapid tolerance as the response upon the second exposure to THC.

Rapid tolerance was included because repeated daily administration of THC in humans and animals produces rapidly diminished physiological responses (rapid tolerance) within just a few days. This response may be linked to the rapid adaptation or desensitization of the main neuronal receptor of the endocannabinoid system, CB1R

\footnotetext{
${ }^{1}$ Reprinted from final submission with permission. Parks C, Jones BC, Moore BM, and Mulligan MK. Sex and strain variation in initial sensitivity and rapid tolerance to $\Delta 9-$ tetrahydrocannabinol. Cannabis and Cannabinoid Research, 2020 5(3). http://doi.org/10.1089/can.2019.0047 [275].
} 
[276]. We also compared overall rates of tolerance/desensitization following five consecutive daily exposures to THC.

In this study we provide the first report of sex differences in the hypothermic response to THC and genetic (strain) differences in initial sensitivity and rapid tolerance to the locomotor and/or analgesic effects of THC. Identification of genetic differences influencing sensitivity and tolerance to THC is important for understanding negative aspects associated with acute (e.g. motor depression and intoxicating effects) and chronic use (e.g. cannabinoid dependence and withdrawal), and for developing the therapeutic potential (e.g. analgesic effects) of the endocannabinoid system.

\section{Materials and Methods}

\section{Animals}

B6 and D2 mice (at least 20 per strain) were purchased from the Jackson Laboratory or bred in house as described below. All mice were aged to at least 60 days prior to testing. At least one week prior to testing, animals were separated into individual housing and handled daily. To reduce stress and anxiety responses throughout the study, handling (with the exception of i.p. injections) consisted of lifting mice in either cupped hands (avoiding any lifting by the tail) or a plastic lid from a pipette tip box [276-278]. Food and water were provided ad libitum and mice were maintained on a $12 \mathrm{~h}: 12 \mathrm{~h}$ light:dark cycle with lights on at $0600 \mathrm{~h}$. All testing was performed during the light cycle from $0700 \mathrm{~h}$ to $1600 \mathrm{~h}$. All animal activities were approved by the University of Tennessee Health Science Center Institutional Animal Care and Use Committee.

\section{THC and Vehicle Formulation}

THC was formulated in an ethanol:cremophor:saline (5:5:90) Vehicle (VEH) followed by filter sterilization. The resulting formulation was stored in the dark and under $\left(4^{\circ} \mathrm{C}\right)$ refrigeration in a septum sealed vial. A new formulation was generated for each cohort of mice and used within 1 week of formulation. The VEH was prepared in the same manner. THC and VEH were administered by intraperitoneal (i.p.) injection at a dose of $10 \mathrm{mg} / \mathrm{kg}$ such that a $30 \mathrm{~g}$ mouse received a $100 \mu \mathrm{L}$ injection. The $10 \mathrm{mg} / \mathrm{kg}$ dose was selected based on previous preclinical studies that demonstrate significant responses (hypolocomotion, hypothermia, and/or antinociception) in a number of strains at this dose [252-261].

\section{THC Formulation Stability}

Five $\mu \mathrm{L}$ of the formulation was removed immediately after preparation and 8 days post preparation and each diluted with 100 microliters of methanol. The samples were 
analyzed immediately following sample preparation using high-resolution mass spectroscopy (HRMS) on a Waters Xevo G2+S QTOF (Milford, MA) system in negative mode.

A reversed-phase BEH C18 analytical column $(2.1 \mathrm{~mm} \times 50 \mathrm{~mm}, 1.7 \mathrm{~mm}$ particle size, Waters, Milford, MA) was used for the LC separation. The mobile phase was $95 \%$ water with 5\% acetonitrile (solution A) and acetonitrile (solution B). A 5 min gradient elution of $20 \%$ solution $\mathrm{B} ; 1.5 \mathrm{~min}-3.0 \mathrm{~min}, 20 \%$ to $100 \%$ solution $\mathrm{B} ; 3.0 \mathrm{~min}-4 \mathrm{~min}$, $100 \%$ solution B; 4 min -4.5 min $100 \%$ to $20 \%$ solution B back to initial condition and finish at $5 \mathrm{~min}$ and a flow rate of $0.3 \mathrm{~mL} / \mathrm{min}$.

A PDA detector (Waters, Milford, MA) was used to measure the UV chromatogram before mass spectrometer analysis. The THC eluted at 3.75 minutes with a molecular mass of 313.2017 [M-H] (calculated for m/z C21H29O2 [M-H] 313.2173). The THC peaks were integrated and the formulation stored for 8 days contained $95.2 \%$ of the initial THC used in the formulation.

\section{Phenotyping Pipeline A}

An initial pilot study was performed first in B6 and D2 male mice (20 per strain) purchased from the Jackson Laboratory. A separate cohort of female mice (18 per strain) also purchased from the Jackson Laboratory was tested $\sim 1$ year later. Mice were randomly assigned to the THC or VEH groups. The number of mice tested in this pipeline were as follows: 4 VEH B6 females, 4 VEH D2 females, 4 VEH B6 males, 4 VEH D2 males, 14 THC B6 females, 14 THC D2 females, 16 THC B6 males, and 16 THC D2 males.

Mice were tested over seven consecutive days (Figure 2-1). No injections were given on day -1 (acclimation). On day 0 (baseline) all subjects received a VEH injection $(100 \mu \mathrm{L}$ per $30 \mathrm{~g}$, i.p.) and on days 1 through 5 (treatment) all subjects received a VEH or THC injection (i.p.). On each day, body temperature and motor activity were measured at multiple time points (Figure 2-1). Because repeated testing within session can influence behavior, tail withdrawal latency in response to a thermal stimulus was measured at a single time point (60 minutes). Time points and THC dose $(10 \mathrm{mg} / \mathrm{kg}$ ) were selected based on literature survey. Previous studies demonstrated that $10 \mathrm{mg} / \mathrm{kg}$ (i.p.) THC is the lowest dose that reliably produced significant effects in locomotion, hypothermia and/or antinociception relative to controls across a range of mouse strains (predominately male)[252-261].

Temperature was measured using a ThermoWorks digital thermometer with rectal probe adaptor at time 0,30 , and $60 \mathrm{~min}$ post-injection. Analgesia was measured $60 \mathrm{~min}$ post-injection as the tail withdrawal latency to a thermal stimulus using a tail flick assay. In this assay, mice were gently restrained in a $50 \mathrm{ml}$ conical tube or by hand when mouse would not remain calm in tube, and the tail was submerged approximately $2 \mathrm{~cm}$ in a $52^{\circ} \mathrm{C}$ water bath. The latency to remove the tail 

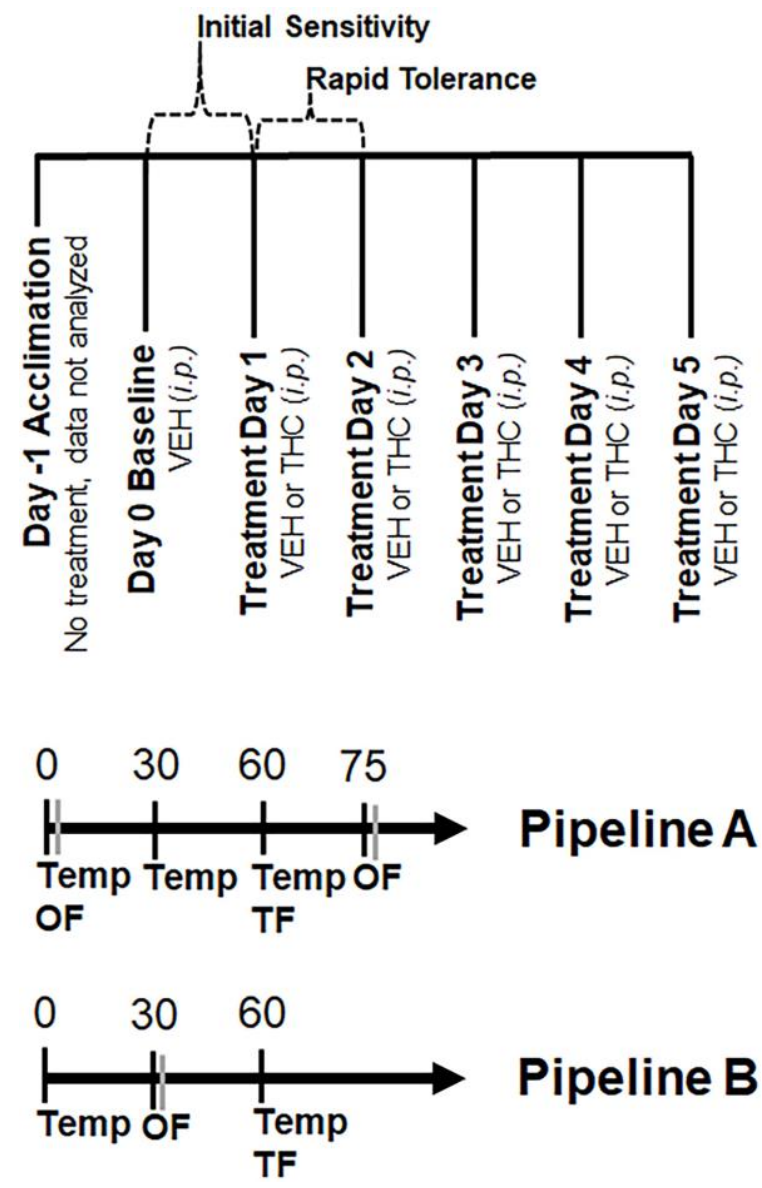

Figure 2-1. Overview of phenotype testing pipelines.

An overview of treatments and test days is in the top panel. Testing occurred over 7 days. On the first day, mice were acclimated to the testing paradigm for testing pipelines $\mathrm{A}$ and B. Animals were not treated and data collected on this day were not analyzed. Baseline data were collected on day 0 and all mice received a VEH injection (100 $1 \mathrm{~L}$ per $30 \mathrm{~g}$ body weight, i.p.). On days 1 through 5, mice received either VEH or THC (10 mg/kg, i.p.) treatment depending on their random assignment to either condition. At the indicated time post-injection of VEH or THC, and on each day, mice were tested for the effect of treatment on hypothermia (body temperature or Temp), locomotion (spontaneous motor activity over $10 \mathrm{~min}$ in the open field or $\mathrm{OF}$ ), and analgesia/antinociception (tail withdrawal latency to a thermal stimulus or TF). VEH, vehicle; THC, D9tetrahydrocannabinol. 
in response to the thermal stimulus was recorded to the nearest 1/10 second by stopwatch. Average tail flick latency was recorded based on two independent observers.

Motor activity was measured as the time spent mobile (spontaneous motor activity) in a $40 \mathrm{~cm}$ x $40 \mathrm{~cm}$ open field at 0 and 75 min post-injection. Mice were gently transported by hand or in a clear plastic box to the center of the open field and activity was video-recorded for 10 min. Immobility was detected using AnyMaze (Stoelting) software and was operationally defined as no movement for at least $3 \mathrm{sec}$. Mice were habituated to the test room for one hour prior to testing.

\section{Phenotyping Pipeline B}

Male and female mice from a breeding colony at UTHSC (less than six generations from the Jackson Laboratory stock) were used. Mice were randomly assigned to THC or VEH groups. Males and females of both strains were tested together in several small batches. Strain and sex were counterbalanced across batches and within daily sessions. Up to eight mice were included in each session and up to four sessions were run per testing batch. Mice were returned to their home cage between tests. Mice were habituated to the test room for one hour prior to testing.

The testing schedule was similar to pipeline A with the exception that the schedule was streamlined to increase throughput by measuring phenotypes at a single time point (Figure 2-1) over a shortened time period $(60 \mathrm{~min})$ relative to pipeline A (85 min). Mobility in the open field was measured at 30 min post-injection and core body temperature and analgesia were measured at $60 \mathrm{~min}$ post-injection. The number of mice tested in this pipeline were as follows: $6 \mathrm{VEH}$ B6 females, $6 \mathrm{VEH}$ D2 females, $12 \mathrm{VEH}$ B6 males, 14 VEH D2 males, 16 THC B6 females, 13 THC D2 females, 17 THC B6 males, and 17 THC D2 males.

\section{Statistical Analysis}

Data from all cohorts and both phenotyping pipelines were combined for statistical analysis. Statistical outliers (greater than 2 standard deviations from the mean for each sex and strain by day and trait) were excluded from further analysis as were individuals with technical artifacts (e.g. bad injection, AnyMaze software failure, temperature probe failure, or mouse with obvious health issues). Data from days 0 through 5 were included in the analysis. Raw trait data (mobility and tail flick latency) for each individual was transformed relative to baseline (day 0) by subtracting trait values for each day by the trait values for day 0 . Raw temperature trait data for each individual was transformed relative to baseline (time 0 ) by subtracting trait values for each day (time 30 or 60) by trait values for time 0 .

For each individual, response to treatment (THC or VEH) was calculated as the area under the curve (AUC) using the plyr and MESS packages in R. The spline function 
[type $=\mathrm{c}($ "spline")] and the absolute area (negative and positive areas combined; absolutearea=TRUE) were used with MESS package defaults for the auc function in order to calculate AUC.

Omnibus ANOVAs were then performed on the AUC data using base functions in $\mathrm{R}$ in order to determine trait response based on a number of independent factors. Time mobile in the open field (a measure of the motor depressant effect of treatment), was evaluated using a multi-factor ANOVA: AUCmobile Treatment*Strain*Time*Sex. Body temperature (a measure of the hypothermic effect of treatment), was evaluated using a multi-factor ANOVA: AUCtemperature Treatment* Time* Sex*Strain. Tail flick latency to a thermal stimulus (a measure of the antinociceptive effect of treatment), was evaluated using a multi-factor ANOVA: AUCtemperature Treatment*Strain*Sex. Factor order was arranged to maximize impact (F-value) of each factor on trait variation.

Post-hoc analysis [multifactor ANOVA and Tukey's honest significant difference (HSD)] was then performed to specifically evaluate the effect of sex and strain on initial sensitivity and rapid desensitization to THC as described below.

Initial sensitivity was calculated for the THC group as the difference between day 1 (acute THC treatment) and day 0 (baseline). This difference score was calculated for each individual and then averaged by strain and sex. Difference scores provide a more direct method to investigate drug response and are more robust to the effects of batch [279]. To determine the effect of strain and sex on initial sensitivity to THC for each trait (y), multifactor ANOVA in the form of $\mathrm{y} \sim$ Strain*Sex was performed using base functions in R. Post-hoc analysis to determine pair-wise significance between strains for each sex and time point was performed using Tukey's HSD test (TukeyHSD base function in R).

Rapid tolerance was calculated for the THC group as the difference between day 2 (second THC exposure) and day 1 (acute THC treatment). This difference score was calculated for each individual and then averaged by strain and sex. To determine the effect of strain and sex on rapid tolerance to THC for each trait $(\mathrm{y})$, multifactor ANOVA in the form of $\mathrm{y} \sim$ Strain*Sex was performed using base functions in R. Post-hoc analysis to determine pair-wise significance between strains for each sex and time point was performed using Tukey's HSD test as described above.

\section{Results}

\section{Sex and Strain Differences in Psychomotor Response to THC}

Results are summarized in Figure 2-2. Compared to VEH, treatment with THC $(10 \mathrm{mg} / \mathrm{kg}$, i.p.) resulted in a significant reduction in the time spent mobile in the open field $[\mathrm{F}(1,284)=353.4, \mathrm{p}<0.001]$. There was also a significant main effect of strain [B6 $>\mathrm{D} 2 ; \mathrm{F}(1,284)=88.2, \mathrm{p}<0.001]$, post-injection time [75 $\mathrm{min}>30 \mathrm{~min}>0 \mathrm{~min}$; 

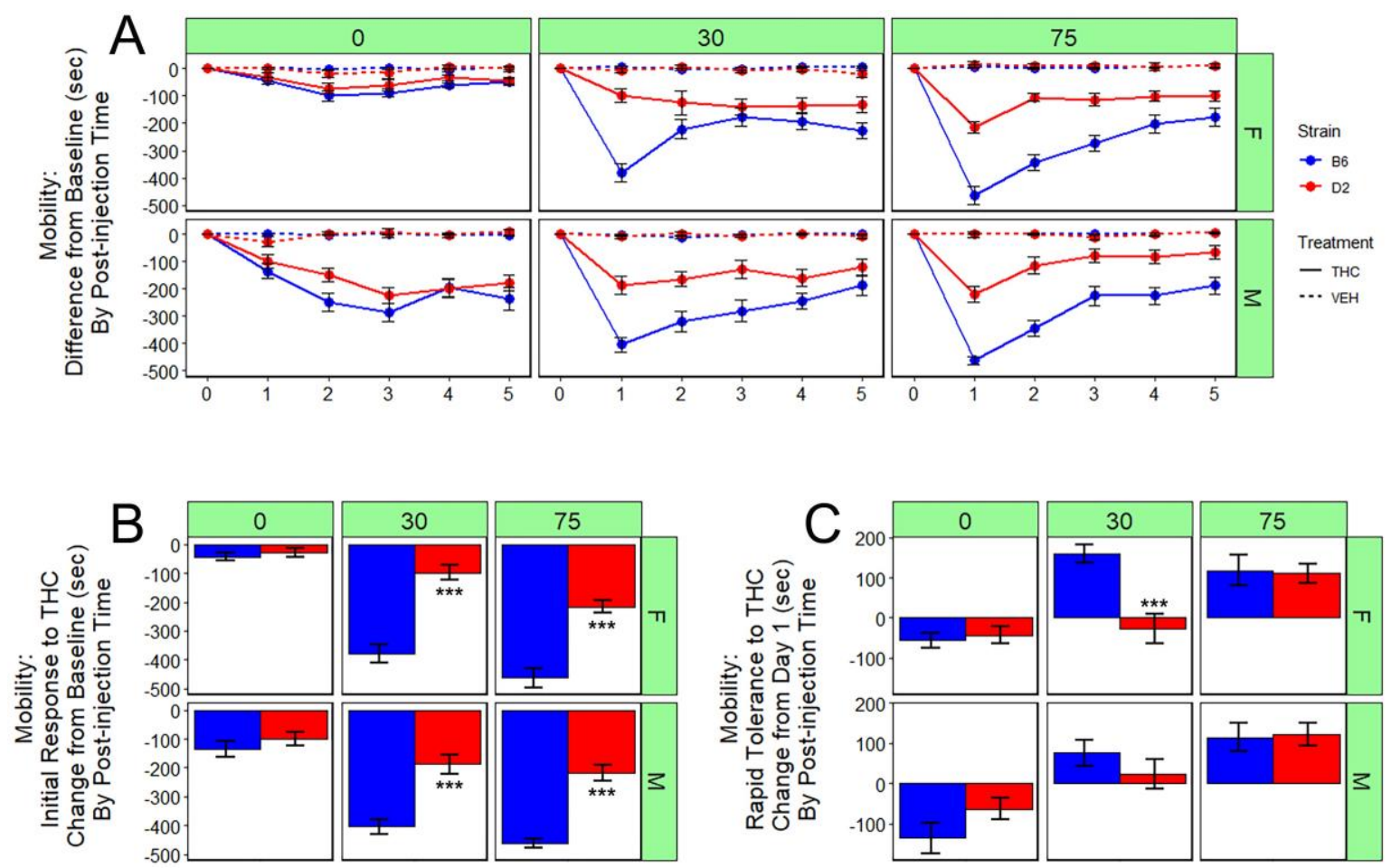

Figure 2-2. Motor response to THC.

(A) Time mobile in seconds (sec) in the open field for B6 and D2 females (top panel) and males (bottom panel) at three time points $(0,30$, or $75 \mathrm{~min})$ post-injection of THC (10 $\mathrm{mg} / \mathrm{kg}$ ) or VEH. Trait data for every individual have been transformed relative to baseline (day 0) by subtracting trait values for each day by the trait values for day 0 and averaging data by strain, sex, treatment, day, and post-injection time. Data represented as mean SEM. Hypolocomotion is evident as early as $0-10 \mathrm{~min}$ (0-min time point) post-injection on day 1 and is more prominent in males. Peak initial response to THC occurs at 75 min post-injection on day 1 . Both males and females of the B6 strain are more sensitive to the locomotor effects of THC relative to both sexes of the D2 strain, and males are more sensitive than females. Rapid tolerance is evident upon second exposure to THC and results in a modest $* 25 \%$ (B6) to $* 50 \%$ (D2) increase in motor activity. However, full desensitization (return to baseline levels) is not observed after five consecutive treatments. (B) Initial response to THC is the difference between day 1 and 0 (baseline) for the THC treatment group (average of each individual's difference score). Significant strain differences in initial sensitivity to the locomotor effects of THC were evident on day 1 for both males and females at the 30 and 75 min post injection time points based on Tukey's HSD test. (C) Rapid tolerance to THC the difference between day 2 and 1 (THC treatment group only). Rapid tolerance to the locomotor effects of THC is not evident at 0 min post-injection. Relative to D2 mice, B6 mice demonstrate significantly greater rapid tolerance to the locomotor effects of THC at $30 \mathrm{~min}$ post-injection. B6 females exhibited significantly greater rapid tolerance relative to D2 females at $30 \mathrm{~min}$ post-injection. Significance defined as $\mathrm{p}<0.05^{*}, \mathrm{p}<0.01^{* *}$, and $\mathrm{p}<0.001^{* * *}$. 
$\mathrm{F}(1,284)=43.6, \mathrm{p}<0.001]$, and $\operatorname{sex}[\mathrm{M}>\mathrm{F} ; \mathrm{F}(1,284)=20.8, \mathrm{p}<0.001]$ on reduction of locomotor activity.

The B6 strain was more sensitive to the motor depressant effects of THC relative to D2 and this difference was enhanced at 30 and 75 min post-injection as indicated by significant interactions between treatment and strain $[\mathrm{F}(1,284)=43.3, \mathrm{p}<0.001]$, strain and post-injection time $[\mathrm{F}(1,284)=32.7, \mathrm{p}<0.001]$, and treatment, strain and time $[\mathrm{F}(1,284)=8, \mathrm{p}<0.01]$. In addition, there were significant interaction between treatment and sex $[\mathrm{F}(1,284)=10.0, \mathrm{p}<0.01]$, time and $\operatorname{sex}[\mathrm{F}(1,284)=29.6, \mathrm{p}<0.001]$, and treatment, time, and sex $[\mathrm{F}(1,284)=29.6, \mathrm{p}<0.001]$, with males exhibiting an earlier (post-injection time 0) and more pronounced response to the motor effects of THC relative to females and vehicle treatment at 30 min post-injection.

Significant strain differences in initial sensitivity to the locomotor effects of THC upon first exposure were observed at both the $30 \min [\mathrm{B} 6>\mathrm{D} 2 ; \mathrm{F}(1,48)=72.7$, $\mathrm{p}<$ $0.001)]$ and the $75 \min [\mathrm{B} 6>\mathrm{D} 2 ; \mathrm{F}(1,78)=92.6, \mathrm{p}<0.001)]$ time point (Figure 2-2B). At both 30 and 75 min post-injection of THC, B6 males and females demonstrated greater initial sensitivity to the locomotor depressing effects of THC relative to D2 males and females (p adjusted $<0.001$; Figure 2-2B).

No significant main effects of sex or interaction effects were observed at these time points. However, a trend for greater initial sensitivity to the locomotor effects of THC in males relative to females was observed at the 30 min time point $[\mathrm{F}(1,48)=72.7$, $\mathrm{p}=0.06)]$. A significant main effect of sex on initial sensitivity to the locomotor effects of THC upon first exposure was observed at 0 min $[\mathrm{M}>\mathrm{F} ; \mathrm{F}(1,78)=14.3$, $\mathrm{p}<0.001]$ post-injection of THC (Figure 2-2B). No significant effect of strain or interaction effects were observed at this early time point.

Rapid tolerance to the locomotor effects of THC following two consecutive treatments (day 2) was evident in males and females of both strains at 30 and 75 min post-injection, but not at 0 min post-injection (Figure 2-2A). A significant main effect of strain $[\mathrm{B} 6>\mathrm{D} 2 ; \mathrm{F}(1,48)=14.6, \mathrm{p}<0.001)]$ and a significant sex-by-strain interaction effect $[\mathrm{F}(1,48)=4.2, \mathrm{p}<0.05)]$ on rapid tolerance upon second exposure to THC was observed at the 30 min post-injection only (Figure 2-2C). Post-hoc analysis of the 30 min post-injection time point revealed that B6 females exhibited significantly greater rapid tolerance to the locomotor effects of THC relative to D2 females (p adjusted < 0.001, Figure 2-2C).

For B6 males and females, rapid tolerance to the locomotor effects of THC upon second exposure accounted for a $\sim 25 \%$ change in the direction of baseline levels of activity at the time of maximal initial response (THC group, 75 min post-injection, (Figure 2-2A). For D2 males and females, rapid tolerance to the locomotor effects of THC upon second exposure accounted for a $\sim 50 \%$ change in the direction of baseline levels of activity at the time of maximal initial response (THC group, 75 min postinjection, (Figure 2-2A). For both strains and sexes, and at every time point post- 
injection, the return to baseline was still incomplete after five consecutive daily exposures to THC (Figure 2-2A).

\section{Sex and Strain Differences in the Hypothermic Response to THC}

There were significant main effects of treatment [THC > VEH; $\mathrm{F}(1,181)=106.4$, $\mathrm{p}<0.001]$, post-injection time [60 $\mathrm{min}>30 \mathrm{~min} ; \mathrm{F}(1,181)=11.6, \mathrm{p}<0.001]$, and $\mathrm{sex}[\mathrm{F}>$ $\mathrm{M} ; \mathrm{F}(1,181)=11.5, \mathrm{p}<0.001]$ on body temperature (Figure 2-3). In addition, a significant time-by-sex interaction effect $[\mathrm{F}(1,181)=3.7, \mathrm{p}=0.05]$ and a non-significant trend for a treatment-by-time interaction effect [60 $\min >30 \mathrm{~min} ; \mathrm{F}(1,181)=3.7, \mathrm{p}=0.05]$ on hypothermic response were detected. Females exhibited the largest response to treatment and the magnitude of the treatment effect on hypothermia was greatest at 60 min post-injection.

There was a significant main effect of sex on initial response to $\operatorname{THC}[\mathrm{F}(1,28)=$ $8.6, \mathrm{p}<0.01]$ at $30 \mathrm{~min}$ post-injection. There were no main effects of strain or sex-bystrain interaction effects on initial response to the hypothermic effects of THC at this time point. At 60 min post-injection of THC there were significant main effects of sex $[\mathrm{F}(1,89)$ $=8.4, \mathrm{p}<0.01]$ and strain $[\mathrm{F}(1,89)=4.0, \mathrm{p}<0.05]$ on hypothermic initial response to THC. At both post-injection time points, females exhibited greater initial sensitivity to the hypothermic effects of THC relative to males (Figure 2-3B). At the 60 min time point, the D2 strain exhibited greater initial sensitivity to the hypothermic effects of THC relative the B6 strain (Figure 2-3B).

At 60 min post-injection (maximum initial response to THC) rapid tolerance to the hypothermic effects of THC upon second exposure accounted for a $50 \%$ or greater change in the direction of baseline body temperature ( $70 \%$ in B6 females, $\sim 50 \%$ in B6 males, $\sim 80 \%$ in D2 females, and $\sim 65 \%$ in D2 males; Figure 2-3A). In contrast to the mobility trait, the return to baseline was complete by the third exposure to THC in both strains, sexes and post-injection time points (Figure 2-3A).

At 30 min post-injection of THC, there was a significant main effect of sex on rapid tolerance to THC-induced hypothermia $[\mathrm{F}(1,28)=12.8$, $\mathrm{p}<0.05)]$. At 60 min postinjection of THC, there was a significant main effect of both $\operatorname{sex}[\mathrm{F}(1,81)=9.6, \mathrm{p}<$ $0.01)]$ and strain $[F(1,81)=4.2, p<0.05)]$ on rapid tolerance to hypothermia. Relative to males, females exhibiting significantly greater rapid tolerance to the hypothermic effects of THC at both time points and the D2 strain exhibited greater rapid tolerance at $60 \mathrm{~min}$ post-injection relative to the B6 strain (Figure 2-3C).

\section{Strain Differences in the Antinociceptive Response to THC}

We observed significant main effects of treatment $[\mathrm{THC}>\mathrm{VEH} ; \mathrm{F}(1,152)=50.6$, $\mathrm{p}<0.001]$ and strain $[\mathrm{D} 2>\mathrm{B} 6 ; \mathrm{F}(1,152)=9.8, \mathrm{p}<0.01]$ on tail flick latency in response to a thermal stimulus (Figure 2-4). The D2 strain exhibited longer tail flick latencies 

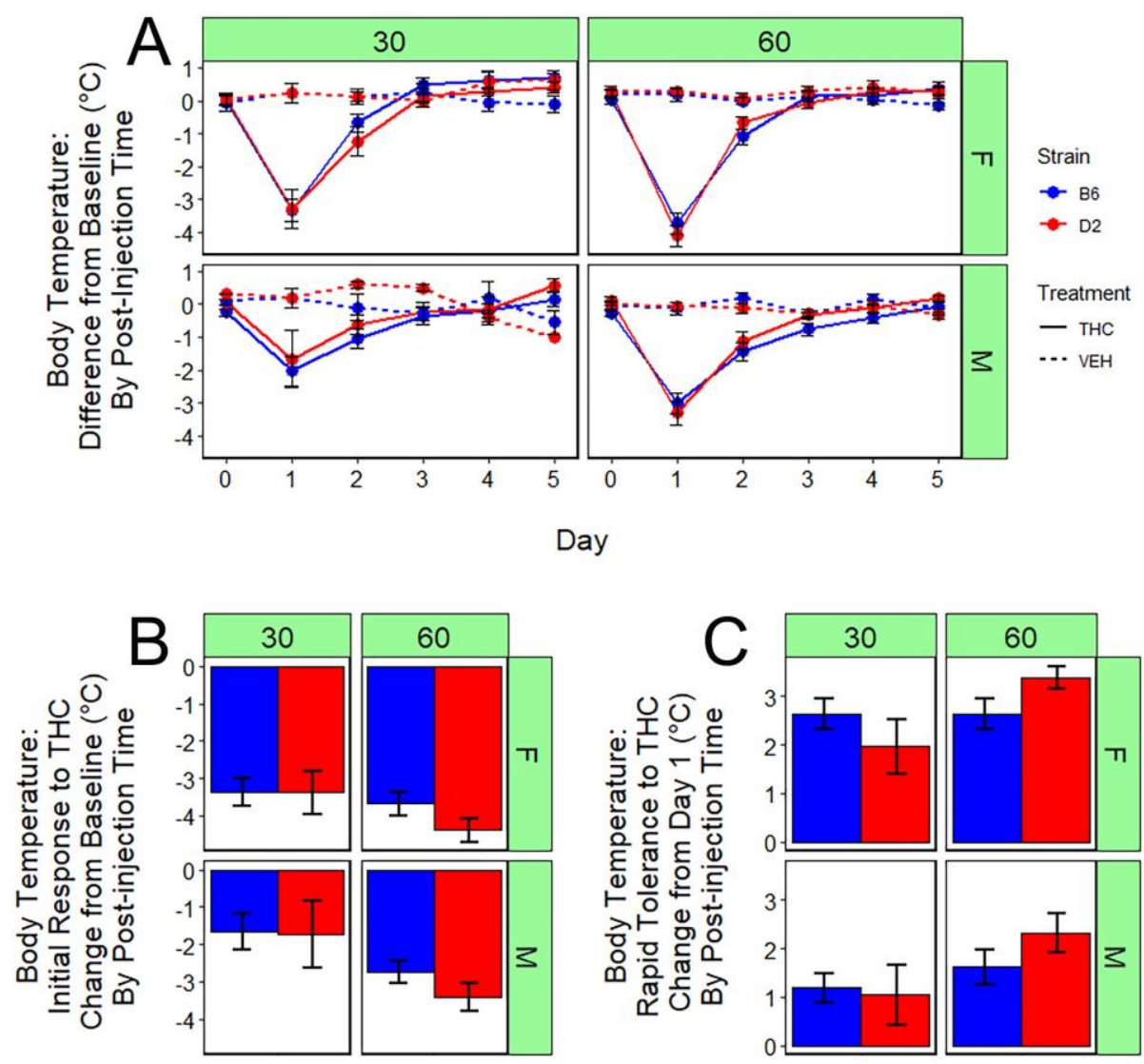

Figure 2-3. Hypothermic response to THC.

(A) Body temperature $\left({ }^{\circ} \mathrm{C}\right)$ for $\mathrm{B} 6$ and D2 females (top panel) and males (bottom panel) at three time points $(0,30$, or $60 \mathrm{~min})$ post-injection of THC $(10 \mathrm{mg} / \mathrm{kg})$ or VEH. Trait data for every individual have been transformed relative to baseline (time 0 ) by subtracting 30- and 60-min trait values for each day by the trait values for time 0 and averaging data by strain, sex, treatment, day, and post-injection time. Data represented as mean - SEM. Profound hyperthermia is evident in both strains at 30 min post-injection on day 1 and is more pronounced in females. Peak initial response to THC occurs at 60 min post-injection. Rapid tolerance upon second exposure results in a substantial increase in body temperature toward baseline in both strains and sexes. Desensitization is complete in both strains and sexed by the third exposure to THC. (B) Initial response to THC is the difference between day 1 and 0 (baseline) for the THC treatment group. Females demonstrate significantly greater hypothermia after a single exposure to THC at 30 and 60 min post-injection relative to males. At the 60 -min time point only, a significant main effect of strain was observed, in which the D2 strain exhibits greater sensitivity to the hypothermic effects of THC relative to the B6 strain. There were no significant interaction effects observed between strain and sex. (C) Rapid desensitization to THC is the difference between day 2 and 1 (THC treatment group only).

Desensitization to the hypothermic effects of THC is evident for both strains, sexes, and post-injection times by the third day of treatment. Females demonstrate significantly greater rapid tolerance to the hypothermic effects of THC at all time points. At the 60min time point, the D2 strain exhibits slightly greater rapid tolerance relative to B6. 


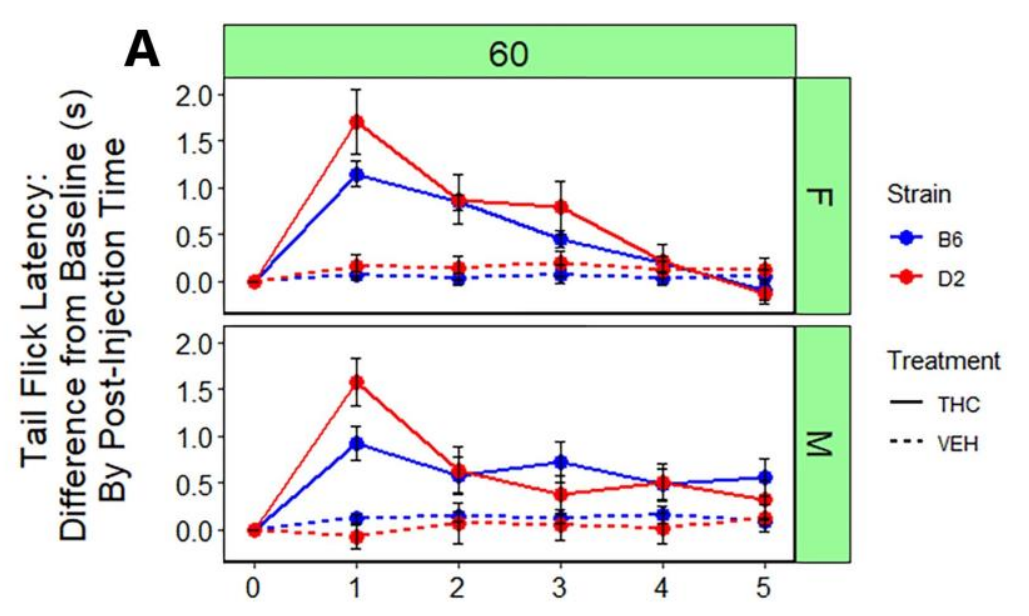

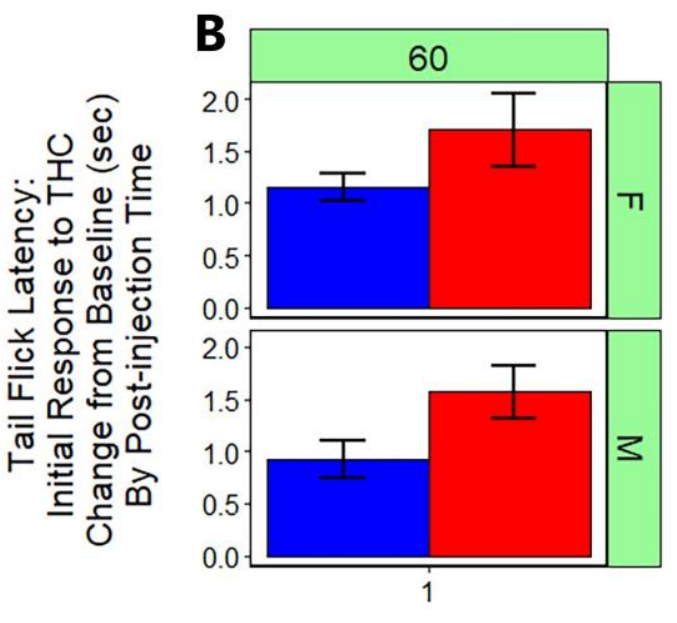

Day 1

Day

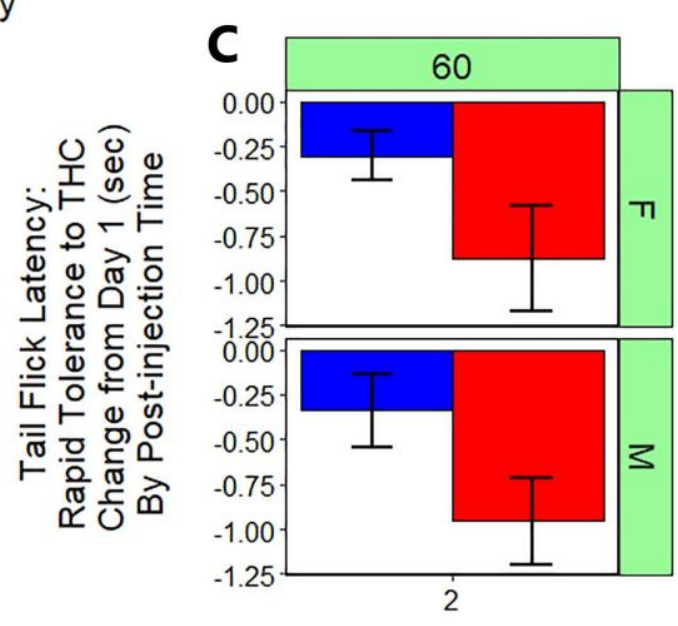

Day 2

Figure 2-4. Antinociceptive response to THC.

(A) Tail withdrawal latency in response to a thermal stimulus for B6 and D2 females (top panel) and males (bottom panel) $60 \mathrm{~min}$ post-injection of THC (10 mg/kg) or VEH. Trait data for every individual have been transformed relative to baseline (day 0 ) by subtracting trait values for each day by the trait values for day 0 and averaging data by strain, sex, treatment, day, and post-injection time. Data represented as mean - SEM. A significant increase in tail flick latency following THC treatment is evident in both strains and sexes. Rapid tolerance upon second exposure is more prominent in the D strain and desensitization is nearly complete in both strains by the fifth treatment, especially in females. (B) Initial response to THC is the difference between day 1 and 0 (baseline) for the THC treatment group (average of every individual's difference score). The D2 strain (both sexes combined) demonstrates significantly greater analgesia after a single exposure to THC relative to the B6 strain. (C) Rapid desensitization to THC is the difference between day 2 and 1 (THC treatment group only). Significant rapid desensitization to the analgesic effects of THC upon second exposure was observed for the B6 strain relative to the D2 strain. 
relative to the B6 strain. There were no significant main effects of sex and there were no significant interaction effects on tail flick latency. There was a significant strain difference in initial response to the antinociceptive effects of THC upon first exposure relative to baseline (Figure $\mathbf{2 - 4 B}$ ) with the D2 strain exhibiting greater sensitivity relative to the B6 strain $[\mathrm{F}(1,107)=6.5, \mathrm{p}<0.05)]$. No significant sex differences in initial response were observed.

For both strains and sexes, rapid tolerance to the antinociceptive of THC upon second exposure accounted for a $\sim 30$ to $60 \%$ change in the direction of baseline (Figure 2-4A). D2 females and males exhibited greater rapid tolerance ( 50 and $60 \%$, respectively) relative to $\mathrm{B} 6$ females and males ( $\sim 30$ and $40 \%$, respectively). The return to baseline was mostly complete in females of both strains by the fourth exposure to THC, however, males of both strains did not completely return to baseline by the fifth exposure (Figure 2-4A). There was a significant main effect of $\operatorname{strain}[\mathrm{F}(1,100)=7.4, \mathrm{p}<0.01$ ) on rapid tolerance to the antinociceptive effects of THC, and D2 mice exhibited greater rapid tolerance to the analgesic effects of THC upon second exposure relative to B6 mice (Figure 2-4C). No significant main effect of sex or sex-strain interaction effects on rapid tolerance were observed.

\section{Discussion}

Here we provide the first comprehensive sex by strain comparison of initial response and rapid tolerance to $\mathrm{THC}$, the main psychoactive component in recreational use cultivars of cannabis. We report strain differences in initial response to the locomotion (B6 > D2) and antinociceptive/analgesic effects (D2 > B6) of THC (10 $\mathrm{mg} / \mathrm{kg}$, i.p.). We also observe strain differences in rapid tolerance to the analgesic effects of THC (D2 > B6) and sex differences in hypothermic responses to THC (F > M).

The response traits measured-locomotion, hypothermia, and antinociceptionare all due to the direct action of THC at CB1R, evidenced by the fact that genetic or pharmacological deletion of CB1R renders mice insensitive to the ability of THC and other CB1R agonists [270-274] to produce these effects. Based on cell-type-specific deletion of CB1R, the cellular mediators of these response traits may be projection neurons in cortex and striatum [134].

This study provides strong support for the hypothesis that genetic variants between B6 and D2 in CB1R signaling pathways, potentially in discrete cortical and/or striatal neuronal populations, may mediate strain differences in initial response and/or rapid tolerance to THC. It is highly likely that preexisting genetic variation between B6 and D2 strains is the main cause of differential sensitivity to the locomotor and analgesic effects of THC upon first exposure. In support of this hypothesis, short-term selective breeding (three generations) for sensitivity or resistance to the initial motor depressant effects of THC in adolescent F2 mice derived from B6 and D2 demonstrated that this trait was indeed moderately heritable and controlled by segregating gene variants between B6 and D2 [280]. 
The precise genetic factors driving differences in THC initial response between B6 and D2 genetic backgrounds is unknown, however, our group has reported preexisting differences in CB1R protein levels in striatum of B6 and D2 [281]. This difference in striatal CB1R levels (B6 > D2) has the potential to directly mediate initial response variation between strains to the motor depressant $(\mathrm{B} 6>\mathrm{D} 2)$, but not the analgesic effects of THC (D2 > B6). However, the relationship between gene variants, CB1R levels, and trait variation is not so straightforward.

The striatum is a key modulator of psychomotor responses to drugs of abuse and CB1R in striatal neurons mediates the psychomotor effects of THC [134]. Consistent with the decreased tolerance to the motor effects of THC, other human and animal studies have also reported higher levels of CB1R following repeated cannabinoid exposure in the striatum relative to cortex and hippocampus [275, 282, 284, 285].

Importantly, there are no variants of predicted high impact in the cognate cannabinoid receptor 1 gene $(C n r l)$, no concomitant change in transcript levels, and no evidence of genetic regulation of transcript expression [281]. Thus, strain differences in striatal CB1R levels are likely the result of genetic variation at other, yet to be identified gene loci involved in regulation of CB1R levels, endogenous cannabinoid levels, and/or signaling pathways.

Tolerance is likely caused by CB1R downregulation (receptor internalization resulting in decreased levels of the receptor) and/or desensitization (modifications of the receptor or signal transduction pathway that decrease signaling) [282, 283]. Relative to the very rapid tolerance observed for the hypothermic effects of THC ( $\sim 3$ days males and females) and the antinociceptive effects of THC ( $\sim 5$ days in females) we observed much less tolerance to the 1 effects of THC in males and females of both strains.

Rapid tolerance following two consecutive exposures to THC was evident for hypothermia and analgesia traits. For both traits, the sex and/or strain with the highest level of initial sensitivity also possessed the highest level of rapid tolerance at matched time points post-injection of THC. This inverse relationship suggests the involvement of the same underlying genetic factors and regulatory pathways. Genetic correlation between traits in populations derived from B6 and D2 or quantification of CB1R levels at baseline and following consecutive THC treatments could be used in future to interrogate the relationship between initial response and rapid tolerance in more detail.

Identification of sex differences in the response to cannabis use and abstinence in humans and cannabinoid response in preclinical models has been hindered by the failure to include an equal representation of female to male subjects in the vast majority of studies [286, 287]. Nevertheless, sex differences in response to cannabis or cannabinoids have been reported in some clinical and preclinical studies [288-292] suggesting that sex, along with genetic factors, could contribute to individual variation in risk of adverse side effects of cannabis use and risk of CUD. 
In this study we have evaluated a balanced number of male and female subjects and report large sex differences primarily for hypothermic responses (both initial sensitivity and rapid tolerance) to THC. Sex differences in rapid tolerance to the hypothermic effects of THC have also been reported in rats. Female Wistar rats displayed more rapid tolerance to inhaled THC (200 mg/mL x 2 daily) relative to males [293] as did female Sprague-Dawley rats (30 mg/kg THC delivered $\mathrm{x} 2$ daily s.c.) relative to males and ovariectomized females [294]. Taken together these studies suggest a possible role for estrogen, at least in the development of tolerance to the hypothermic effects of THC. However, the exact signaling mechanism and underlying circuitry influencing sex differences in response to THC have not been elucidated.

There are several caveats that need to be addressed. First, we were primarily focused on initial response and rapid tolerance to THC. Thus, the phenotyping pipeline explored an abbreviated exposure to THC spanning five daily exposures and does not address the effects of chronic cannabinoid exposure and withdrawal. Second, humans typically consume cannabis in the native state and are therefore exposed to $\sim 100$ constituent cannabinoids whose targets and interactions are still not well understood. Here, we have only profiled the response to a single main psychoactive component, THC. However, further investigation with cannabinoid mixtures is warranted and could help elucidate the molecular mechanisms underlying the response to other cannabinoids, such as CBD, or effects from the combined actions of constituent phytocannabinoids and terpenes.

In conclusion, this study provides strong evidence that genetic factors in the form of segregating variants between B6 and D2 genomes influence response to THC.

Ultimately, forward genetic strategies in mouse populations with divergent behavioral and physiological responses to THC and other cannabis constituent components can be leveraged to identify genes that reduce adverse health consequences and/or maximize therapeutic properties of cannabis or cannabinoid based therapeutics.

Genetic populations derived from B6 and D2, such as the recombinant inbred BXD population, can now be used to identify the enigmatic genetic factors modulating cannabinoid initial sensitivity and rapid tolerance. Derived by crossing the highly genetically and phenotypically divergent B6 and D2 strains followed by inbreeding of the resulting progeny, the BXD family is the largest and best characterized genetic reference population currently available.

This population is segregating for $\sim 6$ million sequence variants, multiple levels of molecular and behavioral data have been quantified for many individual BXD strains, and this family has been used extensively to study genetic variation in traits related to cognitive function [295-301], anxiety [265, 302, 303], schizophrenia [304], and response to drugs of abuse such as ethanol, nicotine, methamphetamine, cocaine, and opioids [264268]. The availability of deep phenome data and marked trait variation in cognitive, sensorimotor, anxiety, and addiction-like behavior makes the BXDs an ideal population for investigating behavioral and physiological responses to THC and other cannabinoids. Future phenotyping efforts quantifying initial sensitivity and rapid tolerance to THC in 
the BXD panel are expected to result in the identification of key endocannabinoid signaling genes mediating the response to THC. 


\section{CHAPTER 3. GENETIC VARIATION IN INITIAL SENSITIVITY TO THC AMONG BXD RECOMBINANT INBRED STRAINS}

\section{Introduction}

Animal genetic reference populations, such as the BXD recombinant inbred (RI) strains derived from crossing B6 and D2 mice used in this study (Figure 3-1), are a valuable resource for studying genetic drivers of an array of phenotypes, including response to and abuse of alcohol and drugs [265, 266, 305-309]. With over 100 BXD lines available [310], this panel of mice is the largest and best characterized genetic reference population available for genetic variation studies. This population has over 6 million segregating sequence variants with roughly 10,000 known recombinations and has been used in a multitude of behavioral studies including cognitive function, anxiety and stress, mental illness, and response to various drugs [265, 267, 298, 299, 311-313]. GeneNetwork (RRID:SCR_002388) collects and makes publically available the data from many of these studies, making it the largest repository of BXD data on the web $[310,314,315]$. This pre-existing, accessible phenome and genetic data on behavior and drug response makes using the BXD panel for this study even more enticing. There are other mouse genetic reference population that exist to help perform systems genetics studies on complex traits, such as the Diversity Outcross [316] and Collaborative Cross [317]; however, the BXD population is the best for a systems level analysis due to mapping power and resolution, genotype density, strain availability, depth of data (genetic to molecular to physiological to behavioral), availability of analysis tools, and cost effectiveness.

System genetic studies often focus on quantitative traits. Quantitative traits have continuous, scalar variation (ex: blood pressure, height, IQ) and are affected by multiple genes and interactions with the environment. To complicate things further sometimes these traits can also be impacted by epistasis or pleiotropy. Due to this, quantitative traits are sometimes referred to as polygenic or complex traits. These traits are often studied through modeling mouse physiological and behavior assays that allow for detection of quantitative trait loci (QTLs) loci for traits that contain at least a modest level of heritability. QTLs are areas of the genome that are at least in part affecting a phenotype. QTL mapping locates these genetic segments to allow for investigation into the genetic basis of quantitative traits and the potential identification of candidate variants and genes. Data from the BXD reference population also allows correlation and comparison analysis among tested traits from within one study and other studies where the BXDs are used and the data is accessible.

Testing a phenotype and then using molecular tools to determine casual genetic mechanisms is known as forward genetics; in contrast to reverse genetics where you manipulate a gene and observe the outcome. A forward genetics study design can be 


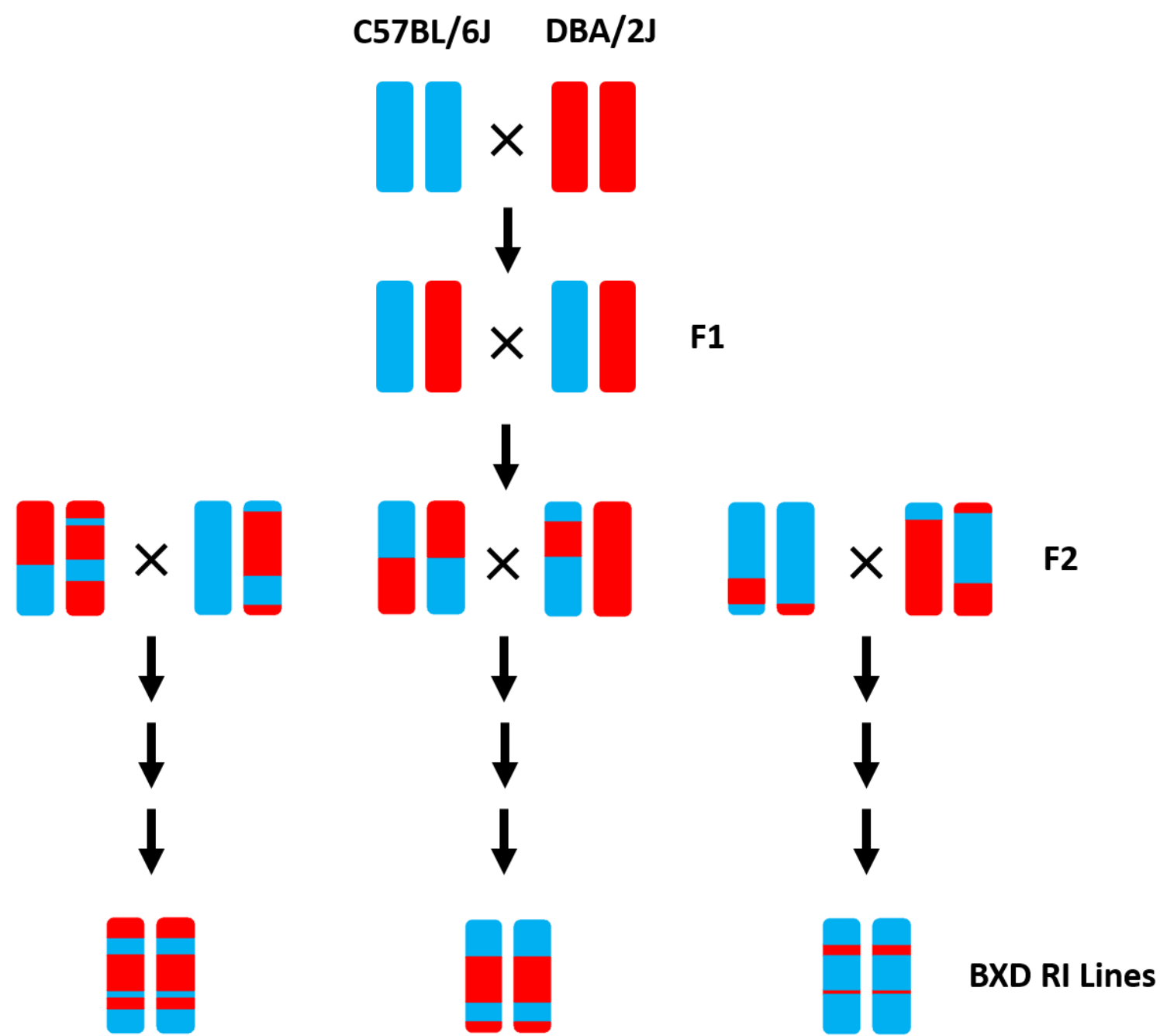

Figure 3-1. Production of recombinant inbred BXD lines.

The BXD mice were generated by first crossing B6 and D2 inbred strains. The F1 mice are then crossed creating genetically diverse, but non-reproducible F2 offspring. The F2 mice are then inbred for 20 or more generations to achieve full homozygosity at every loci. These stable and reproducible recombinant inbred (RI) progeny derived from B6 and D2 are known as the BXDs. 
applied and used to investigate susceptibility and sensitivity to drugs, such as THC. This can potentially help identify individuals who have a pre-disposition for developing dependence and use disorders.

Here we test the same THC response traits seen in Chapter 2 with the parental B6 and D2 strains among a subset of the BXDs. A preliminary number of BXD strains $(\mathrm{N}=20)$ was used in this study. Strain and sex differences were analyzed for each tested trait as well as heritability of the traits. Trait correlation analysis was performed between the BXD THC response traits and existing GN BXD legacy trait data measuring response to drugs of abuse. QTL mapping was performed for all traits. We expect these phenotypic traits to be heritable, with variants in genes between the B6 and D2 parental strains being the driving force of any observed variable response. These genetic mechanisms will be discovered using forward genetic mapping methods with the BXD RI reference population. Genes controlling initial response to THC could also modulate response to other drugs of abuse which will be reflected by correlations between THC response data and legacy trait data from other drugs of abuse in the BXD population.

\section{Materials and Methods}

\section{Experimental Subjects}

BXD mice are maintained in a large breeding colony at the University of Tennessee Health Science Center by the Center for Integrative and Translational Genetics. Mice from this colony were made available upon weaning as part of a pilot project. Each strain was composed of mice from 1 to two litters. The age range of mice on the first day of testing was between 57 to 154 days old. Mice were assigned to testing cohorts consisting of a maximum of 32 mice. Due to the small number of strains included in the pilot project and limited availability in the breeding colony, counterbalancing of strain, sex and condition across cohorts was not possible. At least one week prior to testing, mice were separated into individual housing and handled daily. Handling consisted of lifting mice in either cupped hands (avoiding any lifting by the tail) or a plastic lid from a pipette tip box. Food and water were provided ad libitum and mice were maintained on a $12 \mathrm{~h}: 12 \mathrm{~h}$ light:dark cycle. All testing was performed during the light cycle from 7 am to $4 \mathrm{pm}$. All animal activities were approved by the University of Tennessee Health Science Center Institutional Animal Care and Use Committee.

\section{THC and Vehicle Formulation}

THC was formulated in an ethanol:Cremophor:saline (5:5:90) vehicle followed by filter sterilization. The resulting formulation was stored in the dark and under (4C) refrigeration in a septum sealed vial. Vehicle was prepared in the same manner. THC and vehicle were administered by intraperitoneal (i.p.) injection at a dose of $10 \mathrm{mg} / \mathrm{kg}$ such that a $30 \mathrm{~g}$ mouse received a $100 \mu \mathrm{L}$ injection volume. Each formulation was used 
for the entire testing schedule of each cohort (see details below). Previously we demonstrated that after 8 days, the THC formulation contains $95.2 \%$ of the initial amount of THC [318].

\section{Testing Schedule}

Mice of each strain were randomly assigned to THC ( $\sim 3$ per sex and strain) or vehicle (VEH, 2 per sex and strain) groups, Table 3-1 contains exact numbers. Mice were tested over seven consecutive days. The BXD pipeline mirrored pipeline B from the parental study (Figure 2-1). The first day (Day -1) no injections were given. The second day (Day 0), all mice received an injection of VEH (10 mg/kg). On Days 1 through 5 mice assigned to the THC group received daily injections of $10 \mathrm{mg} / \mathrm{kg}$ THC and mice in the VEH group received daily injections of VEH. On each day, body temperature, latency to withdrawal the tail in response to a thermal stimulus, and activity in the open field were measured at multiple time points in the same animal. Rectal body temperature was measured using a ThermoWorks digital thermometer with a mouse rectal probe adaptor at time 0 , and 60 min post-injection. Tail withdrawal latency was measured 60 min postinjection by gently restraining each mouse in a $50 \mathrm{~mL}$ conical tube. The tail was then submerged $\sim 2 \mathrm{~cm}$ in a $52^{\circ} \mathrm{C}$ water bath. The latency to remove the tail in response to the thermal stimulus was recorded to the nearest second. In most cases, latency to tail flick was measured by two independent experimenters and the resulting scores were averaged. Time spend mobile or immobile (periods of no movement lasting for 3 or more secs) and total distance traveled were measured 30 min post-injection in a $40 \times 40 \times 40 \mathrm{~cm}$ open field over a 10 min interval using video recording and ANY-maze (Stoelting) tracking software.

\section{Initial Response to THC}

Data from day 0 and day 1 were included in the analysis. Individual data points were removed for technical reasons only (e.g. equipment failure during recording). Day 0 and day 1 were analyzed independently to look for any significant interactions on testing days. Initial response was calculated for each group (VEH or THC) as the difference between day 1 and day 0 (baseline). This difference score was calculated for each individual and then averaged by strain and sex. Difference scores provide a more direct method to investigate drug response and are more robust to the effects of batch (note that counterbalancing was not possible in the pilot study design). To determine the effect of strain and sex on initial response to THC for each trait (y), multifactor ANOVA in the form of $\mathrm{y} \sim$ Strain*Sex was performed using base functions in $\mathrm{R}$. 
Table 3-1. Summary of BXD strains.

\begin{tabular}{|c|c|c|c|}
\hline Strain & Sex & THC & Vehicle \\
\hline \multirow{2}{*}{ BXD 1} & Male & 2 & 1 \\
\hline & Female & 0 & 0 \\
\hline \multirow{2}{*}{ BXD 11} & Male & 1 & 1 \\
\hline & Female & 0 & 0 \\
\hline \multirow{2}{*}{ BXD 14} & Male & 5 & 4 \\
\hline & Female & 3 & 2 \\
\hline \multirow{2}{*}{ BXD 15} & Male & 3 & 3 \\
\hline & Female & 0 & 0 \\
\hline \multirow{2}{*}{ BXD 16} & Male & 3 & 2 \\
\hline & Female & 3 & 2 \\
\hline \multirow{2}{*}{ BXD 43} & Male & 2 & 2 \\
\hline & Female & 5 & 4 \\
\hline \multirow{2}{*}{ BXD 44} & Male & 3 & 2 \\
\hline & Female & 3 & 3 \\
\hline \multirow{2}{*}{ BXD 48a } & Male & 4 & 3 \\
\hline & Female & 3 & 2 \\
\hline \multirow{2}{*}{ BXD 62} & Male & 3 & 2 \\
\hline & Female & 3 & 2 \\
\hline \multirow{2}{*}{ BXD 66} & Male & 2 & 2 \\
\hline & Female & 3 & 2 \\
\hline \multirow{2}{*}{ BXD 71} & Male & 2 & 2 \\
\hline & Female & 2 & 3 \\
\hline \multirow{2}{*}{ BXD 73} & Male & 3 & 3 \\
\hline & Female & 3 & 3 \\
\hline \multirow{2}{*}{ BXD 73a } & Male & 3 & 3 \\
\hline & Female & 3 & 2 \\
\hline \multirow{2}{*}{ BXD 74} & Male & 3 & 2 \\
\hline & Female & 3 & 2 \\
\hline \multirow{2}{*}{ BXD 75} & Male & 3 & 2 \\
\hline & Female & 3 & 2 \\
\hline \multirow{2}{*}{ BXD 77} & Male & 3 & 2 \\
\hline & Female & 3 & 2 \\
\hline \multirow{2}{*}{ BXD 83} & Male & 3 & 2 \\
\hline & Female & 2 & 2 \\
\hline \multirow{2}{*}{ BXD 87} & Male & 3 & 2 \\
\hline & Female & 3 & 2 \\
\hline \multirow{2}{*}{ BXD 100} & Male & 3 & 2 \\
\hline & Female & 3 & 2 \\
\hline \multirow{2}{*}{ BXD 101} & Male & 3 & 2 \\
\hline & Female & 4 & 2 \\
\hline
\end{tabular}




\section{Statistical Analysis of THC Response}

Data from day 0 (VEH treatment) and day 1 (THC treatment) were used in a three between-subject (treatment*strain*sex) analysis of variance. The analysis was performed using base functions in $\mathrm{R}$ (anova.lm function). Response during habituation on day -1 was not analyzed. Individual BXD data points were only removed for technical reasons (e.g. equipment failure during recording). Initial response to THC was calculated as the difference between day 1 and day 0 (baseline). This difference score was calculated for each individual and then averaged by strain and sex. Difference scores provide a more direct method to investigate drug response and are more robust to the effects of batch (note that counterbalancing was not possible in the study design). Individual B6 and D2 initial response data points were excluded from further analysis if they were found to be two standard deviations above or below the mean values calculated within strain and sex. The low number of replicates among BXD strains precluded outlier analysis (Table 3-1). To determine the effect of strain and sex on initial response to THC for each trait (y), multifactor ANOVA in the form of $\mathrm{y} \sim$ Strain*Sex was performed using base functions in $\mathrm{R}$ (anova.Im function). Strain averaged sensitivity traits for mobility and analgesia (Record IDs 21481 and 21483, respectively) have been uploaded to the GN resource. A trend towards an interaction between condition and sex was detected for the hypothermia trait. Thus, strain averaged hypothermia trait values were separated by sex and uploaded to the GN resource (Record IDs 21485 and 21487).

\section{Heritability}

Heritability $(\mathrm{H} 2)$ is an estimate of the proportion of the trait variation that can be explained by genetic factors. Broad sense $\mathrm{H} 2$ was estimated for each sex using two different methods based on the variance components of a one-way ANOVA (y Strain). First, H2 was calculated by comparing the between-strain variance with the total variance or between- and within-strain or environmental variance [319]. This can be calculated from an ANOVA results table by dividing the between-strain sum of squares (SS strain) term by the sum of the between-strain and within-strain (total) SS terms [320]. Second, $\mathrm{H} 2$ was estimated using the Intraclass Correlation Coefficient (ICCest function in the ICC package in R) to determine how closely individuals within the same strain resemble each other.

\section{Genetic Mapping}

Two different methods were applied to compute quantitative trait loci (QTL) probability given strain genotypes and initial responses to THC using the GeneNetwork (GN) web service [RRID:SCR_002388]. The first method applied was traditional simple regression (Haley-Knott or $\mathrm{HK}$ ) and the second was genome-wide efficient mixed model association (GEMMA) [321] with the "leave one chromosome out" (LOCO) option. Prior to performing genome scans, each trait distribution was checked for normality using the Probability Plot function in GN. All raw trait data were approximately normally 
distributed and used for QTL mapping as quantile normalization did not impact QTL scores. For both HK and GEMMA, a dense panel of 7,321 markers was used for mapping. QTL were considered noteworthy if they reached a genome-wide suggestive level ( $\mathrm{p}<0.63$, equivalent to a $63 \%$ probability of a false positive or one false positive per genome scan) following permutation (1000 tests) using the HK method and overlapped the peak marker association $[-\log (\mathrm{p})>3]$ detected using GEMMA. At the time of writing permutation was not yet enabled in GN for GEMMA and was performed separately for each as described in [322]. For trait 21481, the 95th percentile (significant) threshold and 67th percentile (suggestive) threshold was 4.8 and 3.8 LOD, respectively. For trait 21483, the 95th percentile (significant) threshold and 67th percentile (suggestive) threshold was 4.5 and 3.6 LOD, respectively. For trait 21485, the 95th percentile (significant) threshold and 67th percentile (suggestive) threshold was 4.7 and 3.7 LOD, respectively. For trait 21487, the 95th percentile (significant) threshold and 67th percentile (suggestive) threshold was 4.7 and 3.7 LOD, respectively.

It should be noted that QTL scans performed on smaller subsets of BXDs, as in our study of 20 strains, are expected to result in identification of suggestive QTLs of large effect. Smaller effect QTL may go undetected when the number of strains in the mapping population is low. For traits demonstrating a normal distribution, it is assumed that additional phenotyping in a larger number of randomly selected individuals from the population will boost the significance level of large effect QTL and result in the detection of additional smaller effect QTL. For example, a mapping population comprised of 20 BXD strains with 4 replicates per strain is only powered to detect a QTL explaining 50\% of the trait variance whereas a population size of $\sim 80$ strains with 4 replicates per strain is well powered to detect a QTL explaining $20 \%$ of the trait variation at $80 \%$ power [323].

\section{Candidate Gene Search}

A 1.5-LOD drop from the peak marker (HK mapping) was used to define an approximate $\sim 95 \%$ confidence interval (CI) for a QTL of interest [324]. For each QTL CI, a complete list of mouse reference genes was generated using the UCSC Genome Browser (RRID:SCR_005780) Table Browser Tool (Group: Genes and Gene Predictions; Track: NCBI RefSeq; Genome: GRCm38/mm10). Genes in the CI were then prioritized based on several criterion. First, all variants distinguishing B6 and D2 within each QTL CI were identified using Sanger's Mouse Genome Project Mouse_SNPViewer/rel-15050 (RRID:SCR_011784). Specifically, SNPs, InDels, and structural variants (SVs) distinguishing B6 and D2 and their predicted consequences were retrieved and compared (by gene symbol) to reference genes located within the boundaries of each QTL CI. Next, legacy mRNA datasets available in GN for BXD brain tissue were queried to identify genes located in each QTL CI whose expression was modulated by variants located within or near the location of the gene itself - a cis expression QTL (eQTL). Brain region data sets (cortex, hippocampus, hypothalamus, nucleus accumbens, striatum) were selected based on regulation by cannabinoid receptor signaling, CB1R expression, and the potential to influence our initial response traits. Datasets included: Hippocampus Consortium M430v2 (Jun06) [GN Accession: GN110 and GEO Series: GSE84767]; 
INIA Hypothalamus Affy MoGene 1.0 ST (Nov10) [GN Accession: GN281 and GEO Series: GSE36674]; HQF BXD Neocortex ILM6v1.1 (Feb08) RankInv [GN Accession: GN284]; VCU BXD NA Et vs Sal M430 2.0 (Oct07) [GN Accession: GN156]; VCU BXD PFC Sal M430 2.0 (Dec06) RMA [GN Accession: GN135; GEO Series: GSE28515]; HQF Striatum Affy Mouse Exon 1.0ST Gene Level (Dec09) RMA [GN Accession: GN399]. Probes that overlap polymorphic SNPs between B6 and D2 and have cis eQTLs with higher expression associated with the B6 allele (ILM6v1.1 and M430 data sets only) are flagged in this table as expression measurements could be biased in the direction of B6 due to technical probe hybridization artifacts. Finally, a search for relevant biological function (i.e. endocannabinoid/cannabinoid receptor signaling) was conducted using the RatsPub web service (RRID:SCR_018905) to search through PubMed (RRID:SCR_004846) literature abstracts for associations between cannabinoidrelated terms and gene symbols for each QTL CI. A secondary search was performed for high priority candidate genes using the RatsPub web service in order to place these genes in a broader context of addiction and psychiatric diseases.

\section{BXD Exploratory Phenome Analysis}

Two recent studies quantified initial response to multiple drugs of abuse (cocaine, alcohol, morphine) or intravenous cocaine self-administration among BXD RI strains $[325,326]$. Both studies also included large numbers of BXD strains, including at least 12 of the strains used to generate our THC initial response data. Associated drug response traits from these studies have been deposited into the GN BXD Published Phenotypes Database. For these reasons, we selected BXD drug response and cocaine selfadministration traits from these studies for comparison with THC initial response traits generate in our study. Trait data (averaged by strain and sex) was obtained with the Get Any search function in GN using the following input: 2681503 19958391(PubMed IDs).

This resulted in 790 traits. The BXD legacy traits mined from GN were further filtered to include traits whose descriptions included the terms cocaine, ethanol, MDMA, or morphine and DID NOT include the terms baseline control, control for MDMA, novel open field behavior, or saline control response. Finally, traits were retained for correlation analysis if there were at least 12 matched BXD strains shared between BXD legacy trait data and the THC initial response traits. A total of 368 traits (excluding the four initial THC response traits) were included for correlation analysis. Traits were assigned to general categories based on phenotype and whether or not the trait was measured in females, males, or both. The Pearson correlation coefficient and corresponding $\mathrm{p}$-values were calculated for each of the four THC initial response traits and all 314 BXD drug response traits. Significant correlations were associated with a pvalue $<0.05$. 


\section{Results}

\section{Variation in Initial Response to THC among BXDs}

Activation of CB1R in discrete neuronal populations (Monory et al. 2007) by THC results in a well characterized trait spectrum that includes a reduction in spontaneous locomotion, hypothermia, and analgesia. Genetic or pharmacological deletion of CB1R abrogates these effects (Compton et al. 1996; Huestis et al. 2001; Ledent et al. 1999; Rinaldi-Carmona et al. 1994; Zimmer et al. 1999). Thus, quantification of mobility, hypothermia, and analgesia following THC exposure is a highly specific method to measure response to THC mediated through CB1R signaling pathways. In a previous study, we identified strain and sex differences in mobility, hypothermia, and analgesia following THC exposure $(10 \mathrm{mg} / \mathrm{kg})$ between the parents of the BXD family - B6 and D2 (Parks et al. 2020). Here we test the hypothesis that polymorphic variants segregating among $\mathrm{B} 6, \mathrm{D} 2$, and their BXD RI progeny cause differences in the initial response to THC. If response to THC is variable among BXD strains and can be attributed to heritable genetic factors, forward genetic mapping in the $\mathrm{BXD}$ population has the potential to identify variants in effectors of CB1R signaling causing this variation.

Treatment (i.e. baseline values on day 0 compared to day 1 values following THC treatment) had a significant (all $p$-values $<0.001$ ) main effect on the expression of all three initial response traits in our BXD cohort. Relative to the VEH treatment on day 0 , treatment with THC on day 1 reduced time spent mobile in the open field $(\mathrm{F} 1,220=$ $743.11, \mathrm{p}<0.001)$, decreased body temperature $(\mathrm{F} 1,232=588.27, \mathrm{p}<0.001)$, and increased the latency to withdraw the tail in response to a thermal stimulus $(\mathrm{F} 1,230=$ $141.34, \mathrm{p}<0.001)$. Strain also contributed significantly to response variation for mobility $(\mathrm{F} 21,220=7.74, \mathrm{p}<0.001)$, hypothermia $(\mathrm{F} 21,232=3.0, \mathrm{p}<0.01)$, and analgesia $(\mathrm{F} 21,230=2.89, \mathrm{p}<0.001)$. A significant main effect of sex was observed for hypothermia $(\mathrm{F} 1,232=11.07, \mathrm{p}<0.01)$ and analgesia $(\mathrm{F} 1,230=7.24, \mathrm{p}<0.01)$. Importantly, a significant condition-by-strain interaction effect was observed for mobility $(\mathrm{F} 21,220=7.40, \mathrm{p}<0.001)$ and hypothermia $(\mathrm{F} 21,232=2.81, \mathrm{p}<0.001)$. A significant sex-by-condition interaction was also observed for the hypothermia trait $(\mathrm{F} 1,232=6.81$, $\mathrm{p}<0.01)$.

Initial responses (differences from baseline) to THC measured in 20 BXD strains are summarized in Figure 3-2. There was a significant effect of strain on initial response to THC for the mobility $(\mathrm{F} 21,102=9.03, \mathrm{p}<0.001)$ and hypothermia $(\mathrm{F} 21,112=2.73$, $\mathrm{p}$ $<0.001)$ traits. There was a significant main effect of $\operatorname{sex}(\mathrm{F} 1,112=8.14, \mathrm{p}<0.01)$ and a trend towards a sex-by-strain interaction effect $(\mathrm{F} 12,112=1.58, \mathrm{p}=0.08)$ on initial response to THC for hypothermia. No main effect or interaction effect of sex was observed for initial response to THC on mobility. No significant main effects of strain or sex on initial response to THC were detected for analgesia. 
Figure 3-2. Initial response to THC in the recombinant inbred BXD strains.

There is wide phenotypic variation in both sex and strain to the initial effects of THC (10 $\mathrm{mg} / \mathrm{kg}$ ) among the BXD population. Strains are on the $\mathrm{X}$-axis and initial response (difference between response on day 1 and response on day 0 ) is on the y-axis. Negative values indicate a decrease in response on Day 1 compared to Day 0 and positive values indicate an increase. Parental strains are indicated in blue (B6) and red (D2) and BXD strains are in numerical order by strain number in alternating black and gray. (A) There is a significant $(\mathrm{p}<0.001)$ effect of condition, strain, and condition-by-strain on initial response to the motor depressing effects of THC as measured by time mobile in the open field 30 min post-injection. B6 and BXD strains 16 and 87 have greater motor depression following exposure to THC and D2 and BXD strains 71 and 83 have less sensitivity. (B) The antinociceptive effects of THC also had a significant effect of condition $(p<0.001)$ and strain $(\mathrm{p}<0.05)$ as measured by tail flick withdrawal latency at 60 minutes postinjection. D2 and BXD strains 14, 15, and 73a have greater sensitivity to the analgesic effects of THC while BXD strains 75, 77 and 87 have less sensitivity. (C) There was main significant effect of condition ( $<$ < 0.001), strain $(\mathrm{p}<0.001)$ and sex $(\mathrm{p}<.05)$ to the initial response of hypothermic effects of THC and significant interactive effects of condition-by-strain $(\mathrm{p}<0.001)$ and condition-by-sex $(\mathrm{p}<0.05)$. Body temperature was measure 60 minutes post-injection. Female BXD strains 62, 71, and 101 and male BXD strains 16, 77, and 87 have greater sensitivity to the hypothermia inducing effects of THC while female BXD strains 83 and 43 and male BXD strains 11, 48a, and 74 have less sensitivity. 


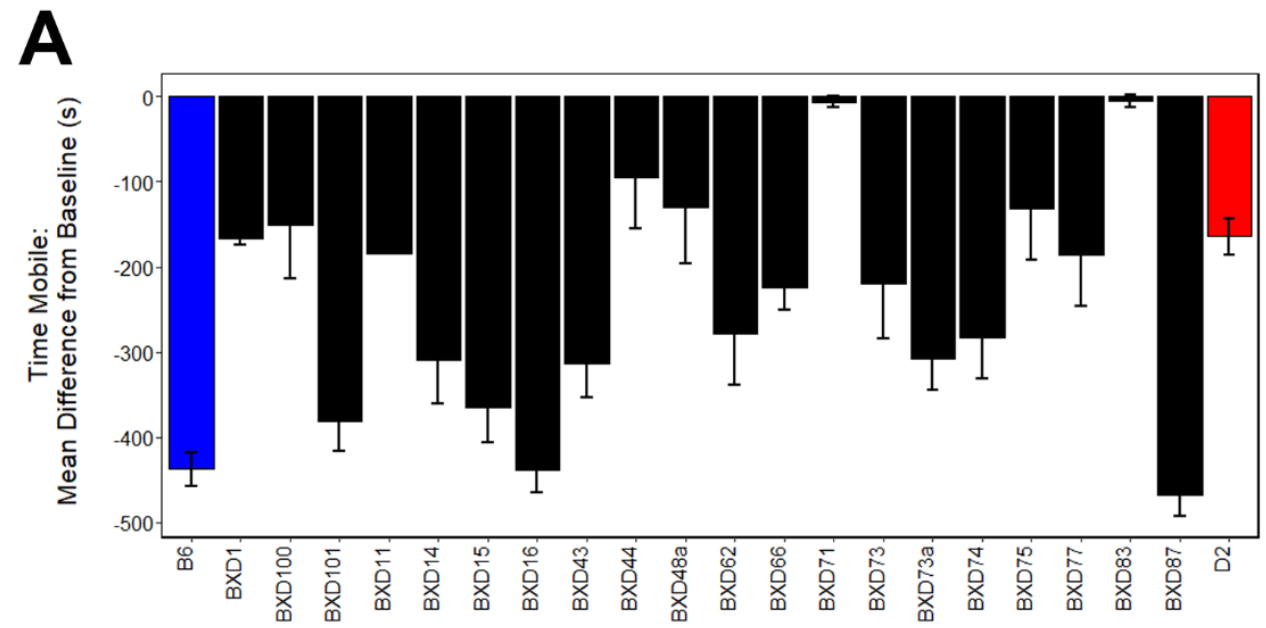

B

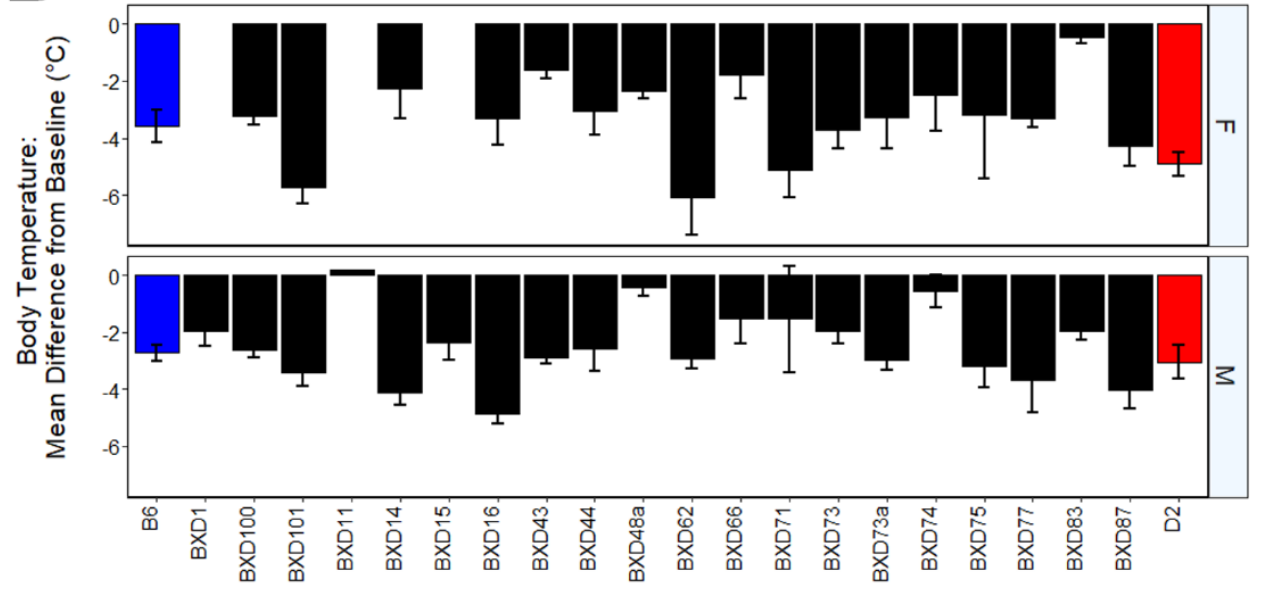

C

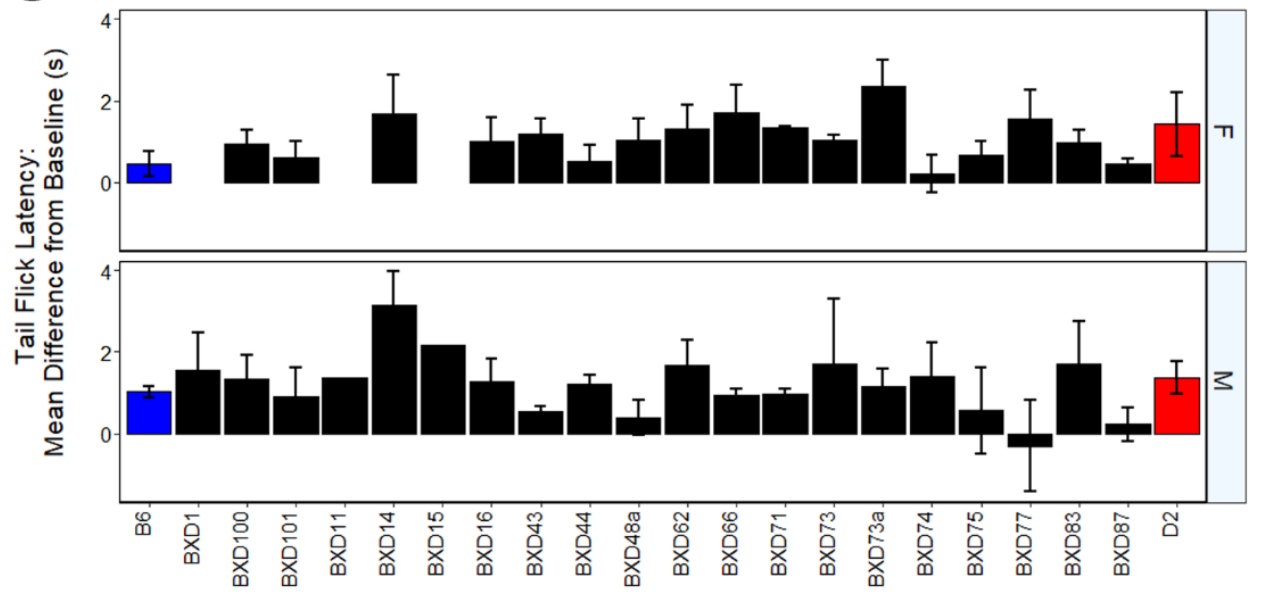


Two different methods ( $\mathrm{H} 2$ and the ICC) based on the variance components of a one-way ANOVA were used to estimate heritability - the contribution of genetic factors to trait variation - in initial responses to THC among our subset of BXD strains (Table 3-2). In general, THC response traits demonstrated high heritability. Tail withdrawal latency (a measure of analgesia) was an exception. Higher heritability was observed for the mobility and hypothermia traits, which indicated a strong contribution of genetic factors to phenotypic variation. Mobility and hypothermia traits also demonstrated sexual dimorphism with regards to heritability. Time mobile had higher heritability in males whereas hypothermia had higher heritability in females. The overall initial response distribution for each trait within males and females of each strain can be found in Figure 3-3.

\section{Initial Response to Mobility and Hypothermia Influenced by Chr 11}

Examination of correlation structure can provide evidence of underlying coregulation of trait expression due to genetic, biological, or environmental factors. We observed a significant $(\mathrm{p}<0.01)$ and positive correlation between time mobile (males and females combined) and hypothermia (males only) following the first exposure to THC in our cohort of 20 BXD strains (Figure 3-4). Note that the hypothermia trait was separated into male and female responses because each sex responds differently to THC (i.e. there was a significant condition-by-sex interaction effect). Hypothermic response to THC in males and females was modestly and positively correlated (but not significant). This was also observed for the hypothermic response in females and time mobile following THC treatment. In contrast, THC-induced analgesia was uncorrelated with the mobility and hypothermia traits.

Our subset of $20 \mathrm{BXD}$ strains was underpowered for mapping small effect QTL. However, heritability estimates were high for several initial THC response traits (i.e. change in time mobile in the open field in response to THC), indicating strong regulation of trait expression by genetic factors. To explore the potential for future genetic mapping studies of these traits in a larger BXD panel, we performed QTL scans for all traits. As expected, no genome-wide significant QTLs (LOD > 3.5) were detected for any trait (Figure 3-4B) using traditional interval mapping (HK) methods. However, suggestive QTLs (LOD > 2.5) were detected for all THC initial response traits (Table 3-3).

Moreover, the highly correlated mobility and male hypothermia traits were co-regulated by a QTL on Chr 11 with a 1.5 LOD drop confidence interval (CI) between 80 and 90 $\mathrm{Mb}$ (Figure 3-4B).

Shared genetic co-regulation of mobility and male hypothermia traits on Chr 11 was also replicated using a different QTL mapping method (GEMMA) that leverages linear mixed-models and accounts for population family structure (Figure 3-4C). Suggestive QTLs for initial response to the hypothermic (Chr 19 in males and Chr 13 in females) and analgesic (Chrs 1 and 4) effects of THC were also replicated across both QTL mapping methods. However, the mobility (Chr 11) and analgesia QTL (Chr4) did not meet the suggestive threshold using GEMMA. Additional suggestive QTLs were 
Table 3-2. Estimates of heritability among BXD and parental strains.

\begin{tabular}{lcccc}
\hline IS Trait & H2 $(\mathbf{F})$ & H2 $(\mathbf{M})$ & ICC F & ICCM \\
\hline Time Mobile & 0.70 & 0.75 & 0.52 & 0.58 \\
Hypothermia & 0.57 & 0.47 & 0.33 & 0.17 \\
Analgesia & 0.30 & 0.35 & -0.05 & 0.02 \\
\hline
\end{tabular}

Notes: IS = initial sensitivity; $\mathrm{H} 2$ = broad sense heritability; ICC = intraclass coeffient estimate of heritability; $\mathrm{F}=$ female; $\mathrm{M}=$ male.
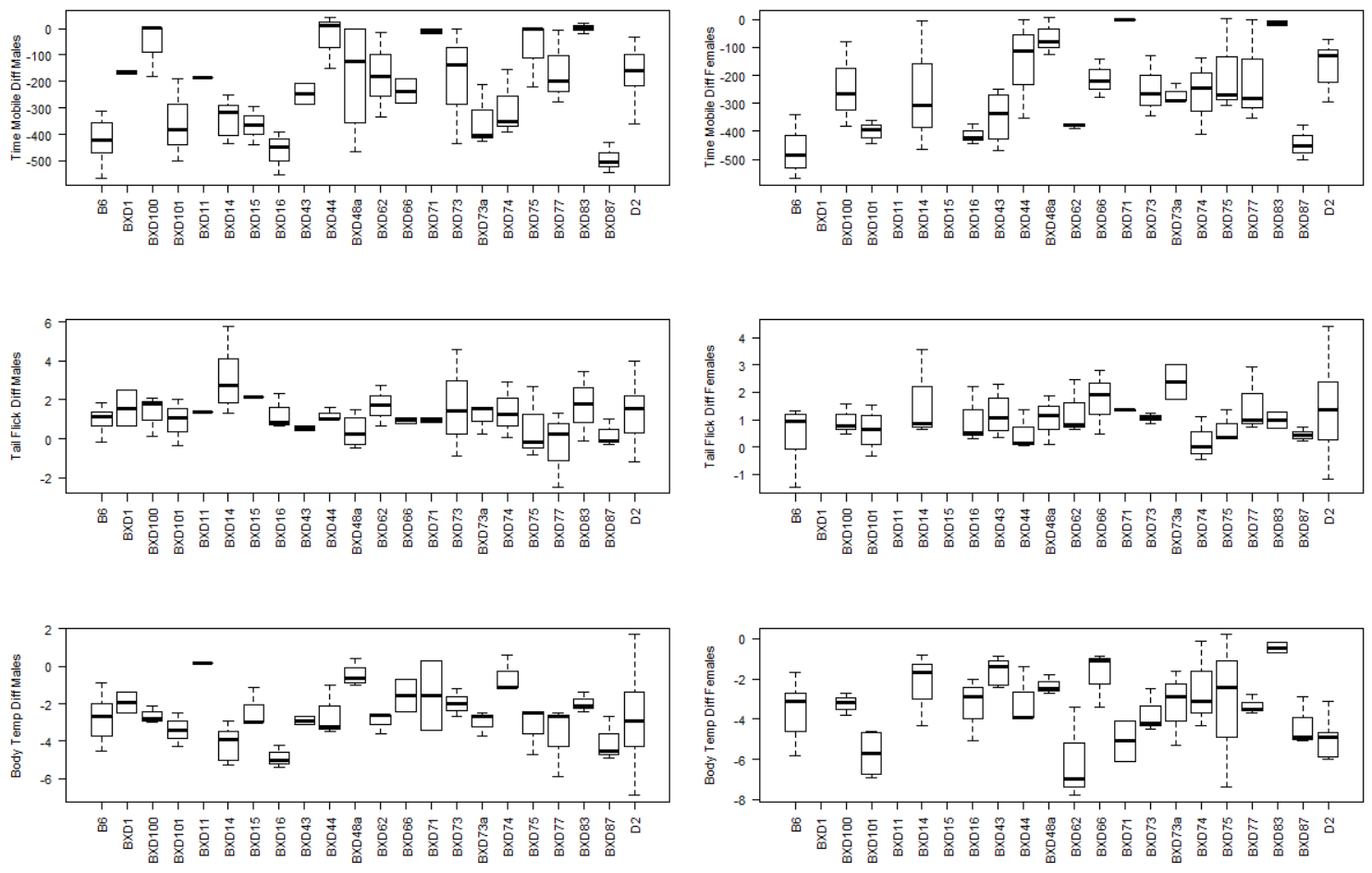

Figure 3-3. Distribution of initial response traits.

Strains are shown on the $\mathrm{X}$-axis and the $\mathrm{Y}$-axis shows response (difference between baseline day 0 and day 1). Male and female responses for each trait are shown in the left and right columns, respectively. 

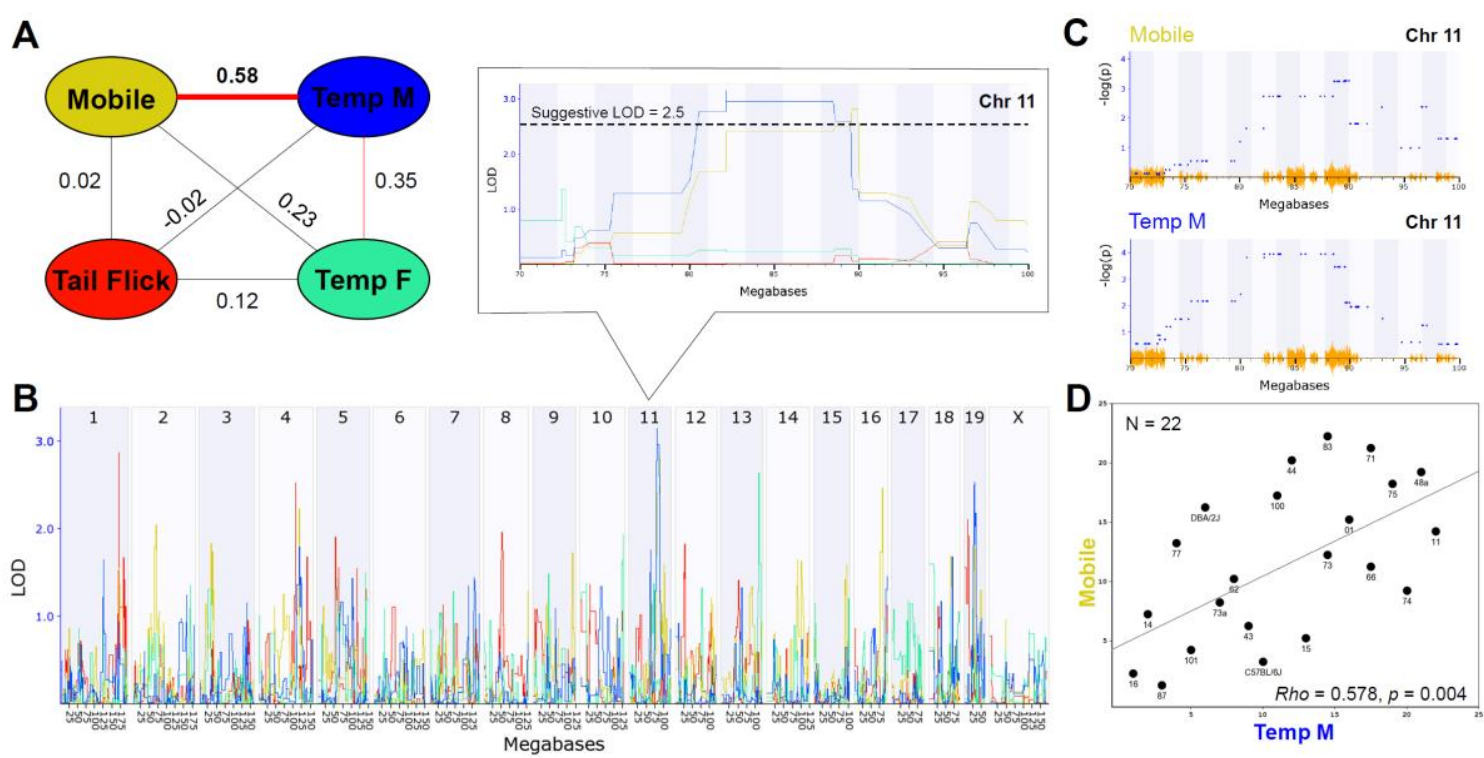

Figure 3-4. THC initial response trait correlations and genetic co-regulation.

Trait correlation network constructed after calculating Pearson's correlation on ranked trait values to generate Spearman's rank order correlation coefficient (Rho or $P$ ). The hypothermia trait was split by sex due to a condition-by-sex interaction effect on body temperature change in response to THC. Changes in time mobile in the open field and body temperature in males following a single THC $(10 \mathrm{mg} / \mathrm{kg})$ exposure are significantly $(\mathrm{p}<0.01)$ correlated. Weak (not significant) correlations are also observed between immobility and hypothermia in females following a single THC exposure and between changes in male and female hypothermia in response to THC. (B) Genetic regulation of THC initial response traits is observed, albeit at a suggestive level with no traits passing the threshold for genome-wide significance $(p<0.05)$. Interval maps for each trait shown as a different line color corresponding to the correlation network in (A). The strength of association (LOD) on the $\mathrm{Y}$-axis is plotted for each trait across the genome (megabase position on each chromosome or Chr) on the $\mathrm{X}$-axis. Change in mobility and male body temperature are regulated by the same suggestive locus on Chr 11 (zoomed region in boxed area). (C) Use of an alternative linear mixed-model QTL mapping method (GEMMA, see methods) resulted in replication of genetic co-regulation of initial motor and hypothermia THC response traits from the same Chr 11 interval. Strength of association or $-\log (\mathrm{P})$ values ( $\mathrm{Y}$-axis) shown for each marker (blue dots) within the Chr 11 QTL interval for mobility and temperature (males) initial THC response traits. Mapping with linear mixed-models can account for population family structure or kinship within the BXD population that is not addressed using traditional interval mapping. SNP density plotted in orange on the X-axis. (D) Scatterplot describing the relationship between average change in time mobile in the open field in response to THC (X-axis) and average change in male body temperature in response to THC (Y-axis) for each BXD strain based on Pearson's correlation on ranked trait data. 
Table 3-3. Summary of suggestive QTLs for THC initial response traits.

\begin{tabular}{lcccc}
\hline Trait & LOD & Chr & 1.5 LOD CI (Mb) & GEMMA-log $(\boldsymbol{p})($ Peak Chr:Mb) \\
\hline Time Mobile & $2.8^{\#}$ & 11 & $80,036,498-90,077,597$ & $3.2(\operatorname{Chr} 11: 89,612,498)$ \\
Hypothermia & $3.2^{\#}$ & 11 & $80,036,498-89,612,498$ & $3.9^{\#}(\operatorname{Chr} 11: 82,187,018)$ \\
(M) & $2.5^{\#}$ & 19 & $27,531,578-36,395,869$ & $3.7^{\#}(\operatorname{Chr} 19: 32,887,054)$ \\
Hypothermia (F) & $2.6^{\#}$ & 13 & $107,382,731-115,929,261$ & $3.7^{\#}(\operatorname{Chr} 13: 110,449,36)$ \\
Analgesia & $2.9^{\#}$ & 1 & $168,144,357-172,235,364$ & $3.9^{\#}(\mathrm{Chr} 1: 170,949,421)$ \\
& $2.5^{\#}$ & 4 & $102,851,020-109,169,766$ & $3.3(\operatorname{Chr} 4: 107,548,653)$ \\
& & & & $4.3^{\#}($ Chr5: 53,611,846) \\
\hline
\end{tabular}

Notes: $\mathrm{LOD}=$ logarithm of the odds score based on traditional simple regression; $\mathrm{Chr}=$ chromosome; $\mathrm{CI}=$ confidence interval; $\#=$ meets suggestive genome-wide threshold; $\mathrm{F}=$ female; $\mathrm{M}=$ male. Bold text indicates overlapping QTL sharded by mobility and hypothermia (male) traits and italicized text indicates QTL detected only using GEMMA. 
detected only by the linear mixed-model mapping method for analgesia (Chr 5) response traits.

\section{Effectors of Endocannabinoid Signaling Identified as Positional Candidates for THC Initial Response QTLs}

As a first step towards identification of variants and genes modulating initial responses to THC, we prioritized several suggestive QTLs for exploration of putative candidate genes From Table 3-3, we selected the Chr 11 locus modulating both mobility and male hypothermia in response to THC and loci on Chrs 19 (male hypothermia), 13 (female hypothermia), Chr 1 (analgesia), and Chr 4 (analgesia) that were replicated using both HK and GEMMA mapping methods. Following a standard workflow we identified a number of genes near or overlapping SNPs, InDels, or SVs, some of which are predicted to have an impact on transcript or protein integrity (e.g. missense, stop gain or loss, splice region variant, frameshift). Based on evidence in support of genetic regulation of gene expression by cis eQTLs in naïve BXD brain tissue and literature associations with relevant cannabinoid terms, we nominated several high priority positional candidates for each prioritized QTL. Top positional candidates include $N d u f s 2$ (Chr 1 Analgesia QTL); Scp2 (Chr 4 Analgesia QTL); Rps6kb1/p70s6K (Chr 11 Mobility and male Hypothermia QTL); Pde4d (Chr 13 female Hypothermia QTL); and Pten (Chr 19 male Hypothermia QTL) (Table 3-4). These genes are likely to play a role in modulating initial response to THC based on location within modulatory QTL, genetic variation between the progenitor strains of the BXDs, evidence of genetic regulation of gene expression in BXD brain tissue, and previously reported involvement in endocannabinoid/cannabinoid receptor signaling pathways. Below we summarize the evidence in support of these top positional candidates.

NADH dehydrogenase [ubiquinone] iron-sulfur protein $2(N d u f s 2)$ is a positional candidate for modulation of the analgesic response to THC. Ndufs 2 encodes a core subunit of the mitochondrial Complex 1 . The gene also contains both missense and putative splice region variants and demonstrates evidence of genetic regulation of expression in the form of cis eQTLs in naïve BXD hippocampus, hypothalamus, neocortex, nucleus accumbens, and prefrontal cortex. Moreover, Ndufs 2 has been implicated in mediating response to cannabinoids. Activation of mitochondrial CB1Rs resulted in a decrease in PKA-dependent phosphorylation of oxidative phosphorylation proteins, including NDUFS2, and a concomitant decrease in brain mitochondrial function that was associated with cannabinoid-induced synaptic depression and amnesia [170].

Sterol carrier protein 2 (Scp2) is another positional candidate for modulation of the analgesic response to THC. The SCP2 protein has been proposed to regulate brain endocannabinoid levels and, thus, endocannabinoid system function [334, 335]. The Scp2 gene contains a putative splice region variant and shows evidence of genetic modulation of expression (cis eQTL) in naïve BXD neocortex and prefrontal cortex. 
Table 3-4. Summary of genes with high impact variants within QTL CIs.

\begin{tabular}{|c|c|c|c|c|c|c|}
\hline Position & Genes & $\begin{array}{c}\text { SNP } \\
\text { or } \\
\text { InDel }\end{array}$ & SV & $\begin{array}{c}\text { High } \\
\text { Impact }\end{array}$ & $\begin{array}{c}\text { cis } \\
\text { eQTL }\end{array}$ & Priority Genes \\
\hline $\begin{array}{l}\text { Chr 11: Mobility and } \\
\text { Hypothermia (M) }\end{array}$ & 212 & 130 & 34 & 60 & 33 & 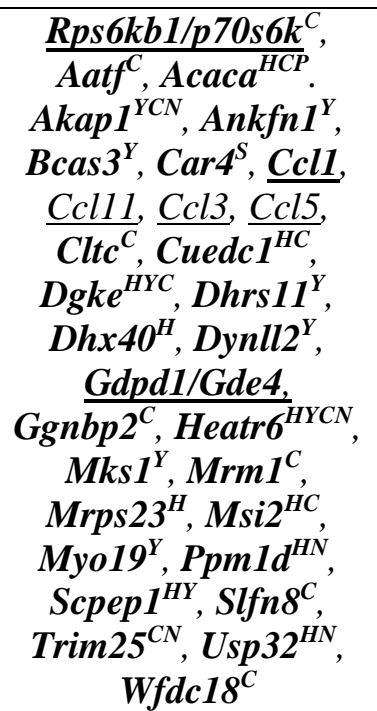 \\
\hline Chr 19: Hypothermia (M) & 105 & 53 & 6 & 3 & 5 & $\begin{array}{c}\underline{\text { Pten }}^{N}, \\
2700046 G 09 \text { Rik }^{\text {HYCN }} \\
\text { Atad1 }^{\text {HN }}, \text { Papss }^{\text {HCN }}\end{array}$ \\
\hline Chr 13: Hypothermia (F) & 107 & 46 & 8 & 8 & 12 & 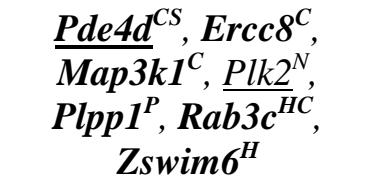 \\
\hline Chr 1: Analgesia & 91 & 60 & 11 & 29 & 23 & 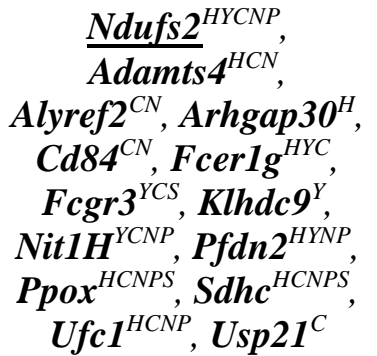 \\
\hline Chr 4: Analgesia & 107 & 68 & 15 & 14 & 16 & 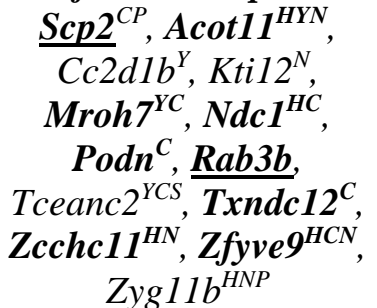 \\
\hline
\end{tabular}




\section{Table 3-4. (Continued).}

Notes: Number of genes or noncoding RNA (ncRNA) within each interval (Genes column), number of genes or ncRNA near or overlapping a SNP or InDel (SNP or InDel column), number of genes or ncRNA overlapping a SV ( $S V$ column), and number of genes or ncRNA containing a high impact SNP or InDel (High Impact column) are shown for each THC initial response QTL. Number of genes within each QTL CI whose expression is regulated by a cis eQTL are shown in the cis eQTL column. High priority candidate QTL genes (QTGs) associated through literature searches with

endocannabinoid/cannabinoid signaling whose expression (underline), regulated by a cis eQTL in at least one brain region ( $\mathrm{Y}=$ hypothalamus, $\mathrm{C}=$ neocortex, $\mathrm{S}=$ striatum, $\mathrm{N}=$ nucleus accumbens, $\mathrm{P}=$ prefrontal cortex) and/or overlap a SV or high impact SNP or InDel (bold) are shown in the Priority Genes column. 
Ribosomal Protein S6 kinase (Rps6kbl) is a positional candidate for modulation of the effects of THC on both mobility and hypothermia in males. The gene Rps $6 \mathrm{kbl}$ encodes the 70-kDa ribosome protein S6 kinase (p70S6K) and contains a predicted splice region variant. Expression of Rps6kbl is modulated by a cis eQTL in neocortex. Activation of the G-protein coupled CB1R by THC triggers cascades of intracellular signaling, including activation of phosphoinositide-3 kinase (PI3K)/Akt/glycogen synthase kinase 3 (GSK-3) and subsequent activation of the serine/threonine kinase mammalian target of rapamycin (mTOR). P70S6K is a downstream target of mTOR whose activation is associated with protein synthesis and regulation of different cellular states (e.g. survival, growth, or autophagy). The CB1R/mTOR/p70S6K signaling pathway in GABAergic interneurons in hippocampus was found to mediate THC-induced long-term memory deficits $[323,336]$

Phosphodiesterase 4D, cAMP specific $(P d e 4 d)$ is a positional candidate for modulation of the hypothermic response to THC in females. Phosphodiesterase 4 hydrolyzes cAMP and plays a role in mediating cAMP signaling pathways and neuroadaptations favoring drug reinforcement, tolerance and dependence [337-340]. Phosphodiesterase inhibitors have shown some efficacy in reducing drug seeking behavior or intake of psychostimulants, alcohol, and opioids in preclinical models [341]. There are four isoforms of Phosphodiesterase 4, including the positional candidate PDE4D, which expresses 11 alternative splice variants and exhibits widespread expression in brain [341]. Expression of Pde4d is modulated by cis eQTLs in naïve BXD neocortex and striatum and the gene locus contains a missense variant as well as a putative splice region variant.

Phosphate and tensin homolog (Pten) is a positional candidate for the modulation of the hypothermic response in males. Highly expressed in brain, Pten encodes a dual protein and lipid phosphatase enzyme that has been proposed to interact with neurotransmitter receptors, including NMDA receptors (Ning et al. 2004) and serotonin 5-HT2C receptors [342], and regulate their activity. Disruption of the interaction between PTEN and serotonin 5-HT2C receptors in the ventral tegmental area (VTA) has been shown to inhibit the firing rate of dopaminergic VTA neurons projecting to the nucleus accumbens [342]. Moreover, disruption of this interaction also blocked the ability of THC to enhance the firing rate of dopaminergic VTA neurons and subsequent THCinduced conditioned place preference [342] suggesting that interactions between PTEN and serotonin receptors may mediate some of the behavioral effects of cannabinoids and other drugs of abuse [343]. The expression of Pten in naïve BXD nucleus accumbens is modulated by a cis eQTL and there are 59 variants located within or near the gene locus, although the impact of these variants on gene function or expression is not clear.

\section{Positional Candidates Associated with Other Drugs of Abuse}

For each high priority positional candidate or QTL gene (QTG) we explored broader associations with over 300 addiction and psychiatric disease key words using a newly developed resource: Relationship with Addiction Through Searches of PubMed 
(RatsPub). This search finds sentences in PubMed that contain both the gene of interest and keywords relevant to drugs of abuse, addiction, and psychiatric disease. Our overall goal was to place provisional endocannabinoid signaling QTGs identified in our study into a broader biological context. Given the broad modulatory effects of the endocannabinoid system on brain function, we hypothesized that THC initial response QTGs would also be associated with diverse signaling in response to other drugs of abuse and psychiatric disorders.

Pten (Chr 19 male Hypothermia QTG), Rps6kb1/p70S6K (Chr 11 Mobility and male Hypothermia QTG), and Pde4d (Chr 13 female Hypothermia QTG) were the most well connected QTGs in terms of associations with drugs of abuse and psychiatric disease. All three of these QTGs are associated with terms related to stress, anxiety, depression, and schizophrenia. Rps6kb1/p70S6K and Pten were associated with terms related to alcohol, amphetamine, cocaine, nicotine, opioid, phychedelics and sensitization. Pde $4 d$ was associated with terms related to nicotine and psychedelics. Pten, $R p s 6 \mathrm{~kb} 1 / \mathrm{p} 70 \mathrm{~S} 6 \mathrm{~K}$, and $P d e 4 d$ were also associated with neuroplasticity, neurotransmission, signaling and transcription terms in support of their known roles in these biological processes. Moreover, these three QTGs have also been implicated in brain regions relevant to drug abuse and psychiatric disease as evidenced by their literature co-citation with relevant terms (e.g. accumbens, amygdala, cortex, habenula, hippocampus, or striatum).

Although less well-connected, the QTGs $N d u f s 2$ (Chr 1 Analgesia QTL) and Scp2 (Chr 4 Analgesia QTL) were also associated with neuroplasticity, signaling, transcription, stress, and hippocampus terms. In addition, $N d u f s 2$ was associated with schizophrenia terms and $S c p 2$ was associated with alcohol-related terms.

\section{Initial Response of THC and Other Drugs Correlate}

Recent studies quantified initial response to several drugs of abuse (i.e. cocaine, alcohol, morphine) or intravenous cocaine self-administration among a large set of BXD RI strains [265,266]. BXD traits associated with these studies are available in GN and are of sufficient strain depth to facilitate a moderately well-powered and exploratory correlation analysis with THC initial response traits. We hypothesized that strong correlations between initial response to THC and initial response or self-administration of other drugs of abuse among BXD strains was indicative of shared genetic and/or biological co-regulation.

From our background list of 314 BXD legacy traits, we found 45 significant $(p<$ $0.05)$ correlations between 43 drug response or self-administration traits and one or more of our THC initial response traits. Several interesting correlation patterns were evident between initial responses to THC and BXD legacy cocaine, ethanol, and morphine response traits (Figure 3-5). One of the most striking patterns was an enrichment of significant and positive correlations between THC-induced analgesia and initial locomotor responses to morphine $(50 \mathrm{mg} / \mathrm{kg}$ i.p.) from 90 to $150 \mathrm{~min}$ post-injection. 


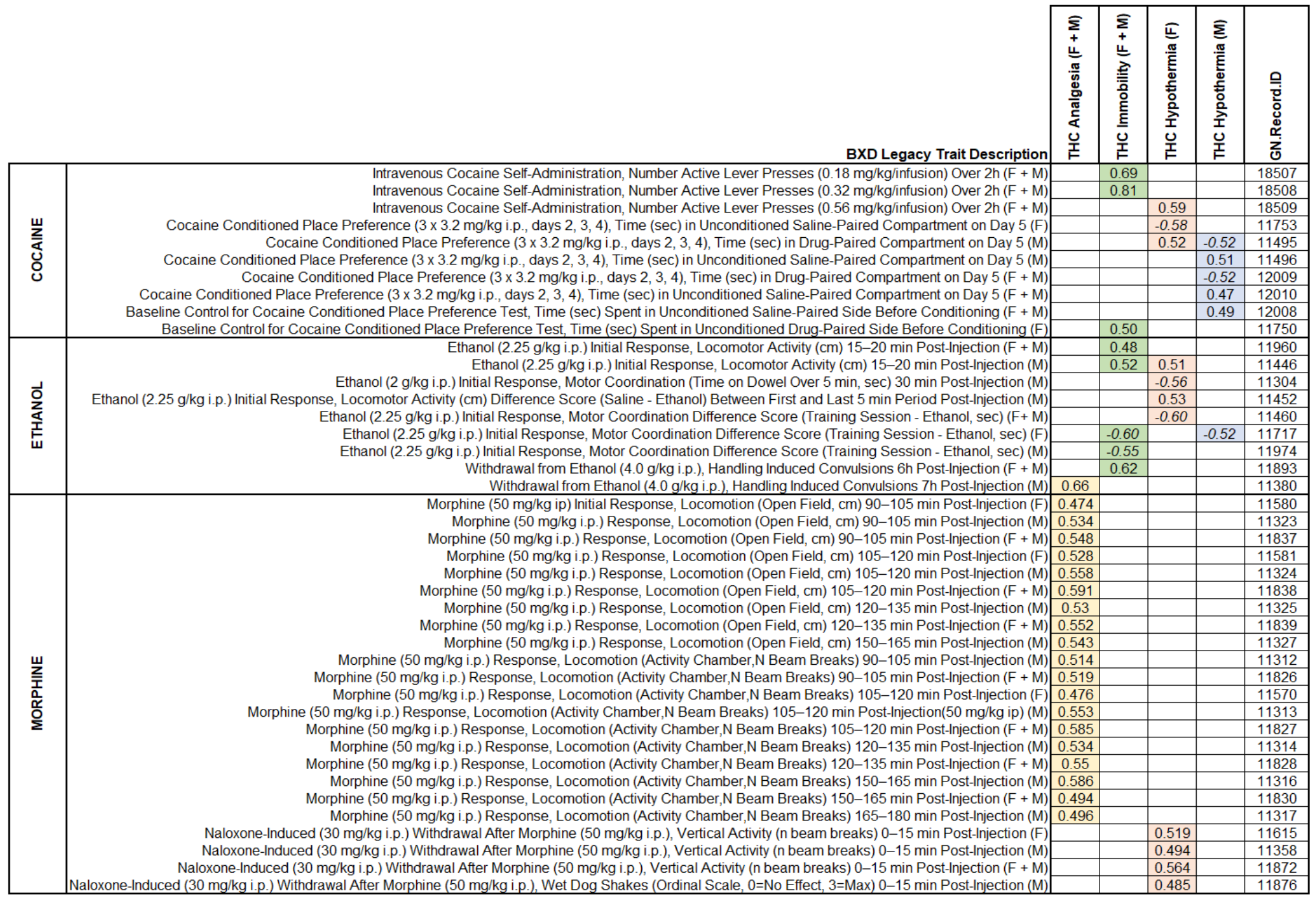

Figure 3-5. Summary of significant correlations between initial response to THC and BXD legacy drug response traits. 
Other patterns emerged as well: (1) significant and positive correlations between THC-induced hypothermia (females) and naloxone-induced (30 mg/kg i.p.) withdrawal from morphine (50 mg/kg i.p.), (2) significant and positive correlations between cocaine self-administration and THC-induced immobility, (3) sex-specific and significant correlations between THC-induced hypothermia and preference for cocaine, and (4) significant correlations between both THC-induced immobility and hypothermia (females) and initial motor response and tolerance to ethanol.

The role of sex on trait covariation was not explicitly quantified in this exploratory analysis, however, strong correlations were detected between each THC trait and both sex combined $(\mathrm{F}+\mathrm{M})$ and sex separated $(\mathrm{F}$ or $\mathrm{M}) \mathrm{BXD}$ legacy trait measurements. Finally, correlation patterns did not appear to be driven by genetic coregulation of cannabinoid and drug response traits from shared loci (i.e. peak QTLs for correlated traits were non-overlapping). Instead these correlation patterns indicate shared biological mechanisms and/or more complex genetic modulation driving response to THC and other drugs of abuse.

\section{Discussion}

In this study we show that strain differences in initial responses to THC between B6, D2, and their BXD progeny are heritable (Figure 3-2 and Table 3-2). Thus, genetic mapping of THC response traits in the BXD family will lead to the identification of genomic loci, variants, and genes that modulate trait variation. Candidate QTGs will almost certainly be direct or indirect effectors of endocannabinoid signaling given that the THC response traits measured in our study are entirely downstream of CB1R signaling. To this end, we identified multiple QTLs and positional candidate endocannabinoid signaling QTGs (Pten, Rps6kb1/p70S6K, Pde4d, Ndufs2, Scp2) that may control variation in initial response to THC (Tables 3-3 and 3-4). Many of these candidates mediate responses to other drugs of abuse. Moreover, we found significant correlations between initial responses to THC and behavioral responses to cocaine, alcohol, and morphine (Figure 3-5). Taken together, we provide strong evidence that gene variants in endocannabinoid signaling pathway genes are responsible for individual variation in initial THC response and are likely to cause variation in the behavioral responses to other drugs of abuse.

Our genetic screen for natural variation in traits modulated by effectors of endocannabinoid signaling was designed to identify specific genes and pathways involved in the response to THC. Targeted genetic deletion has revealed insight into the integral role of CB1R in response to THC. However, little is known about the role of natural variation in response to cannabinoids. A handful of genes (e.g. CADM2, NCAM1, $N R G 1, C S M D 1$, and CHRNA2) have been associated with cannabis use or dependence in human genome-wide association studies [244-247, 344]. The underlying mechanisms whereby these genes mediate use or dependence to cannabinoids or interact with the endogenous cannabinoid system has yet to be elucidated, with one notable exception. Heterozygous Nrgl (Neuregulin) mutant mice, in which one copy of the gene contains a 
deletion of the transmembrane region, are more sensitive to the initial locomotor suppressant and behavioral effects of THC [345] and develop more rapid tolerance to the motor suppressant effects [346] relative to control mice. Differences in cannabinoid responses were accompanied by modest increases of CB1R levels in substantia nigra and significant decreases in both thalamic NMDA receptor levels and striatal dopamine D2 receptor levels in naïve heterozygous $\mathrm{Nrgl}$ mutant mice relative to controls [347] . Association of $N R G 1$ variants with cannabis dependence in humans and independent preclinical evidence for a role of $\mathrm{Nrgl}$ in initial response to cannabinoids is striking and suggests that identification of additional genes that modulate endocannabinoid signaling and cannabinoid response is warranted.

To date, no human studies have addressed the impact of genetic variation on acute or chronic pharmacological or behavioral responses to cannabis or derived cannabinoids. Greater understanding of the genes and pathways mediating responses to cannabinoids is important for at least two reasons. First, identification of variants in endocannabinoid signaling pathways will help us better understand behavioral responses to cannabinoids, including risk of cannabinoid dependence, and provides the opportunity to decouple unwanted side effects with therapeutic properties of cannabinoid drugs (e.g. motor impairment versus analgesic effects of THC). Second, endocannabinoid pathways represent common points of convergence highly relevant for understanding the molecular response to many drugs of abuse and the biological mechanisms underlying addiction processes.

There are strong correlations between initial response to THC and initial response or self-administration of cocaine, morphine, and alcohol among BXD strains that could be indicative of shared genetic and/or biological co-regulation. The endocannabinoid system (e.g. lipid ligands, biosynthetic and catabolic enzymes, G-protein coupled receptors and effectors of signaling) plays an important role in modulating synaptic plasticity, behavior, and reward circuitry. Cannabis, cannabinoids, and non-cannabinoid drugs of abuse have been shown to disrupt endocannabinoid system function and signaling, and these changes may contribute to addiction processes [348]. For example, exposure to alcohol and opioids has been shown to increase the level of endocannabinoid system ligands (e.g. AEA or 2-AG) in rodent brain reward regions [349-352]. Moreover, activation of CB1Rs in some rodent models has been shown to enhance the rewarding effects of alcohol and opioids through both dopamine dependent and independent pathways [353-355]. Both cocaine [356-359] and alcohol [360,361] exposure has been shown to alter CB1R-mediated plasticity in rodent brain reward regions (e.g. striatum, VTA, NAc, and/or BNST).CB1R-mediated plasticity and signaling is also important for mediating response to opioids $[362,363]$. Thus, the endocannabinoid system converges at many points with other neurotransmitter systems to mediate drug response and alter behavior following drug exposure.

We hypothesized that correlations between initial response to THC and responses to alcohol, cocaine, or opioids in the BXD population resulted from the convergence of genes and pathways that mediate both effects. Indeed, we observed striking correlations between THC and opioid responses (enhanced sensitivity to the analgesic effects of THC 
and enhanced sensitivity to the motor stimulant effects of morphine), THC and cocaine responses (enhanced sensitivity to the motor suppressant effects of THC and higher active lever presses for cocaine), THC and ethanol responses (enhanced sensitivity to the motor suppressant and hypothermic effects of THC and enhanced sensitivity to the motor stimulant effects of ethanol), and THC and withdrawal from alcohol and opioids (enhanced sensitivity to the motor suppressant effects of THC and higher levels of withdrawal from alcohol in contrast to lower levels of precipitated withdrawal from opioids). The results of this exploratory correlation analysis suggest underlying shared genetic and biological regulation. Of interest, no single shared locus could account for the covariance in drug response traits, indicating complex regulation by multiple loci, genes, and variants. Importantly, our exploratory analysis identified individuals of the BXD population with extreme responses to multiple drugs of abuse. These individuals can be leveraged to directly test predictions about drug response and to identify mediators of behavioral responses to both cannabinoid and non-cannabinoid drugs using both genetic (QTL mapping) and pharmacological (through targeting of specific pathways) methods.

Our study marks the first and largest attempt to quantify genetic factors mediating initial responses to THC in males and females of a rodent genetic population. While we have made an important contribution to the field, there are some limitations of our study that will need to be addressed by future experiments. First, THC traits were profiled in a subset of 20 BXD strains which limits our ability to detect QTLs of small effect size. All QTLs identified in our study are suggestive after accounting for genome-wide testing. It is likely that these loci will be replicated in an independent or larger cohort of BXD strains. We emphasize that this replication will be required to confirm QTLs and QTGs prioritized by our study. Other aspects of our study design limit translatability somewhat and should be addressed by future studies. These include the use of a single major component of cannabis (THC), use of a single dose $(10 \mathrm{mg} / \mathrm{kg})$, and quantification of acute physiological responses to THC as opposed to chronic exposure, dependence and/or withdrawal. Finally, we were agnostic to genetic variation in cannabinoid metabolic pathways in this study.

QTL mapping of all traits resulted in a few interesting peaks, mostly at the suggestive level, with peak values increasing when mapping with GEMMA. The lack of strong QTLs is not surprising when you take into account the preliminary number of BXD strains used in this study $(\mathrm{N}=20)$. The lack of power 20 strains provides can be demonstrated by a more established dataset. The experiment involving morphine hypolocomotion contains 64 BXDs and has a strong, pronounced QTL peak on chromosome 10 (5 Mb, 39.33 LRS, Figure 3-6). When strains are randomly removed down to 40 strains it still holds significant, but has a large drop in value (LRS 20.9). When cut down to 20 strains, the number used in this study, the peak drops below significance and is now at a similar level of suggestive that the THC trait peaks are located at (12.85 LRS). Using a power analysis tool developed by David Ashbrook at UTHSC and colleagues, based on traits average heritability and locus effect sizes we would need to increase strain number somewhere between 40 and 50 strains to obtain significant QTL peaks (Figure 3-7). This simulation might also provide some support that a trait with a normal distribution in the BXD population and a suggestive QTL when 

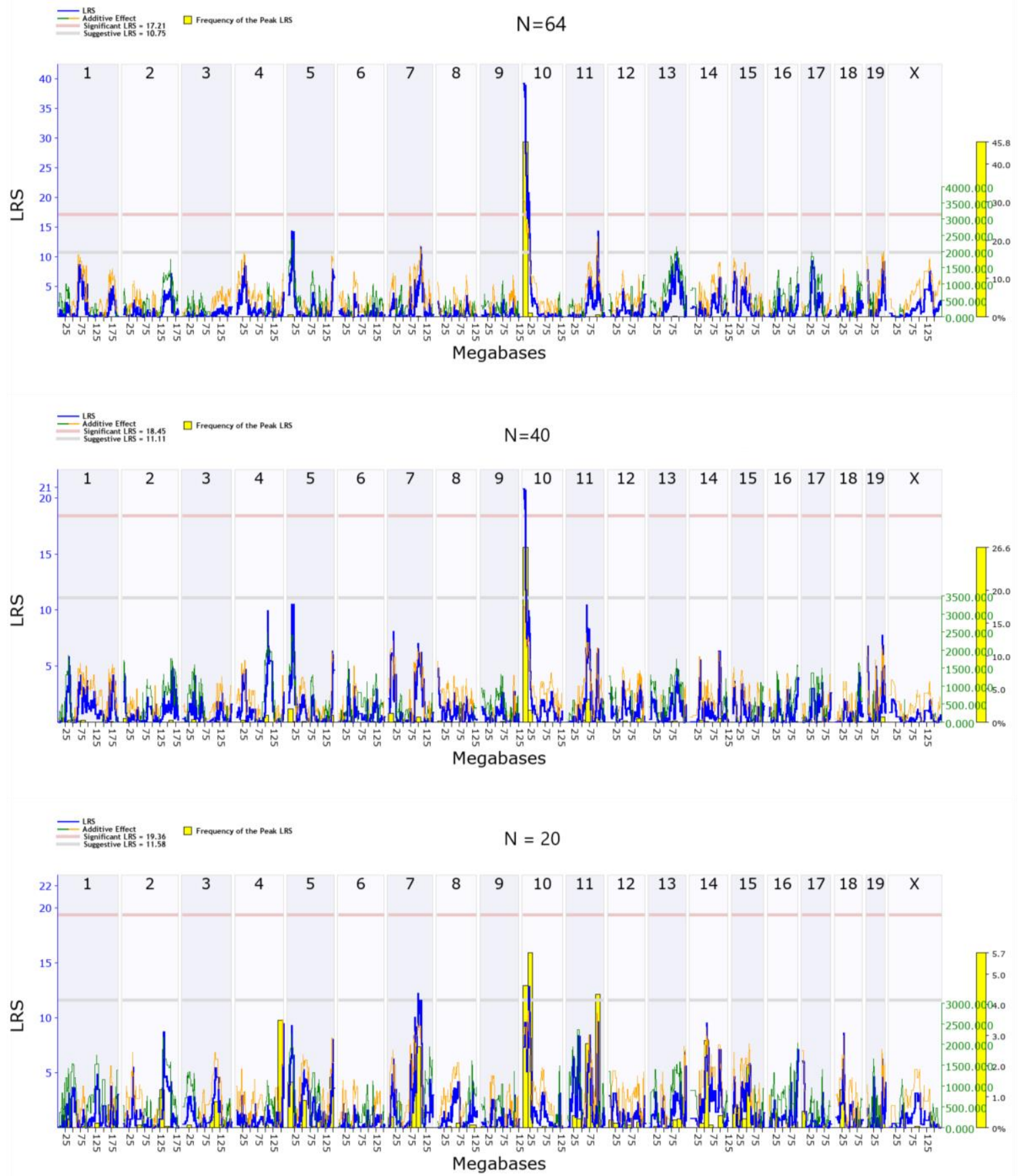

Figure 3-6. Exercise in mapping power with number of BXD strains.

Using existing morphine hypolocomotion data in GeneNetwork to demonstrate the loss in power when randomly reducing the number of BXD strains from its original 64 down to 20. GeneNetwork trait ID for data is 11312 . 


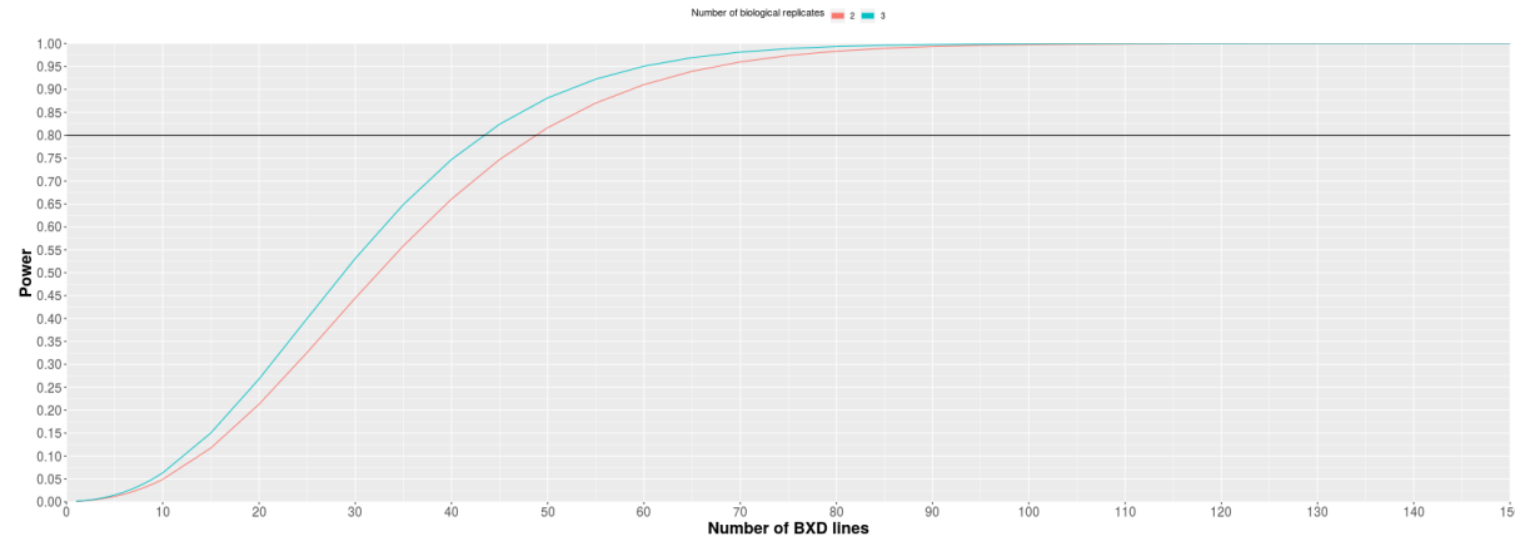

Figure 3-7. Power calculation of BXD family

Power calculator that estimates how many BXD strains needed before having significant QTLs when mapping. A heritability of 0.5 and locus effect size of 0.4 was used based on preliminary data with replicates of 2 or 3 mice per group. For QTL peaks to reach significant levels we would need to increase strain number between 40 and 50 . 
mapped with a small number of strains might reach later reach significance at the same QTL when a larger number of strains are tested.

Drug metabolism is an important biological process that impacts response to drugs of abuse and susceptibility to addiction. Based on trait correlation structure and genetic mapping, it is unlikely that strain differences in THC metabolism contributed to trait variation in all three of our initial response traits (e.g. mobility, hypothermia, and analgesia). It will be important in the future to establish whether sex or strain differences in THC metabolism contribute to variation in response and whether there are functional variants that impact THC metabolism in rodents. Several functional human variants (alleles) have been identified for cannabinoid metabolism genes [364]. The impact of these variants on response to cannabinoids and addiction is not clear, although a small study reported a trend towards increased sensitivity to THC in individuals homozygous for the CYP2C9*3 allele associated with slower clearance of THC [365].

Ultimately, our study demonstrates the feasibility of genetic mapping in the BXDs to identify underlying biological and genetic factors contributing to response to THC and other drugs of abuse. We have taken important first steps towards the identification of loci and genes that modulate initial responses to THC. We have also provided evidence of covariation among drug response traits, although the underlying causal factors remain elusive. Finally, we have identified BXD individuals with extreme responses to acute THC exposure. Identification of BXD individuals with greater initial sensitivity to THC will facilitate more detailed genetic analysis of intake, reward, withdrawal, tolerance, and the behavioral impact of cannabinoids on motivation, cognition, and health. The genetic architecture of behavioral and pharmacological response to cannabis and cannabinoids is difficult to reconstruct using currently available human data sets. A better understanding of trait correlation structure and regulation by underlying genetic variation in diverse rodent models is expected to help bridge these gaps and direct the focus of future human studies. Development for people suffering from cannabis use disorder and could also lead to screening for at-risk individuals who would be more susceptible to marijuana's adverse health effects. 


\section{CHAPTER 4. CONCLUSIONS AND FUTURE DIRECTIONS}

\section{Conclusions}

There are significant strain and sex differences in the cannabinoid-induced tetrad test between two genetically diverse inbred mouse strains, B6 and D2. We also demonstrate significant strain differences in the locomotor (immobility) and antinociceptive effects of THC. However, the hypothermic response to THC is more prominent in females. There are also prominent strain differences in the rapid tolerance of antinociceptive effects of THC and sex differences in hypothermic responses.

This is the first quantitative and comprehensive analysis of sex-by-strain differences in initial responses and tolerance to THC. The response traits measured here have been shown by other investigators [252-261] to be consequences of CB1R agonist activity. We have established that the differential responses in locomotor and analgesia traits are driven by genetic variants segregating between the two strains, as well as downstream effects mediate by CB1R signaling. Strain-by-sex differences cause variation in THC hypothermic responses.

The results from B6 and D2 strains warranted deeper investigation into variable THC response. The BXD recombinant inbred genetic reference population, derived from the B6 and D2 strains, provided a great source of genetic variation for testing. Testing these strains for THC response had many advantages including: (1) validating strain and/or sex differences, (2) determining heritability of the traits, (3) discovering correlations among the THC traits and with other drug response traits, and (4) data generation to begin exploring genetic mechanisms influencing these traits. All three tested traits are heritable and have significant strain differences, including hypothermia, which was not significant between the B6 and D2 parental strains. The sex difference in hypothermia in the parental strains held true among the BXD strains. THC response traits possess strong positive correlations with response traits from other drugs of abuse including alcohol, morphine, and cocaine, giving evidence for shared biological mechanisms underlying a variety of drug responses. Finally, we were able to begin the search for casual genetic mechanisms with QTL mapping and identifying candidate genes. Each trait had suggestive peaks, but one gene of interest, Rps6kb1, stood apart as an especially interesting candidate. The expression of this gene was regulated by a ciseQTL and there was published evidence linking Rps6kb1 to endocannabinoid pathway translational control and a role in modulating THC's amnesic properties. A potential limitation of the study was that Rps6kb1 possesses many sequence variants between B6 and D2 that segregate among the BXD strains. Thus, immediate identification of a causal variant with an impact on function is not possible without further experimental manipulation.

Strong statistical significance of genetic factors on THC response traits in parental strains combined with high trait heritability of traits in the data obtained from the BXD strains $(\mathrm{N}=20)$ gives great confidence for the involvement of genetics factors in THC 
responses. However, a lack of power became apparent with QTL mapping and the search for potential casual genes. Increasing the number of BXD strains would be expected to improve both the number of QTLs detected and peak significance levels leading to the discovery of more candidate genes for all traits. Other experiments need to be performed to corroborate the data and explore different physiological functions that could be affecting THC response traits. This may include validation of differential gene expression using qPCR and validation of sequence variants by traditional Sanger Sequencing methods.

\section{Phenotype Testing with Genetically Altered Mice}

One way to test if a gene is contributing to an observed phenotype is by manipulating the function of that gene. Genetic knockout (KO) models are a prominent experimentation tool for gene function in animal studies. Interestingly, a $\mathrm{KO}$ mouse model already exist for one of our candidate genes (Rps6kbl) and is available from Jackson Laboratory (Stock \#031508). Running these mice through the THC response pipeline would be an interesting start, but the background strain for this mouse is $\mathrm{BALB} / \mathrm{cByJ}$. Results from a KO model with the higher expressing B6 strain would provide better comparison and stronger evidence with the current data to the effect of eliminating this gene. To accomplish this a new KO mouse with $\mathrm{B} 6$ background needs to be created or Rps6kbl expression manipulated through other technologies.

Modulation of gene expression has been traditionally accomplished through transduction (viral) or transfection (nonviral). These are methods that insert foreign DNA or RNA into a cell, producing genetically modified cells or organisms. These techniques, particularly viral mediated methods, have become more common in studies involving behavior and the CNS [326]. Some attractive attributes of using viral transduction in vivo is the ability to inject the virus in a certain region and design the virus to infect specific cell types. These methods could be used to knockdown gene expression of Rps6kb1 in B6 mice in the striatum to see its effects on locomotion and temperature in response to THC.

A second method that could be used is the more recent, but well established CRISPR/Cas 9 technique [327]. With this technology specific loci in the genome can be targeted for gene editing. In summary, a guide RNA (gRNA) is attached to the Cas 9 nuclease and directs the nuclease to the target of interest where the DNA is cleaved. At this site you can perform precise gene editing and introduce purposeful mutation, such as reversing a SNP, or allow the cell to undergo the error prone non homologous end joining DNA repair pathway, introducing mutations and effectively knocking out the target gene. In a previous study our lab performed the former. A non-coding variant in GABRA2 that reduced protein expression levels was identified in the B6 strain [328]. Using the CRISPR/Cas 9 system with a DNA repair template this variant was reversed and GABRA2 protein levels were restored. If the causal variant were to be identified in $R p s 6 \mathrm{~kb} 1$ this same approach can be used in the D2 strain to see if they become more sensitive to the hypolocomotion effects of THC. CRISPR/Cas 9 can also be used on Rps6kb1 in the B6 strain to $\mathrm{KO}$ the gene, potentially making it more resistant to the hypolocomotion effects. 


\section{Investigation into the Pharmacogenetics of THC Response}

\section{Distribution and Metabolism of THC}

Pharmacokinetics of Cannabis/THC exposure needs to be examined, specifically distribution and metabolism. Variable response of the drug might be influenced by these molecular processes. THC is a viscous substance that has high lipid solubility and low aqueous solubility. High THC concentrations are quickly reached in blood after use or exposure. The majority (90\%) of THC circulates through the body in plasma and the rest $(10 \%)$ is carried by red blood cells [329]. Once circulating, the highly lipophilic substance gets rapidly distributed in adipose and highly vascularized tissues, including the brain. THC is broken down by liver microsomes through hydroxylation and oxidation with enzymes of the cytochrome P450 (CYP) complex [329]. Hydroxylation of THC generates the primary active metabolite 11 -hydroxy- $\Delta 9$-tetrahydrocannabinoal $(11-\mathrm{OH}-$ THC) and further oxidation produces the primary inactive metabolite 11-nor-9-carboxy$\Delta$ 9-tetrahydrocannabinol (THC-COOH). Route of administration (inhalation vs ingestion) significantly impacts the bioavailability of THC. Extensive liver metabolism that goes with ingestion results in much higher 11-OH-THC concentration and less unmetabolized THC in plasma and tissue compared to inhalation, which bypasses first past metabolism [330]. While break down of this drug occurs relatively rapidly with either route of administration, it is important to note that residual levels of THC can be maintained in the body for many days following exposure.

Despite the straightforward outline mentioned above, it is possible that genetic variation could be altering these processes, contributing to differential response to the drug. As with the phenotypic data described in previous chapter, the BXDs along with their parental strains could be leveraged to identify genetic factors contributing to variation in THC metabolism. The parental strains will be sampled to establish heritable differences between these two diverse inbred strains. A search for candidate genes using the BXD progeny can then be performed if significant differences are found between B6 and D2. To mirror the initial response phenotypes studied above, blood and brain tissue will be collected after a single injection of THC $(10 \mathrm{mg} / \mathrm{kg})$ at varying time points $(5,10$, 15, 30, 60, and 120 minutes). THC and relevant metabolite concentrations in blood and brain tissue can establish differences in metabolism, absorption, clearance, and disruption. It could be fruitful to include a chronic paradigm of daily injections for approximately two weeks. Results from chronic use could give insight into the relevance of pharmacogenetics with abuse and dependence.

Based on the significant phenotypic differences seen in the parental strains and the $20 \mathrm{BXD}$ lines used, gene variants relating to metabolism, absorbance, or clearance of THC are potentially playing a role in the wide range of responses. If higher concentrations of THC are being absorbed into certain brain regions, we would expect it to correlate with known behavioral phenotypic behavior (ex. high striatum THC levels in strains with high levels of hypolocomotion). After using at minimum the same number of BXD strains as before, but preferably more, we would expect to have QTL mapping 
results and the ability to generate a list of candidate genes involved with the metabolism, clearance, and distribution of THC following exposure.

\section{CB1R Receptor Dynamics and Relevant Signaling Pathways}

Another area of interest is testing if genetic variation is affecting the biochemical and molecular pharmacodynamics of the endocannabinoid system, particularly the CB1 receptor and other pertinent pathways affecting individual response to THC. Based on behavioral data (Chapters 2 and 3) and what is known about drug response in certain brain regions, the striatum, hippocampus, and cortex would be reasonable candidate brain regions of interest. Brain tissue collected for the metabolic study will be used to look at receptor dynamics. RNA sequencing and proteomics will be used in relevant brain tissues at the established time points to investigate gene expression and protein level changes after exposure. To visualize the internalization and desensitization process after receptor stimulation, immunohistochemistry probing techniques can be used on brain slices.

We have generated preliminary data in this area of investigation performing shotgun proteomics from striatal synaptosomal preps on a small number of unexposed male B6 and D2 parental strains [281]. Aside from molecular activity that occurs upon $\mathrm{CB} 1$ activation, we wanted to look at basal levels of CB1R to ensure any observed differences were due to pharmacodynamics rather than receptor expression levels. A significant strain difference in baseline CB1R expression level was identified (Figure 4-1A). These results were confirmed with western blot, giving near identical results (Figure 4-1B). A baseline difference was observed between B6 and D2 male mice. This baseline difference came as an interesting surprise since there are no strong variants in CB1R gene, CNR1, that are predicted to impact expression levels. One potential cause of this strain variation in receptor level is post-translational regulation such as modification (i.e. phosphorylation), proteolysis, or sequestration. Another potential cause is a downstream alteration such as endocannabinoid levels, G-protein or $\beta$-arrestin signaling differences, or protein expression of a receptor CB1R interacts with. Expression levels of CB1R could also be mediated by other genes. This needs to be further investigated by including females, BXD strains, and looking at other regions of the brain.

GeneNetwork contains RNA sequencing and microarray datasets from a multitude of brain regions. Analysis of genes involved in the cannabinoid signaling pathway within the BXD and parental strains was analyzed from these datasets to generate preliminary data. Many genes had strain and strain-by-region expression significance indicating that genetic factors may cause differences in cannabinoid signaling gene expression leading to variation in phenotypic 


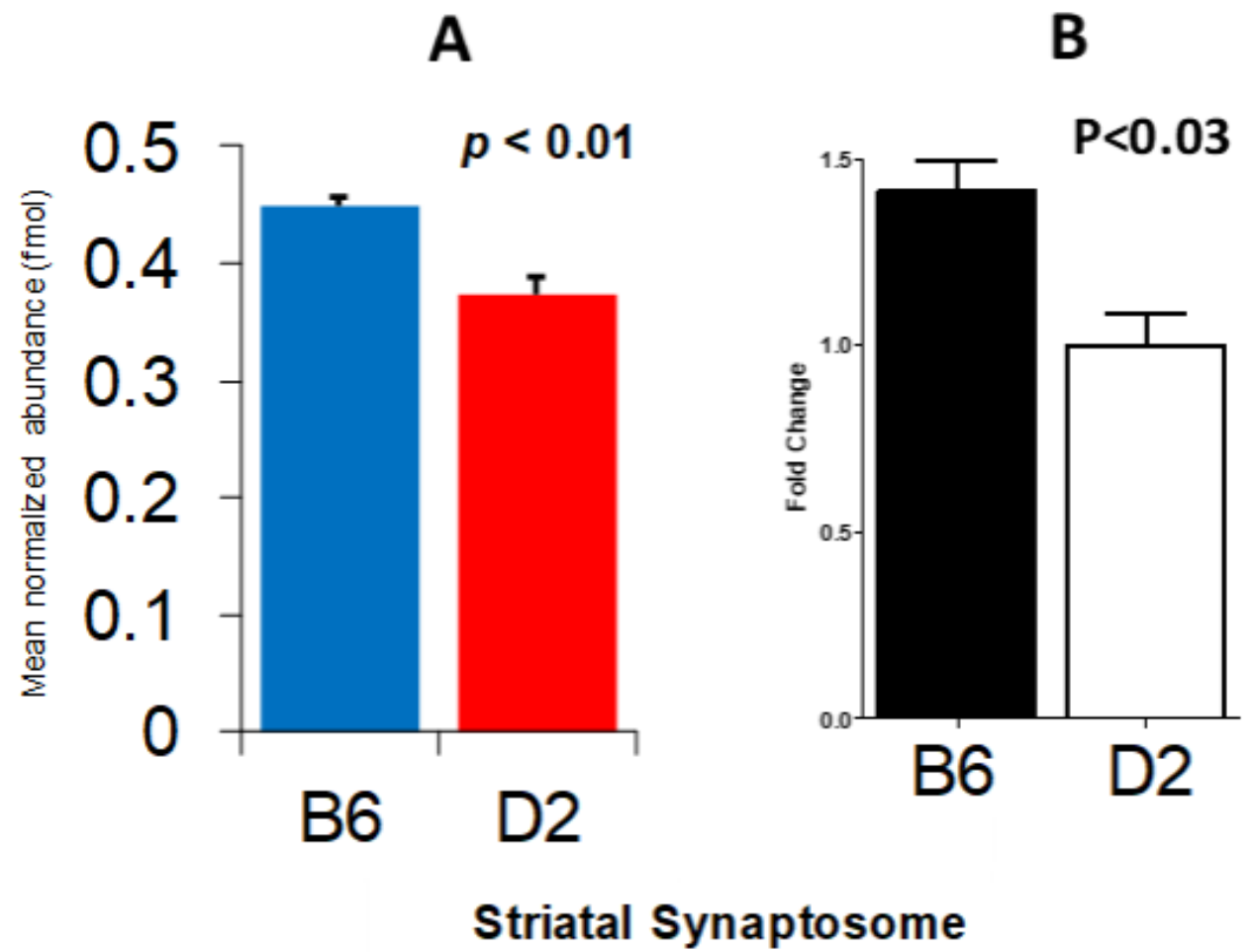

Figure 4-1. Baseline CB1 receptor differences between B6 and D2.

(A) Proteomics data generated using an LC-MS/MS revealing baseline differences in unexposed animals between male B6 $(\mathrm{N}=4)$ and $\mathrm{D} 2(\mathrm{~N}=4)$ strains in striatal synaptosomal preps. (B) Western blot quantification results confirming proteomics results. 
response. From this list of genes three stood out: Beta arrestin 2 (Arrb2), G-protein coupled receptor kinase 2 (Adrbk2), and G-protein sub-unit beta 2(Gnb1) (Figure 4-2).

These genes are controlled by cis-eQTLs indicating that a sequence variant between B6 and D2 located near the gene locus is controlling expression of the gene. Most importantly, all these genes have notable roles in CB1R signaling. Gnbl is an important subunit for G-protein heterotrimer formation, Adrbk2 modulates the phosphorylation and deactivation of CB1R, and Arrb2 targets CB1R for internalization. Phosphorylation, deactivation, and internalization are all important processes in the desensitization process upon receptor activation. These gene expression differences are among naïve mice. We would develop expression datasets from mice treated with THC with the same time points outlined above to investigate any expression differences with response to the drug.

After performing these experiments, a list of candidate genes along with their relevant brain regions can be generated. A list of other signaling pathways or modulators affecting or being affected by cannabinoid signaling could also emerge. An expanded knowledge of CB1R activation and desensitization, including other signaling pathways that could be affecting these processes, is needed to further the understanding of cannabinoid response and therapeutics. Expanding on this topic is far reaching. It could help with drug development for people suffering from cannabis use disorder, help identify individuals who are susceptible to addiction or the adverse health effects of cannabis use, or help prevent unwanted side effects when designing new drugs. Even without a full understanding of the CB1R signaling pathway, it is a potential drug target in many areas of disease including cancer [331], diabetes [332], and cardiovascular disease [333], eluding to how impactful closing these gaps in knowledge could be. 

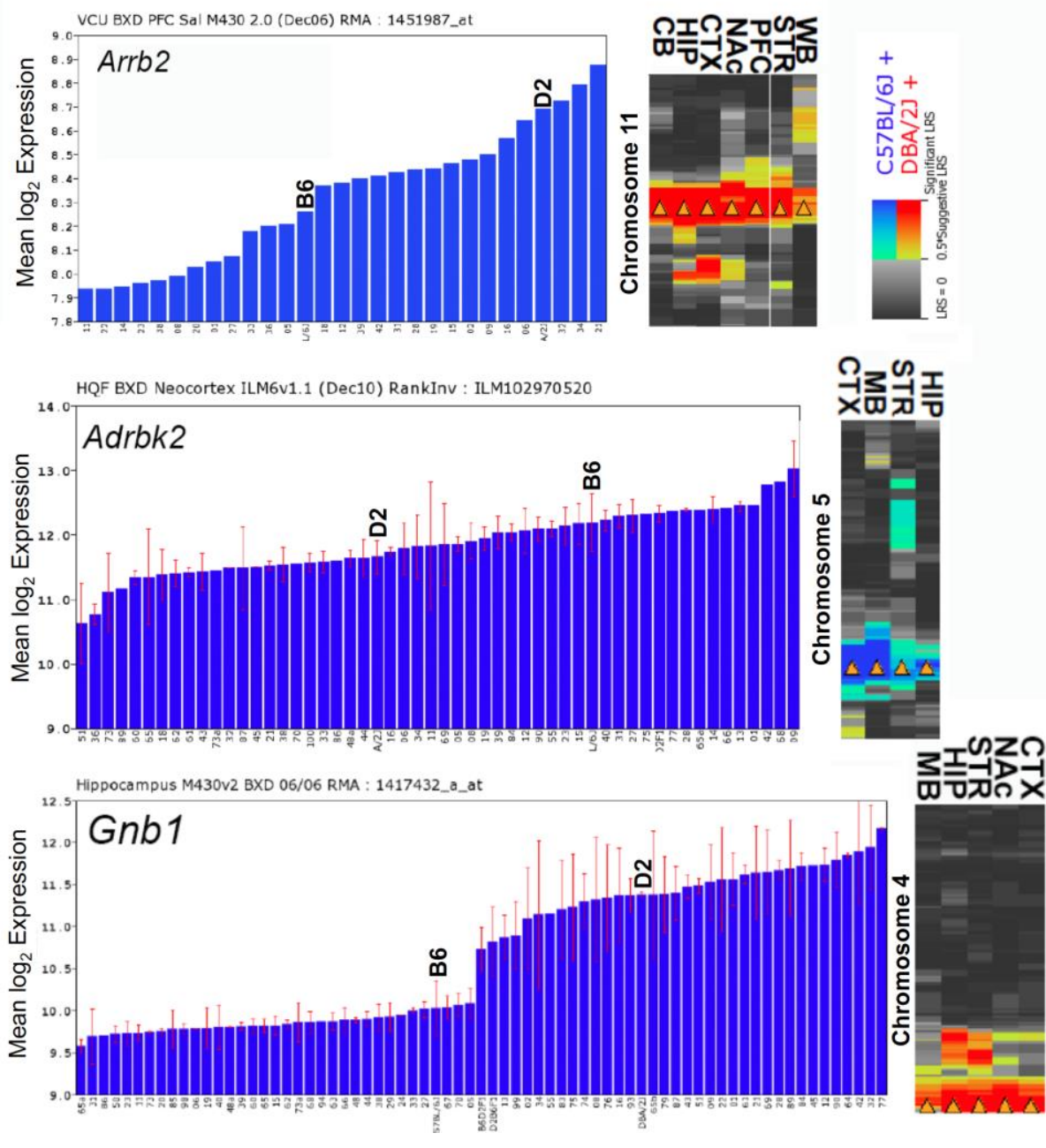

Figure 4-2. Gene expression differences in cannabinoid signaling genes across the BXD population.

Arrb2, Gnb1, and Adrbk2 expression is variable among the BXD population (blue bar charts). Expression of each gene is modulated by a cis-eQTL, (yellow arrowheads on heatmap). Rows indicate position on the chromosome and columns indicate expression in various brain regions. Color intensity indicates strength of association (LRS or LOD score) between cognate gene expression and genomic location. Red and blue indicate that D2 or B6 parental alleles drive higher expression of the gene, respectively. $\mathrm{Mb}=$ midbrain, Hip=hippocampus, $\mathrm{CTX}=$ cortex, NAc=nucleus accumbens. 


\section{LIST OF REFERENCES}

1. Mechoulam, R., The Pharmacohistory of Cannabis sativa, in Cannabis as Therapeutic Agent. 1986, Boca Raton, FL, USA: CRC Press:.

2. Pertwee, R.G., Cannabinoid pharmacology: the first 66 years. Br J Pharmacol, 2006. 147 Suppl 1: p. S163-71.

3. Gaoni, Y. and R. Mechoulam, Isolation, structure, and partial synthesis of an active consituent of hashish. Journal of American Chemical Society, 1964. 86: p. 1646-1647.

4. Hanus, L.O., et al., Phytocannabinoids: a unified critical inventory. Nat Prod Rep, 2016. 33(12): p. 1357-1392.

5. Howlett, A.C., Inhibition of neuroblastoma adenylate cyclase by cannabinoid and nantradol compounds. Life Sci, 1984. 35(17): p. 1803-10.

6. Devane, W.A., et al., Determination and characterization of a cannabinoid receptor in rat brain. Mol Pharmacol, 1988. 34(5): p. 605-13.

7. Matsuda, L.A., et al., Structure of a cannabinoid receptor and functional expression of the cloned cDNA. Nature, 1990. 346(6284): p. 561-4.

8. Munro, S., K.L. Thomas, and M. Abu-Shaar, Molecular characterization of a peripheral receptor for cannabinoids. Nature, 1993. 365(6441): p. 61-5.

9. Devane, W.A., et al., Isolation and structure of a brain constituent that binds to the cannabinoid receptor. Science, 1992. 258(5090): p. 1946-9.

10. Sugiura, T., et al., 2-Arachidonoylglycerol: a possible endogenous cannabinoid receptor ligand in brain. Biochem Biophys Res Commun, 1995. 215(1): p. 89-97.

11. Lu, H.C. and K. Mackie, An Introduction to the Endogenous Cannabinoid System. Biol Psychiatry, 2016. 79(7): p. 516-25.

12. Luk, T., et al., Identification of a potent and highly efficacious, yet slowly desensitizing CB1 cannabinoid receptor agonist. Br J Pharmacol, 2004. 142(3): p. 495-500.

13. Gonsiorek, W., et al., Endocannabinoid 2-arachidonyl glycerol is a full agonist through human type 2 cannabinoid receptor: antagonism by anandamide. Mol Pharmacol, 2000. 57(5): p. 1045-50.

14. Keimpema, E., K. Mackie, and T. Harkany, Molecular model of cannabis sensitivity in developing neuronal circuits. Trends Pharmacol Sci, 2011. 32(9): p. 551-61.

15. Breivogel, C.S., et al., Cannabinoid signaling in rat cerebellar granule cells: $G$ protein activation, inhibition of glutamate release and endogenous cannabinoids. Neuropharmacology, 2004. 47(1): p. 81-91.

16. Svizenska, I., P. Dubovy, and A. Sulcova, Cannabinoid receptors 1 and 2 (CB1 and CB2), their distribution, ligands and functional involvement in nervous system structures--a short review. Pharmacol Biochem Behav, 2008. 90(4): p. 501-11.

17. Di, S., et al., Nongenomic glucocorticoid inhibition via endocannabinoid release in the hypothalamus: a fast feedback mechanism. J Neurosci, 2003. 23(12): p. 4850-7. 
18. Glass, M. and J.K. Northup, Agonist selective regulation of G proteins by cannabinoid $C B(1)$ and $C B(2)$ receptors. Mol Pharmacol, 1999. 56(6): p. 1362-9.

19. Prather, P.L., et al., Activation of cannabinoid receptors in rat brain by WIN 55212-2 produces coupling to multiple G protein alpha-subunits with different potencies. Mol Pharmacol, 2000. 57(5): p. 1000-10.

20. Jin, W., et al., Distinct domains of the CB1 cannabinoid receptor mediate desensitization and internalization. J Neurosci, 1999. 19(10): p. 3773-80.

21. Kouznetsova, M., et al., Desensitization of cannabinoid-mediated presynaptic inhibition of neurotransmission between rat hippocampal neurons in culture. Mol Pharmacol, 2002. 61(3): p. 477-85.

22. Grimsey, N.L., et al., Cannabinoid Receptor 1 trafficking and the role of the intracellular pool: implications for therapeutics. Biochem Pharmacol, 2010. 80(7): p. 1050-62.

23. Rozenfeld, R., Type I cannabinoid receptor trafficking: all roads lead to lysosome. Traffic, 2011. 12(1): p. 12-8.

24. Demuth, D.G. and A. Molleman, Cannabinoid signalling. Life Sci, 2006. 78(6): p. 549-63.

25. Rhee, M.H., et al., Cannabinoid receptor activation differentially regulates the various adenylyl cyclase isozymes. J Neurochem, 1998. 71(4): p. 1525-34.

26. Lauckner, J.E., B. Hille, and K. Mackie, The cannabinoid agonist WIN55,212-2 increases intracellular calcium via $C B 1$ receptor coupling to $G q / 11$ G proteins. Proc Natl Acad Sci U S A, 2005. 102(52): p. 19144-9.

27. Navarrete, M. and A. Araque, Endocannabinoids mediate neuron-astrocyte communication. Neuron, 2008. 57(6): p. 883-93.

28. Glass, M. and C.C. Felder, Concurrent stimulation of cannabinoid CB1 and dopamine D2 receptors augments cAMP accumulation in striatal neurons: evidence for a Gs linkage to the CB1 receptor. J Neurosci, 1997. 17(14): p. 532733.

29. Calandra, B., et al., Dual intracellular signaling pathways mediated by the human cannabinoid CB1 receptor. Eur J Pharmacol, 1999. 374(3): p. 445-55.

30. Maneuf, Y.P. and J.M. Brotchie, Paradoxical action of the cannabinoid WIN 55,212-2 in stimulated and basal cyclic AMP accumulation in rat globus pallidus slices. Br J Pharmacol, 1997. 120(8): p. 1397-8.

31. Mukhopadhyay, S., et al., The $C B(1)$ cannabinoid receptor juxtamembrane $C$ terminal peptide confers activation to specific $G$ proteins in brain. Mol Pharmacol, 2000. 57(1): p. 162-70.

32. Eldeeb, K., S. Leone-Kabler, and A.C. Howlett, CB1 cannabinoid receptormediated increases in cyclic AMP accumulation are correlated with reduced Gi/o function. J Basic Clin Physiol Pharmacol, 2016. 27(3): p. 311-22.

33. Sutherland, E.W. and T.W. Rall, Fractionation and characterization of a cyclic adenine ribonucleotide formed by tissue particles. J Biol Chem, 1958. 232(2): p. 1077-91.

34. Howlett, A.C. and R.M. Fleming, Cannabinoid inhibition of adenylate cyclase. Pharmacology of the response in neuroblastoma cell membranes. Mol Pharmacol, 1984. 26(3): p. 532-8. 
35. Sassone-Corsi, P., The cyclic AMP pathway. Cold Spring Harb Perspect Biol, 2012. 4(12).

36. Henry, D.J. and C. Chavkin, Activation of inwardly rectifying potassium channels (GIRK1) by co-expressed rat brain cannabinoid receptors in Xenopus oocytes. Neurosci Lett, 1995. 186(2-3): p. 91-4.

37. Mackie, K., et al., Cannabinoids activate an inwardly rectifying potassium conductance and inhibit Q-type calcium currents in AtT20 cells transfected with rat brain cannabinoid receptor. J Neurosci, 1995. 15(10): p. 6552-61.

38. McAllister, S.D., et al., Cannabinoid receptors can activate and inhibit G proteincoupled inwardly rectifying potassium channels in a xenopus oocyte expression system. J Pharmacol Exp Ther, 1999. 291(2): p. 618-26.

39. Azad, S.C., et al., Activation of CB1 specifically located on GABAergic interneurons inhibits LTD in the lateral amygdala. Learn Mem, 2008. 15(3): p. 143-52.

40. Guo, J. and S.R. Ikeda, Endocannabinoids modulate N-type calcium channels and G-protein-coupled inwardly rectifying potassium channels via CB1 cannabinoid receptors heterologously expressed in mammalian neurons. Mol Pharmacol, 2004. 65(3): p. 665-74.

41. May, L.M., et al., G-Protein-Coupled Inwardly Rectifying Potassium (GIRK) Channel Activation by the p75 Neurotrophin Receptor Is Required for Amyloid beta Toxicity. Front Neurosci, 2017. 11: p. 455.

42. Sugiura, T., et al., 2-Arachidonoylglycerol, a putative endogenous cannabinoid receptor ligand, induces rapid, transient elevation of intracellular free $\mathrm{Ca} 2+$ in neuroblastoma x glioma hybrid NG108-15 cells. Biochem Biophys Res Commun, 1996. 229(1): p. 58-64.

43. De Petrocellis, L., et al., Mechanisms for the coupling of cannabinoid receptors to intracellular calcium mobilization in rat insulinoma beta-cells. Exp Cell Res, 2007. 313(14): p. 2993-3004.

44. Gebremedhin, D., et al., Cannabinoid CB1 receptor of cat cerebral arterial muscle functions to inhibit L-type Ca2+ channel current. Am J Physiol, 1999. 276(6): p. H2085-93.

45. Straiker, A., et al., Cannabinoid CB1 receptors and ligands in vertebrate retina: localization and function of an endogenous signaling system. Proc Natl Acad Sci U S A, 1999. 96(25): p. 14565-70.

46. Endoh, T., Pharmacological characterization of inhibitory effects of postsynaptic opioid and cannabinoid receptors on calcium currents in neonatal rat nucleus tractus solitarius. Br J Pharmacol, 2006. 147(4): p. 391-401.

47. Rubovitch, V., M. Gafni, and Y. Sarne, The cannabinoid agonist DALN positively modulates L-type voltage-dependent calcium-channels in N18TG2 neuroblastoma cells. Brain Res Mol Brain Res, 2002. 101(1-2): p. 93-102.

48. Hampson, A.J., et al., Dual effects of anandamide on NMDA receptor-mediated responses and neurotransmission. J Neurochem, 1998. 70(2): p. 671-6.

49. Ho, B.Y., et al., Cannabinoid CB1 receptor-mediated inhibition of prolactin release and signaling mechanisms in GH4C1 cells. Endocrinology, 2000. 141(5): p. 1675-85. 
50. Brown, S.P., P.K. Safo, and W.G. Regehr, Endocannabinoids inhibit transmission at granule cell to Purkinje cell synapses by modulating three types of presynaptic calcium channels. J Neurosci, 2004. 24(24): p. 5623-31.

51. Fisyunov, A., et al., Cannabinoids modulate the P-type high-voltage-activated calcium currents in purkinje neurons. J Neurophysiol, 2006. 96(3): p. 1267-77.

52. Caulfield, M.P. and D.A. Brown, Cannabinoid receptor agonists inhibit $\mathrm{Ca}$ current in NG108-15 neuroblastoma cells via a pertussis toxin-sensitive mechanism. Br J Pharmacol, 1992. 106(2): p. 231-2.

53. Mackie, K. and B. Hille, Cannabinoids inhibit N-type calcium channels in neuroblastoma-glioma cells. Proc Natl Acad Sci U S A, 1992. 89(9): p. 3825-9.

54. Felder, C.C., et al., Anandamide, an endogenous cannabimimetic eicosanoid, binds to the cloned human cannabinoid receptor and stimulates receptormediated signal transduction. Proc Natl Acad Sci U S A, 1993. 90(16): p. 765660.

55. Mackie, K., W.A. Devane, and B. Hille, Anandamide, an endogenous cannabinoid, inhibits calcium currents as a partial agonist in N18 neuroblastoma cells. Mol Pharmacol, 1993. 44(3): p. 498-503.

56. Pan, X., S.R. Ikeda, and D.L. Lewis, Rat brain cannabinoid receptor modulates N-type Ca2+ channels in a neuronal expression system. Mol Pharmacol, 1996. 49(4): p. 707-14.

57. Freund, T.F., I. Katona, and D. Piomelli, Role of endogenous cannabinoids in synaptic signaling. Physiol Rev, 2003. 83(3): p. 1017-66.

58. Howlett, A.C., Cannabinoid receptor signaling. Handb Exp Pharmacol, 2005(168): p. 53-79.

59. Roskoski, R., Jr., ERK1/2 MAP kinases: structure, function, and regulation. Pharmacol Res, 2012. 66(2): p. 105-43.

60. Davis, M.I., J. Ronesi, and D.M. Lovinger, A predominant role for inhibition of the adenylate cyclase/protein kinase A pathway in ERK activation by cannabinoid receptor 1 in N1E-115 neuroblastoma cells. J Biol Chem, 2003. 278(49): p. 48973-80.

61. Galve-Roperh, I., et al., Mechanism of extracellular signal-regulated kinase activation by the CB(1) cannabinoid receptor. Mol Pharmacol, 2002. 62(6): p. 1385-92.

62. Korzh, A., et al., Modulation of extracellular signal-regulated kinase (ERK) by opioid and cannabinoid receptors that are expressed in the same cell. Brain Res, 2008. 1189: p. 23-32.

63. Derkinderen, P., et al., Regulation of extracellular signal-regulated kinase by cannabinoids in hippocampus. J Neurosci, 2003. 23(6): p. 2371-82.

64. Liu, J., et al., Functional CB1 cannabinoid receptors in human vascular endothelial cells. Biochem J, 2000. 346 Pt 3: p. 835-40.

65. Rueda, D., et al., The CB(1) cannabinoid receptor is coupled to the activation of c-Jun N-terminal kinase. Mol Pharmacol, 2000. 58(4): p. 814-20.

66. Mittelstadt, P.R., et al., Activating p38 MAPK: new tricks for an old kinase. Cell Cycle, 2005. 4(9): p. 1189-92.

67. Cuadrado, A. and A.R. Nebreda, Mechanisms and functions of p38 MAPK signalling. Biochem J, 2010. 429(3): p. 403-17. 
68. Keshet, Y. and R. Seger, The MAP kinase signaling cascades: a system of hundreds of components regulates a diverse array of physiological functions. Methods Mol Biol, 2010. 661: p. 3-38.

69. Bogoyevitch, M.A., et al., c-Jun N-terminal kinase (JNK) signaling: recent advances and challenges. Biochim Biophys Acta, 2010. 1804(3): p. 463-75.

70. Engstrom, W., A. Ward, and K. Moorwood, The role of scaffold proteins in JNK signalling. Cell Prolif, 2010. 43(1): p. 56-66.

71. Zarubin, T. and J. Han, Activation and signaling of the p38 MAP kinase pathway. Cell Res, 2005. 15(1): p. 11-8.

72. Downer, E.J., M.P. Fogarty, and V.A. Campbell, Tetrahydrocannabinol-induced neurotoxicity depends on CBI receptor-mediated c-Jun N-terminal kinase activation in cultured cortical neurons. Br J Pharmacol, 2003. 140(3): p. 547-57.

73. McDonald, P.H., et al., Beta-arrestin 2: a receptor-regulated MAPK scaffold for the activation of JNK3. Science, 2000. 290(5496): p. 1574-7.

74. Lefkowitz, R.J. and E.J. Whalen, beta-arrestins: traffic cops of cell signaling. Curr Opin Cell Biol, 2004. 16(2): p. 162-8.

75. Lefkowitz, R.J. and S.K. Shenoy, Transduction of receptor signals by betaarrestins. Science, 2005. 308(5721): p. 512-7.

76. Gao, H., et al., Identification of beta-arrestin2 as a $G$ protein-coupled receptorstimulated regulator of NF-kappaB pathways. Mol Cell, 2004. 14(3): p. 303-17.

77. Wang, P., et al., Beta-arrestin 2 functions as a G-protein-coupled receptoractivated regulator of oncoprotein Mdm2. J Biol Chem, 2003. 278(8): p. 6363-70.

78. Kang, J., et al., A nuclear function of beta-arrestinl in GPCR signaling: regulation of histone acetylation and gene transcription. Cell, 2005. 123(5): p. 833-47.

79. Howlett, A.C., L.C. Blume, and G.D. Dalton, $C B(1)$ cannabinoid receptors and their associated proteins. Curr Med Chem, 2010. 17(14): p. 1382-93.

80. McCudden, C.R., et al., G-protein signaling: back to the future. Cell Mol Life Sci, 2005. 62(5): p. 551-77.

81. Flores-Otero, J., et al., Ligand-specific endocytic dwell times control functional selectivity of the cannabinoid receptor 1. Nat Commun, 2014. 5: p. 4589.

82. Kenakin, T. and A. Christopoulos, Signalling bias in new drug discovery: detection, quantification and therapeutic impact. Nat Rev Drug Discov, 2013. 12(3): p. 205-16.

83. Raehal, K.M., J.K. Walker, and L.M. Bohn, Morphine side effects in beta-arrestin 2 knockout mice. J Pharmacol Exp Ther, 2005. 314(3): p. 1195-201.

84. DeWire, S.M., et al., A G protein-biased ligand at the mu-opioid receptor is potently analgesic with reduced gastrointestinal and respiratory dysfunction compared with morphine. J Pharmacol Exp Ther, 2013. 344(3): p. 708-17.

85. Manglik, A., et al., Structure-based discovery of opioid analgesics with reduced side effects. Nature, 2016. 537(7619): p. 185-190.

86. Viscusi, E.R., et al., A randomized, phase 2 study investigating TRV130, a biased ligand of the mu-opioid receptor, for the intravenous treatment of acute pain. Pain, 2016. 157(1): p. 264-72.

87. Singla, N., et al., A randomized, Phase IIb study investigating oliceridine (TRV130), a novel micro-receptor G-protein pathway selective (mu-GPS) 
modulator, for the management of moderate to severe acute pain following abdominoplasty. J Pain Res, 2017. 10: p. 2413-2424.

88. Bayewitch, M., et al., The peripheral cannabinoid receptor: adenylate cyclase inhibition and $G$ protein coupling. FEBS Lett, 1995. 375(1-2): p. 143-7.

89. Bouaboula, M., et al., Signaling pathway associated with stimulation of CB2 peripheral cannabinoid receptor. Involvement of both mitogen-activated protein kinase and induction of Krox-24 expression. Eur J Biochem, 1996. 237(3): p. 70411.

90. Zoratti, C., et al., Anandamide initiates Ca(2+) signaling via CB2 receptor linked to phospholipase $C$ in calf pulmonary endothelial cells. Br J Pharmacol, 2003. 140(8): p. 1351-62.

91. Turcotte, $\mathrm{C}$., et al., The CB2 receptor and its role as a regulator of inflammation. Cell Mol Life Sci, 2016. 73(23): p. 4449-4470.

92. Bouaboula, M., D. Dussossoy, and P. Casellas, Regulation of peripheral cannabinoid receptor CB2 phosphorylation by the inverse agonist SR 144528. Implications for receptor biological responses. J Biol Chem, 1999. 274(29): p. 20397-405.

93. Derocq, J.M., et al., Genomic and functional changes induced by the activation of the peripheral cannabinoid receptor CB2 in the promyelocytic cells $H L-60$. Possible involvement of the CB2 receptor in cell differentiation. $\mathrm{J}$ Biol Chem, 2000. 275(21): p. 15621-8.

94. Maresz, K., et al., Modulation of the cannabinoid CB2 receptor in microglial cells in response to inflammatory stimuli. J Neurochem, 2005. 95(2): p. 437-45.

95. Pertwee, R.G., et al., International Union of Basic and Clinical Pharmacology. LXXIX. Cannabinoid receptors and their ligands: beyond $C B(1)$ and $C B(2)$. Pharmacol Rev, 2010. 62(4): p. 588-631.

96. Baker, D., et al., In silico patent searching reveals a new cannabinoid receptor. Trends Pharmacol Sci, 2006. 27(1): p. 1-4.

97. Lauckner, J.E., et al., GPR55 is a cannabinoid receptor that increases intracellular calcium and inhibits M current. Proc Natl Acad Sci U S A, 2008. 105(7): p. 2699-704.

98. Balenga, N.A., et al., Pharmacology, signaling and physiological relevance of the G protein-coupled receptor 55. Adv Pharmacol, 2011. 62: p. 251-77.

99. Sawzdargo, M., et al., Identification and cloning of three novel human G proteincoupled receptor genes GPR52, PsiGPR53 and GPR55: GPR55 is extensively expressed in human brain. Brain Res Mol Brain Res, 1999. 64(2): p. 193-8.

100. Ryberg, E., et al., The orphan receptor GPR55 is a novel cannabinoid receptor. Br J Pharmacol, 2007. 152(7): p. 1092-101.

101. Henstridge, C.M., et al., The GPR55 ligand L-alpha-lysophosphatidylinositol promotes RhoA-dependent Ca2+ signaling and NFAT activation. FASEB J, 2009. 23(1): p. 183-93.

102. Henstridge, C.M., et al., GPR55 ligands promote receptor coupling to multiple signalling pathways. Br J Pharmacol, 2010. 160(3): p. 604-14.

103. Waldeck-Weiermair, M., et al., Integrin clustering enables anandamide-induced Ca2+ signaling in endothelial cells via GPR55 by protection against CB1receptor-triggered repression. J Cell Sci, 2008. 121(Pt 10): p. 1704-1717. 
104. McHugh, D., et al., Delta(9) -Tetrahydrocannabinol and N-arachidonyl glycine are full agonists at GPR18 receptors and induce migration in human endometrial HEC-1B cells. Br J Pharmacol, 2012. 165(8): p. 2414-24.

105. Kohno, M., et al., Identification of $N$-arachidonylglycine as the endogenous ligand for orphan G-protein-coupled receptor GPR18. Biochem Biophys Res Commun, 2006. 347(3): p. 827-32.

106. Console-Bram, L., et al., Activation of GPR18 by cannabinoid compounds: a tale of biased agonism. Br J Pharmacol, 2014. 171(16): p. 3908-17.

107. Gantz, I., et al., Cloning and chromosomal localization of a gene (GPR18) encoding a novel seven transmembrane receptor highly expressed in spleen and testis. Genomics, 1997. 42(3): p. 462-6.

108. Takenouchi, R., et al., N-arachidonoyl glycine induces macrophage apoptosis via GPR18. Biochem Biophys Res Commun, 2012. 418(2): p. 366-71.

109. Akopian, A.N., et al., Cannabinoids desensitize capsaicin and mustard oil responses in sensory neurons via TRPAl activation. J Neurosci, 2008. 28(5): p. 1064-75.

110. Hagenacker, T., D. Ledwig, and D. Busselberg, Feedback mechanisms in the regulation of intracellular calcium $([\mathrm{Ca} 2+] i)$ in the peripheral nociceptive system: role of TRPV-1 and pain related receptors. Cell Calcium, 2008. 43(3): p. 215-27.

111. Qin, N., et al., TRPV2 is activated by cannabidiol and mediates CGRP release in cultured rat dorsal root ganglion neurons. J Neurosci, 2008. 28(24): p. 6231-8.

112. Kim, K.Y., et al., TRP-independent inhibition of the phospholipase C pathway by natural sensory ligands. Biochem Biophys Res Commun, 2008. 370(2): p. 295300.

113. Cristino, L., et al., Immunohistochemical localization of cannabinoid type 1 and vanilloid transient receptor potential vanilloid type 1 receptors in the mouse brain. Neuroscience, 2006. 139(4): p. 1405-15.

114. Caterina, M.J., et al., A capsaicin-receptor homologue with a high threshold for noxious heat. Nature, 1999. 398(6726): p. 436-41.

115. O'Sullivan, S.E., Cannabinoids go nuclear: evidence for activation of peroxisome proliferator-activated receptors. Br J Pharmacol, 2007. 152(5): p. 576-82.

116. Sun, Y., et al., Cannabinoid activation of PPAR alpha; a novel neuroprotective mechanism. Br J Pharmacol, 2007. 152(5): p. 734-43.

117. Limbird, L.E. and R.J. Lefkowitz, Negative cooperativity among beta-adrenergic receptors in frog erythrocyte membranes. J Biol Chem, 1976. 251(16): p. 500714.

118. Milligan, G., et al., The role of GPCR dimerisation/oligomerisation in receptor signalling. Ernst Schering Found Symp Proc, 2006(2): p. 145-61.

119. Milligan, G. and N.J. Smith, Allosteric modulation of heterodimeric G-proteincoupled receptors. Trends Pharmacol Sci, 2007. 28(12): p. 615-20.

120. Bulenger, S., S. Marullo, and M. Bouvier, Emerging role of homo- and heterodimerization in G-protein-coupled receptor biosynthesis and maturation. Trends Pharmacol Sci, 2005. 26(3): p. 131-7. 
121. Waldhoer, M., et al., A heterodimer-selective agonist shows in vivo relevance of $G$ protein-coupled receptor dimers. Proc Natl Acad Sci U S A, 2005. 102(25): p. 9050-5.

122. Szidonya, L., M. Cserzo, and L. Hunyady, Dimerization and oligomerization of G-protein-coupled receptors: debated structures with established and emerging functions. J Endocrinol, 2008. 196(3): p. 435-53.

123. Wager-Miller, J., R. Westenbroek, and K. Mackie, Dimerization of G proteincoupled receptors: CB1 cannabinoid receptors as an example. Chem Phys Lipids, 2002. 121(1-2): p. 83-9.

124. Kearn, C.S., et al., Concurrent stimulation of cannabinoid CB1 and dopamine D2 receptors enhances heterodimer formation: a mechanism for receptor cross-talk? Mol Pharmacol, 2005. 67(5): p. 1697-704.

125. Carriba, P., et al., Detection of heteromerization of more than two proteins by sequential BRET-FRET. Nat Methods, 2008. 5(8): p. 727-33.

126. Rios, C., I. Gomes, and L.A. Devi, mu opioid and CB1 cannabinoid receptor interactions: reciprocal inhibition of receptor signaling and neuritogenesis. $\mathrm{Br} \mathrm{J}$ Pharmacol, 2006. 148(4): p. 387-95.

127. Jarrahian, A., V.J. Watts, and E.L. Barker, D2 dopamine receptors modulate Galpha-subunit coupling of the CB1 cannabinoid receptor. J Pharmacol Exp Ther, 2004. 308(3): p. 880-6.

128. Carriba, P., et al., Striatal adenosine A2A and cannabinoid CB1 receptors form functional heteromeric complexes that mediate the motor effects of cannabinoids. Neuropsychopharmacology, 2007. 32(11): p. 2249-59.

129. Howlett, A.C., et al., The cannabinoid receptor: biochemical, anatomical and behavioral characterization. Trends Neurosci, 1990. 13(10): p. 420-3.

130. Mackie, K., Cannabinoid receptors: where they are and what they do. J Neuroendocrinol, 2008. 20 Suppl 1: p. 10-4.

131. Castillo, P.E., et al., Endocannabinoid signaling and synaptic function. Neuron, 2012. 76(1): p. 70-81.

132. Marinelli, S., et al., The endocannabinoid 2-arachidonoylglycerol is responsible for the slow self-inhibition in neocortical interneurons. J Neurosci, 2008. 28(50): p. 13532-41.

133. Marinelli, S., et al., Self-modulation of neocortical pyramidal neurons by endocannabinoids. Nat Neurosci, 2009. 12(12): p. 1488-90.

134. Monory, K., et al., Genetic dissection of behavioural and autonomic effects of Delta(9)-tetrahydrocannabinol in mice. PLoS Biol, 2007. 5(10): p. e269.

135. Marsicano, G. and B. Lutz, Expression of the cannabinoid receptor CB1 in distinct neuronal subpopulations in the adult mouse forebrain. Eur J Neurosci, 1999. 11(12): p. 4213-25.

136. Tsou, K., et al., Cannabinoid CBI receptors are localized primarily on cholecystokinin-containing GABAergic interneurons in the rat hippocampal formation. Neuroscience, 1999. 93(3): p. 969-75.

137. Katona, I., et al., Presynaptically located CB1 cannabinoid receptors regulate GABA release from axon terminals of specific hippocampal interneurons. $\mathrm{J}$ Neurosci, 1999. 19(11): p. 4544-58. 
138. Bodor, A.L., et al., Endocannabinoid signaling in rat somatosensory cortex: laminar differences and involvement of specific interneuron types. J Neurosci, 2005. 25(29): p. 6845-56.

139. Matyas, F., et al., Subcellular localization of type 1 cannabinoid receptors in the rat basal ganglia. Neuroscience, 2006. 137(1): p. 337-61.

140. Matyas, F., et al., Molecular architecture of the cannabinoid signaling system in the core of the nucleus accumbens. Ideggyogy Sz, 2007. 60(3-4): p. 187-91.

141. Uchigashima, M., et al., Subcellular arrangement of molecules for 2arachidonoyl-glycerol-mediated retrograde signaling and its physiological contribution to synaptic modulation in the striatum. J Neurosci, 2007. 27(14): p. 3663-76.

142. Han, J., et al., Acute cannabinoids impair working memory through astroglial CB1 receptor modulation of hippocampal LTD. Cell, 2012. 148(5): p. 1039-50.

143. Molina-Holgado, E., et al., Cannabinoids promote oligodendrocyte progenitor survival: involvement of cannabinoid receptors and phosphatidylinositol-3 kinase/Akt signaling. J Neurosci, 2002. 22(22): p. 9742-53.

144. Stella, N., Endocannabinoid signaling in microglial cells. Neuropharmacology, 2009. 56 Suppl 1: p. 244-53.

145. Mackie, K., Distribution of cannabinoid receptors in the central and peripheral nervous system. Handb Exp Pharmacol, 2005(168): p. 299-325.

146. Breivogel, C.S. and S.R. Childers, The functional neuroanatomy of brain cannabinoid receptors. Neurobiol Dis, 1998. 5(6 Pt B): p. 417-31.

147. DeSanty, K.P. and M.S. Dar, Cannabinoid-induced motor incoordination through the cerebellar CB(1) receptor in mice. Pharmacol Biochem Behav, 2001. 69(1-2): p. 251-9.

148. Patel, S. and C.J. Hillard, Cannabinoid CB(1) receptor agonists produce cerebellar dysfunction in mice. J Pharmacol Exp Ther, 2001. 297(2): p. 629-37.

149. Rawls, S.M., et al., CB1 receptors in the preoptic anterior hypothalamus regulate WIN 55212-2 [(4,5-dihydro-2-methyl-4(4-morpholinylmethyl)-1-(1-naphthalenylcarbonyl)-6H-pyrr olo[3,2,1ij]quinolin-6-one]-induced hypothermia. J Pharmacol Exp Ther, 2002. 301(3): p. 963-8.

150. Fitton, A.G. and R.G. Pertwee, Changes in body temperature and oxygen consumption rate of conscious mice produced by intrahypothalamic and intracerebroventricular injections of delta 9-tetrahydrocannabinol. Br J Pharmacol, 1982. 75(2): p. 409-14.

151. Lichtman, A.H. and B.R. Martin, Spinal and supraspinal components of cannabinoid-induced antinociception. J Pharmacol Exp Ther, 1991. 258(2): p. 517-23.

152. Amici, R., et al., Sleep and bodily functions: the physiological interplay between body homeostasis and sleep homeostasis. Arch Ital Biol, 2014. 152(2-3): p. 66-78.

153. Sim, L.J., D.E. Selley, and S.R. Childers, In vitro autoradiography of receptoractivated $G$ proteins in rat brain by agonist-stimulated guanylyl 5'-[gamma[35S]thio]-triphosphate binding. Proc Natl Acad Sci U S A, 1995. 92(16): p. 7242-6. 
154. Sim-Selley, L.J., et al., Cellular localization of cannabinoid receptors and activated G-proteins in rat anterior cingulate cortex. Life Sci, 2002. 71(19): p. 2217-26.

155. Breivogel, C.S., L.J. Sim, and S.R. Childers, Regional differences in cannabinoid receptor/G-protein coupling in rat brain. J Pharmacol Exp Ther, 1997. 282(3): p. 1632-42.

156. Tam, J., et al., The cannabinoid CB1 receptor regulates bone formation by modulating adrenergic signaling. FASEB J, 2008. 22(1): p. 285-94.

157. Clapper, J.R., et al., Anandamide suppresses pain initiation through a peripheral endocannabinoid mechanism. Nat Neurosci, 2010. 13(10): p. 1265-70.

158. Price, T.J., et al., The neuronal distribution of cannabinoid receptor type 1 in the trigeminal ganglion of the rat. Neuroscience, 2003. 120(1): p. 155-62.

159. Veress, G., et al., Characterisation of cannabinoid 1 receptor expression in the perikarya, and peripheral and spinal processes of primary sensory neurons. Brain Struct Funct, 2013. 218(3): p. 733-50.

160. Izzo, A.A. and K.A. Sharkey, Cannabinoids and the gut: new developments and emerging concepts. Pharmacol Ther, 2010. 126(1): p. 21-38.

161. Miller, L.K. and L.A. Devi, The highs and lows of cannabinoid receptor expression in disease: mechanisms and their therapeutic implications. Pharmacol Rev, 2011. 63(3): p. 461-70.

162. Maccarrone, M., et al., Endocannabinoid signaling at the periphery: 50 years after THC. Trends Pharmacol Sci, 2015. 36(5): p. 277-96.

163. Montecucco, F. and V. Di Marzo, At the heart of the matter: the endocannabinoid system in cardiovascular function and dysfunction. Trends Pharmacol Sci, 2012. 33(6): p. 331-40.

164. Rozenfeld, R. and L.A. Devi, Regulation of CB1 cannabinoid receptor trafficking by the adaptor protein AP-3. FASEB J, 2008. 22(7): p. 2311-22.

165. Brailoiu, G.C., et al., Intracellular cannabinoid type 1 (CB1) receptors are activated by anandamide. J Biol Chem, 2011. 286(33): p. 29166-74.

166. Hebert-Chatelain, E., et al., Cannabinoid control of brain bioenergetics: Exploring the subcellular localization of the CB1 receptor. Mol Metab, 2014. 3(4): p. 495-504.

167. Hebert-Chatelain, E., et al., Studying mitochondrial CB1 receptors: Yes we can. Mol Metab, 2014. 3(4): p. 339.

168. Koch, M., et al., Hypothalamic POMC neurons promote cannabinoid-induced feeding. Nature, 2015. 519(7541): p. 45-50.

169. Ma, L., et al., Mitochondrial CB1 receptor is involved in ACEA-induced protective effects on neurons and mitochondrial functions. Sci Rep, 2015. 5: p. 12440.

170. Hebert-Chatelain, E., et al., A cannabinoid link between mitochondria and memory. Nature, 2016. 539(7630): p. 555-559.

171. Thibault, K., et al., Activation-dependent subcellular distribution patterns of CB1 cannabinoid receptors in the rat forebrain. Cereb Cortex, 2013. 23(11): p. 258191.

172. Mattson, M.P., M. Gleichmann, and A. Cheng, Mitochondria in neuroplasticity and neurological disorders. Neuron, 2008. 60(5): p. 748-66. 
173. Sheng, Z.H. and Q. Cai, Mitochondrial transport in neurons: impact on synaptic homeostasis and neurodegeneration. Nat Rev Neurosci, 2012. 13(2): p. 77-93.

174. Howlett, A.C., et al., International Union of Pharmacology. XXVII. Classification of cannabinoid receptors. Pharmacol Rev, 2002. 54(2): p. 161-202.

175. Tanasescu, R. and C.S. Constantinescu, Cannabinoids and the immune system: an overview. Immunobiology, 2010. 215(8): p. 588-97.

176. Liu, Y.J., et al., Cannabinoid receptor 2 suppresses leukocyte inflammatory migration by modulating the JNK/c-Jun/Alox5 pathway. J Biol Chem, 2013. 288(19): p. 13551-62.

177. Gong, J.P., et al., Cannabinoid CB2 receptors: immunohistochemical localization in rat brain. Brain Res, 2006. 1071(1): p. 10-23.

178. Walter, L., et al., Nonpsychotropic cannabinoid receptors regulate microglial cell migration. J Neurosci, 2003. 23(4): p. 1398-405.

179. Ramirez, S.H., et al., Activation of cannabinoid receptor 2 attenuates leukocyteendothelial cell interactions and blood-brain barrier dysfunction under inflammatory conditions. J Neurosci, 2012. 32(12): p. 4004-16.

180. Van Sickle, M.D., et al., Identification and functional characterization of brainstem cannabinoid CB2 receptors. Science, 2005. 310(5746): p. 329-32.

181. Viscomi, M.T., et al., Selective CB2 receptor agonism protects central neurons from remote axotomy-induced apoptosis through the PI3K/Akt pathway. $\mathrm{J}$ Neurosci, 2009. 29(14): p. 4564-70.

182. Dhopeshwarkar, A. and K. Mackie, CB2 Cannabinoid receptors as a therapeutic target-what does the future hold? Mol Pharmacol, 2014. 86(4): p. 430-7.

183. Atwood, B.K. and K. Mackie, CB2: a cannabinoid receptor with an identity crisis. Br J Pharmacol, 2010. 160(3): p. 467-79.

184. den Boon, F.S., et al., Excitability of prefrontal cortical pyramidal neurons is modulated by activation of intracellular type-2 cannabinoid receptors. Proc Natl Acad Sci U S A, 2012. 109(9): p. 3534-9.

185. McCance-Katz, E.F., The Substance Abuse and Mental Health Services Administration (SAMHSA): New Directions. Psychiatr Serv, 2018. 69(10): p. 1046-1048.

186. Carliner, H., et al., Cannabis use, attitudes, and legal status in the U.S.: A review. Prev Med, 2017. 104: p. 13-23.

187. ElSohly, M.A., et al., Changes in Cannabis Potency Over the Last 2 Decades (1995-2014): Analysis of Current Data in the United States. Biol Psychiatry, 2016. 79(7): p. 613-9.

188. Arora, K., et al., Measuring Attitudes Toward Medical and Recreational Cannabis Among Older Adults in Colorado. Gerontologist, 2019.

189. Felson, J., A. Adamczyk, and C. Thomas, How and why have attitudes about cannabis legalization changed so much? Soc Sci Res, 2019. 78: p. 12-27.

190. Miech, R.A., et al., Trends in use of marijuana and attitudes toward marijuana among youth before and after decriminalization: the case of California 20072013. Int J Drug Policy, 2015. 26(4): p. 336-44.

191. Pacula, R.L., M. Jacobson, and E.J. Maksabedian, In the weeds: a baseline view of cannabis use among legalizing states and their neighbours. Addiction, 2016. 111(6): p. 973-80. 
192. Breivogel, C.S. and L.J. Sim-Selley, Basic neuroanatomy and neuropharmacology of cannabinoids. Int Rev Psychiatry, 2009. 21(2): p. 113-21.

193. Pacher, P., S. Batkai, and G. Kunos, The endocannabinoid system as an emerging target of pharmacotherapy. Pharmacol Rev, 2006. 58(3): p. 389-462.

194. Iversen, L., Cannabis and the brain. Brain, 2003. 126(Pt 6): p. 1252-70.

195. Fine, P.G. and M.J. Rosenfeld, The endocannabinoid system, cannabinoids, and pain. Rambam Maimonides Med J, 2013. 4(4): p. e0022.

196. Donvito, G., et al., The Endogenous Cannabinoid System: A Budding Source of Targets for Treating Inflammatory and Neuropathic Pain. Neuropsychopharmacology, 2018. 43(1): p. 52-79.

197. Jhaveri, M.D., et al., Cannabinoid CB2 receptor-mediated anti-nociception in models of acute and chronic pain. Mol Neurobiol, 2007. 36(1): p. 26-35.

198. Chiarlone, A., et al., A restricted population of CB1 cannabinoid receptors with neuroprotective activity. Proc Natl Acad Sci U S A, 2014. 111(22): p. 8257-62.

199. Marsicano, G., et al., CB1 cannabinoid receptors and on-demand defense against excitotoxicity. Science, 2003. 302(5642): p. 84-8.

200. Kim, S.H., et al., Molecular mechanisms of cannabinoid protection from neuronal excitotoxicity. Mol Pharmacol, 2006. 69(3): p. 691-6.

201. Khaspekov, L.G., et al., Involvement of brain-derived neurotrophic factor in cannabinoid receptor-dependent protection against excitotoxicity. Eur J Neurosci, 2004. 19(7): p. 1691-8.

202. Haghani, M., et al., CB1 cannabinoid receptor activation rescues amyloid betainduced alterations in behaviour and intrinsic electrophysiological properties of rat hippocampal CA1 pyramidal neurones. Cell Physiol Biochem, 2012. 29(3-4): p. 391-406.

203. Aso, E., et al., CB1 agonist ACEA protects neurons and reduces the cognitive impairment of AbetaPP/PS1 mice. J Alzheimers Dis, 2012. 30(2): p. 439-59.

204. Milton, N.G., Anandamide and noladin ether prevent neurotoxicity of the human amyloid-beta peptide. Neurosci Lett, 2002. 332(2): p. 127-30.

205. Westlake, T.M., et al., Cannabinoid receptor binding and messenger RNA expression in human brain: an in vitro receptor autoradiography and in situ hybridization histochemistry study of normal aged and Alzheimer's brains. Neuroscience, 1994. 63(3): p. 637-52.

206. Ramirez, B.G., et al., Prevention of Alzheimer's disease pathology by cannabinoids: neuroprotection mediated by blockade of microglial activation. $\mathrm{J}$ Neurosci, 2005. 25(8): p. 1904-13.

207. van der Stelt, M., et al., Endocannabinoids and beta-amyloid-induced neurotoxicity in vivo: effect of pharmacological elevation of endocannabinoid levels. Cell Mol Life Sci, 2006. 63(12): p. 1410-24.

208. Glass, M., M. Dragunow, and R.L. Faull, The pattern of neurodegeneration in Huntington's disease: a comparative study of cannabinoid, dopamine, adenosine and $G A B A(A)$ receptor alterations in the human basal ganglia in Huntington's disease. Neuroscience, 2000. 97(3): p. 505-19.

209. Blazquez, C., et al., Loss of striatal type 1 cannabinoid receptors is a key pathogenic factor in Huntington's disease. Brain, 2011. 134(Pt 1): p. 119-36. 
210. Steffens, S. and P. Pacher, Targeting cannabinoid receptor $C B(2)$ in cardiovascular disorders: promises and controversies. Br J Pharmacol, 2012. 167(2): p. 313-23.

211. Di Marzo, V. and I. Matias, Endocannabinoid control of food intake and energy balance. Nat Neurosci, 2005. 8(5): p. 585-9.

212. Kirkham, T.C., et al., Endocannabinoid levels in rat limbic forebrain and hypothalamus in relation to fasting, feeding and satiation: stimulation of eating by 2-arachidonoyl glycerol. Br J Pharmacol, 2002. 136(4): p. 550-7.

213. Di Marzo, V., Targeting the endocannabinoid system: to enhance or reduce? Nat Rev Drug Discov, 2008. 7(5): p. 438-55.

214. Volkow, N.D., et al., Adverse health effects of marijuana use. N Engl J Med, 2014. 370(23): p. 2219-27.

215. Di Marzo, V., N. Stella, and A. Zimmer, Endocannabinoid signalling and the deteriorating brain. Nat Rev Neurosci, 2015. 16(1): p. 30-42.

216. Volkow, N.D., W.M. Compton, and S.R. Weiss, Adverse health effects of marijuana use. N Engl J Med, 2014. 371(9): p. 879.

217. Ishiguro, H., et al., Brain cannabinoid CB2 receptor in schizophrenia. Biol Psychiatry, 2010. 67(10): p. 974-82.

218. Bae, J.S., et al., Genetic association analysis of CNR1 and CNR2 polymorphisms with schizophrenia in a Korean population. Psychiatr Genet, 2014. 24(5): p. 2259.

219. Ujike, H., et al., CNR1, central cannabinoid receptor gene, associated with susceptibility to hebephrenic schizophrenia. Mol Psychiatry, 2002. 7(5): p. 515-8.

220. Norrod, A.G. and R.A. Puffenbarger, Genetic polymorphisms of the endocannabinoid system. Chem Biodivers, 2007. 4(8): p. 1926-32.

221. Schlossarek, S., et al., Psychosocial Determinants of Cannabis Dependence: A Systematic Review of the Literature. Eur Addict Res, 2016. 22(3): p. 131-44.

222. Lopez-Quintero, C., et al., Probability and predictors of transition from first use to dependence on nicotine, alcohol, cannabis, and cocaine: results of the National Epidemiologic Survey on Alcohol and Related Conditions (NESARC). Drug Alcohol Depend, 2011. 115(1-2): p. 120-30.

223. Hall, W. and L. Degenhardt, Adverse health effects of non-medical cannabis use. Lancet, 2009. 374(9698): p. 1383-91.

224. Wright, S. and J. Metts, Recreational cannabinoid use: The hazards behind the "high". J Fam Pract, 2016. 65(11): p. 770-779.

225. Hanson, C.L., et al., An exploration of social circles and prescription drug abuse through Twitter. J Med Internet Res, 2013. 15(9): p. e189.

226. Kendler, K.S., et al., A developmental etiological model for drug abuse in men. Drug Alcohol Depend, 2017. 179: p. 220-228.

227. de Wit, H. and T.J. Phillips, Do initial responses to drugs predict future use or abuse? Neurosci Biobehav Rev, 2012. 36(6): p. 1565-76.

228. Bieber, C.M., et al., Retrospective accounts of initial subjective effects of opioids in patients treated for pain who do or do not develop opioid addiction: a pilot case-control study. Exp Clin Psychopharmacol, 2008. 16(5): p. 429-34.

229. Griffiths, R.R. and P.P. Woodson, Reinforcing effects of caffeine in humans. J Pharmacol Exp Ther, 1988. 246(1): p. 21-9. 
230. Lambert, N.M., M. McLeod, and S. Schenk, Subjective responses to initial experience with cocaine: an exploration of the incentive-sensitization theory of drug abuse. Addiction, 2006. 101(5): p. 713-25.

231. Davidson, E.S. and S. Schenk, Variability in subjective responses to marijuana: initial experiences of college students. Addict Behav, 1994. 19(5): p. 531-8.

232. Le Strat, Y., et al., First positive reactions to cannabis constitute a priority risk factor for cannabis dependence. Addiction, 2009. 104(10): p. 1710-7.

233. Nishida, K.S., et al., Individual differences in initial morphine sensitivity as a predictor for the development of opiate addiction in rats. Behav Brain Res, 2016. 313: p. 315-323.

234. Shen, E.H., et al., Correlated responses to selection in FAST and SLOW mice: effects of ethanol on ataxia, temperature, sedation, and withdrawal. Alcohol Clin Exp Res, 1996. 20(4): p. 688-96.

235. Phillips, T.J., et al., Forward, relaxed, and reverse selection for reduced and enhanced sensitivity to ethanol's locomotor stimulant effects in mice. Alcohol Clin Exp Res, 2002. 26(5): p. 593-602.

236. Ramsay, D.S., et al., Predicting addictive vulnerability: individual differences in initial responding to a drug's pharmacological effects. PLoS One, 2015. 10(4): p. e0124740.

237. Schuckit, M.A., Low level of response to alcohol as a predictor of future alcoholism. Am J Psychiatry, 1994. 151(2): p. 184-9.

238. Quinn, P.D. and K. Fromme, Subjective response to alcohol challenge: $a$ quantitative review. Alcohol Clin Exp Res, 2011. 35(10): p. 1759-70.

239. Schuckit, M.A., An overview of genetic influences in alcoholism. J Subst Abuse Treat, 2009. 36(1): p. S5-14.

240. Kreek, M.J., et al., Opiate addiction and cocaine addiction: underlying molecular neurobiology and genetics. J Clin Invest, 2012. 122(10): p. 3387-93.

241. Perez-Rubio, G., et al., Nicotine Addiction Development: From Epidemiology to Genetic Factors. Rev Invest Clin, 2015. 67(6): p. 333-43.

242. Kendler, K.S., et al., Genetic and environmental influences on alcohol, caffeine, cannabis, and nicotine use from early adolescence to middle adulthood. Arch Gen Psychiatry, 2008. 65(6): p. 674-82.

243. Agrawal, A. and M.T. Lynskey, The genetic epidemiology of cannabis use, abuse and dependence. Addiction, 2006. 101(6): p. 801-12.

244. Han, S., et al., Linkage analysis followed by association show NRG1 associated with cannabis dependence in African Americans. Biol Psychiatry, 2012. 72(8): p. 637-44.

245. Sherva, R., et al., Genome-wide Association Study of Cannabis Dependence Severity, Novel Risk Variants, and Shared Genetic Risks. JAMA Psychiatry, 2016. 73(5): p. 472-80.

246. Stringer, S., et al., Genome-wide association study of lifetime cannabis use based on a large meta-analytic sample of 32330 subjects from the International Cannabis Consortium. Transl Psychiatry, 2016. 6: p. e769.

247. Pasman, J.A., et al., GWAS of lifetime cannabis use reveals new risk loci, genetic overlap with psychiatric traits, and a causal influence of schizophrenia. Nat Neurosci, 2018. 21(9): p. 1161-1170. 
248. Brezing, C.A. and F.R. Levin, The Current State of Pharmacological Treatments for Cannabis Use Disorder and Withdrawal. Neuropsychopharmacology, 2018. 43(1): p. 173-194.

249. Green, B., D. Kavanagh, and R. Young, Being stoned: a review of self-reported cannabis effects. Drug Alcohol Rev, 2003. 22(4): p. 453-60.

250. Sewell, R.A., et al., Acute effects of THC on time perception in frequent and infrequent cannabis users. Psychopharmacology (Berl), 2013. 226(2): p. 401-13.

251. Freeman, D., et al., How cannabis causes paranoia: using the intravenous administration of 9-tetrahydrocannabinol (THC) to identify key cognitive mechanisms leading to paranoia. Schizophr Bull, 2015. 41(2): p. 391-9.

252. Lichtman, A.H., et al., The pharmacological activity of inhalation exposure to marijuana smoke in mice. Drug Alcohol Depend, 2001. 63(2): p. 107-16.

253. Fan, F., et al., Development of cross-tolerance between delta 9tetrahydrocannabinol, CP 55,940 and WIN 55,212. J Pharmacol Exp Ther, 1994. 271(3): p. 1383-90.

254. Hayakawa, K., et al., Cannabidiol potentiates pharmacological effects of Delta(9)-tetrahydrocannabinol via $C B(1)$ receptor-dependent mechanism. Brain Res, 2008. 1188: p. 157-64.

255. Marshell, R., et al., In vivo effects of synthetic cannabinoids JWH-018 and JWH073 and phytocannabinoid Delta9-THC in mice: inhalation versus intraperitoneal injection. Pharmacol Biochem Behav, 2014. 124: p. 40-7.

256. Bass, C.E. and B.R. Martin, Time course for the induction and maintenance of tolerance to Delta(9)-tetrahydrocannabinol in mice. Drug Alcohol Depend, 2000. 60(2): p. 113-9.

257. Kasten, C.R., Y. Zhang, and S.L. Boehm, 2nd, Acute and long-term effects of Delta9-tetrahydrocannabinol on object recognition and anxiety-like activity are age- and strain-dependent in mice. Pharmacol Biochem Behav, 2017. 163: p. 919.

258. Kasten, C.R., Y. Zhang, and S.L. Boehm, 2nd, Acute Cannabinoids Produce Robust Anxiety-Like and Locomotor Effects in Mice, but Long-Term Consequences Are Age- and Sex-Dependent. Front Behav Neurosci, 2019. 13: p. 32.

259. Long, L.E., et al., A behavioural comparison of acute and chronic Delta9tetrahydrocannabinol and cannabidiol in C57BL/6JArc mice. Int J Neuropsychopharmacol, 2010. 13(7): p. 861-76.

260. McMahon, L.R. and W. Koek, Differences in the relative potency of SR 141716A and AM 251 as antagonists of various in vivo effects of cannabinoid agonists in C57BL/6J mice. Eur J Pharmacol, 2007. 569(1-2): p. 70-6.

261. Onaivi, E.S., et al., Neurobehavioral effects of delta 9-THC and cannabinoid (CB1) receptor gene expression in mice. Behav Brain Res, 1995. 72(1-2): p. 11525.

262. Wang, X., et al., Joint mouse-human phenome-wide association to test gene function and disease risk. Nat Commun, 2016. 7: p. 10464.

263. Andreux, P.A., et al., Systems genetics of metabolism: the use of the BXD murine reference panel for multiscalar integration of traits. Cell, 2012. 150(6): p. 128799. 
264. Grisel, J.E., et al., Quantitative trait loci affecting methamphetamine responses in BXD recombinant inbred mouse strains. J Neurosci, 1997. 17(2): p. 745-54.

265. Philip, V.M., et al., High-throughput behavioral phenotyping in the expanded panel of BXD recombinant inbred strains. Genes Brain Behav, 2010. 9(2): p. 12959.

266. Dickson, P.E., et al., Systems genetics of intravenous cocaine self-administration in the BXD recombinant inbred mouse panel. Psychopharmacology (Berl), 2016. 233(4): p. 701-14.

267. Harenza, J.L., et al., Genetic variation within the Chrna7 gene modulates nicotine reward-like phenotypes in mice. Genes Brain Behav, 2014. 13(2): p. 213-25.

268. Mulligan, M.K., et al., Genetic Contribution to Initial and Progressive Alcohol Intake Among Recombinant Inbred Strains of Mice. Front Genet, 2018. 9: p. 370.

269. Williams, R.W. and E.G. Williams, Resources for Systems Genetics. Methods Mol Biol, 2017. 1488: p. 3-29.

270. Compton, D.R., et al., In vivo characterization of a specific cannabinoid receptor antagonist (SR141716A): inhibition of delta 9-tetrahydrocannabinol-induced responses and apparent agonist activity. J Pharmacol Exp Ther, 1996. 277(2): p. 586-94.

271. Huestis, M.A., et al., Blockade of effects of smoked marijuana by the CB1selective cannabinoid receptor antagonist SR141716. Arch Gen Psychiatry, 2001. 58(4): p. 322-8.

272. Ledent, C., et al., Unresponsiveness to cannabinoids and reduced addictive effects of opiates in CB1 receptor knockout mice. Science, 1999. 283(5400): p. 401-4.

273. Rinaldi-Carmona, M., et al., SR141716A, a potent and selective antagonist of the brain cannabinoid receptor. FEBS Lett, 1994. 350(2-3): p. 240-4.

274. Zimmer, A., et al., Increased mortality, hypoactivity, and hypoalgesia in cannabinoid CB1 receptor knockout mice. Proc Natl Acad Sci U S A, 1999. 96(10): p. 5780-5.

275. Parks, C., et al., Sex and Strain Variation in Initial Sensitivity and Rapid Tolerance to D9-Tetrahydrocannabinol. Cannabis and Cannabinoid Research, 2020. 5(3).

276. Clarkson, J.M., et al., Handling method alters the hedonic value of reward in laboratory mice. Sci Rep, 2018. 8(1): p. 2448.

277. Gouveia, K. and J.L. Hurst, Optimising reliability of mouse performance in behavioural testing: the major role of non-aversive handling. Sci Rep, 2017. 7: p. 44999.

278. Hurst, J.L. and R.S. West, Taming anxiety in laboratory mice. Nat Methods, 2010. 7(10): p. 825-6.

279. Mulligan, M.K., et al., Impact of Genetic Variation on Stress-Related Ethanol Consumption. Alcohol Clin Exp Res, 2019. 43(7): p. 1391-1402.

280. Kasten, C.R., et al., Short-Term Genetic Selection for Adolescent Locomotor Sensitivity to Delta9-Tetrahydrocannabinol (THC). Behav Genet, 2018. 48(3): p. 224-235.

281. Parks, C., et al., Comparison and Functional Genetic Analysis of Striatal Protein Expression Among Diverse Inbred Mouse Strains. Front Mol Neurosci, 2019. 12: p. 128. 
282. Sim-Selley, L.J., Regulation of cannabinoid CB1 receptors in the central nervous system by chronic cannabinoids. Crit Rev Neurobiol, 2003. 15(2): p. 91-119.

283. Sim-Selley, L.J., et al., Prolonged recovery rate of CB1 receptor adaptation after cessation of long-term cannabinoid administration. Mol Pharmacol, 2006. 70(3): p. 986-96.

284. Hirvonen, J., et al., Reversible and regionally selective downregulation of brain cannabinoid CB1 receptors in chronic daily cannabis smokers. Mol Psychiatry, 2012. 17(6): p. 642-9.

285. McKinney, D.L., et al., Dose-related differences in the regional pattern of cannabinoid receptor adaptation and in vivo tolerance development to delta9tetrahydrocannabinol. J Pharmacol Exp Ther, 2008. 324(2): p. 664-73.

286. Cooper, Z.D. and R.M. Craft, Sex-Dependent Effects of Cannabis and Cannabinoids: A Translational Perspective. Neuropsychopharmacology, 2018. 43(1): p. 34-51.

287. Schlienz, N.J., et al., Cannabis Withdrawal: A Review of Neurobiological Mechanisms and Sex Differences. Curr Addict Rep, 2017. 4(2): p. 75-81.

288. Cooper, Z.D. and M. Haney, Investigation of sex-dependent effects of cannabis in daily cannabis smokers. Drug Alcohol Depend, 2014. 136: p. 85-91.

289. Cooper, Z.D. and M. Haney, Sex-dependent effects of cannabis-induced analgesia. Drug Alcohol Depend, 2016. 167: p. 112-20.

290. Craft, R.M., R. Kandasamy, and S.M. Davis, Sex differences in anti-allodynic, anti-hyperalgesic and anti-edema effects of Delta(9)-tetrahydrocannabinol in the rat. Pain, 2013. 154(9): p. 1709-17.

291. Craft, R.M., J.A. Marusich, and J.L. Wiley, Sex differences in cannabinoid pharmacology: a reflection of differences in the endocannabinoid system? Life Sci, 2013. 92(8-9): p. 476-81.

292. Stinson, F.S., et al., Cannabis use disorders in the USA: prevalence, correlates and co-morbidity. Psychol Med, 2006. 36(10): p. 1447-60.

293. Nguyen, J.D., et al., Tolerance to hypothermic and antinoceptive effects of 9tetrahydrocannabinol (THC) vapor inhalation in rats. Pharmacol Biochem Behav, 2018. 172: p. 33-38.

294. Marusich, J.A., et al., The impact of gonadal hormones on cannabinoid dependence. Exp Clin Psychopharmacol, 2015. 23(4): p. 206-16.

295. Delprato, A., et al., Systems genetic analysis of hippocampal neuroanatomy and spatial learning in mice. Genes Brain Behav, 2015. 14(8): p. 591-606.

296. Graybeal, C., et al., Strains and stressors: an analysis of touchscreen learning in genetically diverse mouse strains. PLoS One, 2014. 9(2): p. e87745.

297. Knoll, A.T., et al., Quantitative Trait Loci and a Novel Genetic Candidate for Fear Learning. J Neurosci, 2016. 36(23): p. 6258-68.

298. Laughlin, R.E., et al., Genetic dissection of behavioral flexibility: reversal learning in mice. Biol Psychiatry, 2011. 69(11): p. 1109-16.

299. Neuner, S.M., et al., Systems genetics identifies Hplbp3 as a novel modulator of cognitive aging. Neurobiol Aging, 2016. 46: p. 58-67.

300. Owen, E.H., et al., Identification of quantitative trait loci involved in contextual and auditory-cued fear conditioning in BXD recombinant inbred strains. Behav Neurosci, 1997. 111(2): p. 292-300. 
301. Shea, C.J., et al., Variable impact of chronic stress on spatial learning and memory in BXD mice. Physiol Behav, 2015. 150: p. 69-77.

302. Brigman, J.L., et al., Genetic relationship between anxiety-related and fearrelated behaviors in BXD recombinant inbred mice. Behav Pharmacol, 2009. 20(2): p. 204-9.

303. Carhuatanta, K.A., et al., Unique genetic loci identified for emotional behavior in control and chronic stress conditions. Front Behav Neurosci, 2014. 8: p. 341.

304. Loos, M., et al., Independent genetic loci for sensorimotor gating and attentional performance in BXD recombinant inbred strains. Genes Brain Behav, 2012. 11(2): p. 147-56.

305. Hitzemann, R., et al., Dopamine D2 receptor binding, Drd2 expression and the number of dopamine neurons in the BXD recombinant inbred series: genetic relationships to alcohol and other drug associated phenotypes. Alcohol Clin Exp Res, 2003. 27(1): p. 1-11.

306. Rodriguez, L.A., et al., Alcohol acceptance, preference, and sensitivity in mice. I. Quantitative genetic analysis using BXD recombinant inbred strains. Alcohol Clin Exp Res, 1994. 18(6): p. 1416-22.

307. Rodriguez, L.A., et al., Alcohol acceptance, preference, and sensitivity in mice. II. Quantitative trait loci mapping analysis using BXD recombinant inbred strains. Alcohol Clin Exp Res, 1995. 19(2): p. 367-73.

308. Phillips, T.J., J.K. Belknap, and J.C. Crabbe, Use of recombinant inbred strains to assess vulnerability to drug abuse at the genetic level. J Addict Dis, 1991. 10(12): p. 73-87.

309. Jackson, K.J., et al., The neuropeptide galanin and variants in the GalRl gene are associated with nicotine dependence. Neuropsychopharmacology, 2011. 36(11): p. 2339-48.

310. Ashbrook, D., et al., The expanded BXD family of mice: A cohort for experimental systems genetics and precision medicine. bioRxiv, 2019.

311. Newbury, A.J. and G.D. Rosen, Genetic, morphometric, and behavioral factors linked to the midsagittal area of the corpus callosum. Front Genet, 2012. 3: p. 91.

312. Knoll, A.T., K. Jiang, and P. Levitt, Quantitative trait locus mapping and analysis of heritable variation in affiliative social behavior and co-occurring traits. Genes Brain Behav, 2018. 17(5): p. e12431.

313. Loos, M., et al., Neuregulin-3 in the mouse medial prefrontal cortex regulates impulsive action. Biol Psychiatry, 2014. 76(8): p. 648-55.

314. Parker, C.C., et al., Systems Genetic Analysis in GeneNetwork.org. Curr Protoc Neurosci, 2017. 79: p. 839 1-8 3920.

315. Mulligan, M.K., et al., GeneNetwork: A Toolbox for Systems Genetics. Methods Mol Biol, 2017. 1488: p. 75-120.

316. Svenson, K.L., et al., High-resolution genetic mapping using the Mouse Diversity outbred population. Genetics, 2012. 190(2): p. 437-47.

317. Threadgill, D.W., et al., The collaborative cross: a recombinant inbred mouse population for the systems genetic era. ILAR J, 2011. 52(1): p. 24-31.

318. Lazenka, M.F., D.E. Selley, and L.J. Sim-Selley, Brain regional differences in CB1 receptor adaptation and regulation of transcription. Life Sci, 2013. 92(8-9): p. 446-52. 
319. Hill, W. and T. Mackay, D.S. Falconer and Introduction to Quantitative Genetics. Genetics, 2004. 167(4): p. 1529-1536.

320. Lariviere, W.R. and J.S. Mogil, The genetics of pain and analgesia in laboratory animals. Methods Mol Biol, 2010. 617: p. 261-78.

321. Zhou, X. and M. Stephens, Efficient multivariate linear mixed model algorithms for genome-wide association studies. Nat Methods, 2014. 11(4): p. 407-9.

322. Chen, H. and B.M. Sharp, Content-rich biological network constructed by mining PubMed abstracts. BMC Bioinformatics, 2004. 5: p. 147.

323. Puighermanal, E., et al., Cannabinoid modulation of hippocampal long-term memory is mediated by mTOR signaling. Nat Neurosci, 2009. 12(9): p. 1152-8.

324. Nestler, E.J., The neurobiology of cocaine addiction. Sci Pract Perspect, 2005. 3(1): p. 4-10.

325. Ferland, J.N. and Y.L. Hurd, Deconstructing the neurobiology of cannabis use disorder. Nat Neurosci, 2020. 23(5): p. 600-610.

326. Cwetsch, A.W., et al., In vivo methods for acute modulation of gene expression in the central nervous system. Prog Neurobiol, 2018. 168: p. 69-85.

327. Torres-Ruiz, R. and S. Rodriguez-Perales, CRISPR-Cas9 technology: applications and human disease modelling. Brief Funct Genomics, 2017. 16(1): p. 4-12.

328. Mulligan, M.K., et al., Identification of a Functional Non-coding Variant in the GABA A Receptor alpha2 Subunit of the C57BL/6J Mouse Reference Genome: Major Implications for Neuroscience Research. Front Genet, 2019. 10: p. 188.

329. Sharma, P., P. Murthy, and M.M. Bharath, Chemistry, metabolism, and toxicology of cannabis: clinical implications. Iran J Psychiatry, 2012. 7(4): p. 149-56.

330. Huestis, M.A., J.E. Henningfield, and E.J. Cone, Blood cannabinoids. I. Absorption of THC and formation of 11-OH-THC and THCCOOH during and after smoking marijuana. J Anal Toxicol, 1992. 16(5): p. 276-82.

331. Abrams, D.I. and M. Guzman, Cannabis in cancer care. Clin Pharmacol Ther, 2015. 97(6): p. 575-86.

332. Chang, E., et al., CB1 receptor blockade ameliorates hepatic fat infiltration and inflammation and increases Nrf2-AMPK pathway in a rat model of severely uncontrolled diabetes. PLoS One, 2018. 13(10): p. e0206152.

333. O'Sullivan, S.E., Endocannabinoids and the Cardiovascular System in Health and Disease. Handb Exp Pharmacol, 2015. 231: p. 393-422.

334. Liedhegner, E. S., C. D. Vogt, D. S. Sem, C. W. Cunningham, and C. J. Hillard. 2014. 'Sterol carrier protein-2: binding protein for endocannabinoids', Mol Neurobiol, 50: 149-58.

335. Martin, G., et al. 2019. 'Sterol Carrier Protein-2/Sterol Carrier Protein-x/Fatty Acid Binding Protein-1 Ablation Impacts Response of Brain Endocannabinoid to High-Fat Diet', Lipids, 54: 583-601.

336. Puighermanal, E., A. Busquets-Garcia, M. Gomis-Gonzalez, G. Marsicano, R. Maldonado, and A. Ozaita. 2013. 'Dissociation of the pharmacological effects of THC by mTOR blockade', Neuropsychopharmacology, 38: 1334-43.

337. Cherry, J. A., and R. L. Davis. 1999. 'Cyclic AMP phosphodiesterases are localized in regions of the mouse brain associated with reinforcement, movement, and affect', J Comp Neurol, 407: 287-301. 
338. Nestler, E. J. 2016. 'Reflections on: "A general role for adaptations in G-Proteins and the cyclic AMP system in mediating the chronic actions of morphine and cocaine on neuronal function"', Brain Res, 1645: 71-4.

339. Perez-Torres, S. et al.. 2000. 'Phosphodiesterase type 4 isozymes expression in human brain examined by in situ hybridization histochemistry and[3H]rolipram binding autoradiography. Comparison with monkey and rat brain', J Chem Neuroanat, 20: 349-74.

340. Muschamp, J. W., and W. A. Carlezon, Jr. 2013. 'Roles of nucleus accumbens CREB and dynorphin in dysregulation of motivation', Cold Spring Harb Perspect Med, 3: a012005.

341. Olsen, C. M., and Q. S. Liu. 2016. 'Phosphodiesterase 4 inhibitors and drugs of abuse: current knowledge and therapeutic opportunities', Front Biol (Beijing), 11: 376-86.

342. Ji, S. et al. 2006. 'Disruption of PTEN coupling with 5-HT2C receptors suppresses behavioral responses induced by drugs of abuse', Nat Med, 12: 324-9.

343. Maillet, J. C., Y. Zhang, X. Li, and X. Zhang. 2008. 'PTEN-5-HT2C coupling: a new target for treating drug addiction', Prog Brain Res, 172: 407-20.

344. Demontis, et al. 2019. 'Genome-wide association study implicates CHRNA2 in cannabis use disorder', Nat Neurosci, 22: 1066-74.

345. Boucher, A. et al. 2007. 'Heterozygous neuregulin 1 mice are more sensitive to the behavioural effects of Delta9-tetrahydrocannabinol', Psychopharmacology (Berl), 192: 325-36.

346. Boucher, A.et al. 2011. 'The schizophrenia susceptibility gene neuregulin 1 modulates tolerance to the effects of cannabinoids', Int J Neuropsychopharmacol, 14: 631-43.

347. Newell, K. A., T. Karl, and X. F. Huang. 2013. 'A neuregulin 1 transmembrane domain mutation causes imbalanced glutamatergic and dopaminergic receptor expression in mice', Neuroscience, 248: 670-80.

348. Parsons, L. H., and Y. L. Hurd. 2015. 'Endocannabinoid signalling in reward and addiction', Nat Rev Neurosci, 16: 579-94.

349. Alvarez-Jaimes, L., I. Polis, and L. H. Parsons. 2009. 'Regional Influence of Cannabinoid CB1 Receptors in the Regulation of Ethanol Self-Administration by Wistar Rats', Open Neuropsychopharmacol J, 2: 77-85.

350. Alvarez-Jaimes, L., D. G. Stouffer, and L. H. Parsons. 2009. 'Chronic ethanol treatment potentiates ethanol-induced increases in interstitial nucleus accumbens endocannabinoid levels in rats', J Neurochem, 111: 37-48.

351. Caille, S. et al. 2007. 'Specific alterations of extracellular endocannabinoid levels in the nucleus accumbens by ethanol, heroin, and cocaine self-administration', $J$ Neurosci, 27: 3695-702.

352. Ceccarini, J., C. Casteels, M. Koole, G. Bormans, and K. Van Laere. 2013. 'Transient changes in the endocannabinoid system after acute and chronic ethanol exposure and abstinence in the rat: a combined PET and microdialysis study', Eur J Nucl Med Mol Imaging, 40: 1582-94.

353. Ellgren, M., S. M. Spano, and Y. L. Hurd. 2007. 'Adolescent cannabis exposure alters opiate intake and opioid limbic neuronal populations in adult rats', Neuropsychopharmacology, 32: 607-15. 
354. Panagis, G., B. Mackey, and S. Vlachou. 2014. 'Cannabinoid Regulation of Brain Reward Processing with an Emphasis on the Role of CB1 Receptors: A Step Back into the Future', Front Psychiatry, 5: 92.

355. Serrano, A., and L. H. Parsons. 2011. 'Endocannabinoid influence in drug reinforcement, dependence and addiction-related behaviors', Pharmacol Ther, 132: 215-41.

356. Grueter, B. A. et al. 2006. 'Extracellular-signal regulated kinase 1-dependent metabotropic glutamate receptor 5-induced long-term depression in the bed nucleus of the stria terminalis is disrupted by cocaine administration', J Neurosci, 26: 3210-9.

357. Liu, Q. S., L. Pu, and M. M. Poo. 2005. 'Repeated cocaine exposure in vivo facilitates LTP induction in midbrain dopamine neurons', Nature, 437: 1027-31.

358. Pan, B., C. J. Hillard, and Q. S. Liu. 2008. 'Endocannabinoid signaling mediates cocaine-induced inhibitory synaptic plasticity in midbrain dopamine neurons', $J$ Neurosci, 28: 1385-97.

359. Fourgeaud, L., S. Mato, D. Bouchet, A. Hemar, P. F. Worley, and O. J. Manzoni. 2004. 'A single in vivo exposure to cocaine abolishes endocannabinoid-mediated long-term depression in the nucleus accumbens', J Neurosci, 24: 6939-45.

360. Clarke, R. B., and L. Adermark. 2010. 'Acute ethanol treatment prevents endocannabinoid-mediated long-lasting disinhibition of striatal output', Neuropharmacology, 58: 799-805.

361. Adermark, L., S. Jonsson, M. Ericson, and B. Soderpalm. 2011. 'Intermittent ethanol consumption depresses endocannabinoid-signaling in the dorsolateral striatum of rat', Neuropharmacology, 61: 1160-5.

362. Guegan, T., J. P. Cebria, R. Maldonado, and M. Martin. 2016. 'Morphine-induced locomotor sensitization produces structural plasticity in the mesocorticolimbic system dependent on CB1-R activity', Addict Biol, 21: 1113-26.

363. Zhao, X., et al. 2017. 'Cannabinoid 1 receptor blockade in the dorsal hippocampus prevents the reinstatement but not acquisition of morphine-induced conditioned place preference in rats', Neuroreport, 28: 565-70.

364. Hryhorowicz, S., M. Walczak, O. Zakerska-Banaszak, R. Slomski, and M. Skrzypczak-Zielinska. 2018. 'Pharmacogenetics of Cannabinoids', Eur J Drug Metab Pharmacokinet, 43: 1-12.

365. Sachse-Seeboth, C. et al. 2009. 'Interindividual variation in the pharmacokinetics of Delta9-tetrahydrocannabinol as related to genetic polymorphisms in CYP2C9', Clin Pharmacol Ther, 85: 273-6. 


\section{VITA}

Cory Lee Parks was born in 1991 to Alva Ray Parks and Teresa Louise Parks in West Plains, MO. In 2014, he earned a Bachelor of Science degree in Biomedical Sciences from Southeast Missouri State University. After graduating Cory spent two years working as a research technician in Dr. Salvatore Mancarella's lab in the Department of Physiology at the University of Tennessee Health Science Center where he matriculated into the Integrated Program of Biomedical Sciences the Fall of 2016. Under the guidance of Dr. Megan Mulligan in the Department of Genetics, Genomics, and Bioinformatics Cory completed his PhD candidacy in June of 2019 and defended his dissertation a year later in July of 2020. He expects to graduate with his doctorate in Biomedical Sciences in November 2020. 